\title{
Piezoelectric Transformer Plasma \\ Ion Source Characterization for \\ Neutron Production
}

\author{
A Thesis \\ presented to \\ the Faculty of the Graduate School \\ University of Missouri-Columbia
}

In Partial Fulfillment

of the Requirements for the Degree

of Doctor of Philosophy

by

Emily A. Baxter

Dr. John Gahl, Thesis Supervisor

May 2017 
The undersigned, appointed by the Dean of the Graduate School, have examined the thesis entitled:

\section{Piezoelectric Transformer Plasma \\ Ion Source Characterization for Neutron Production}

presented by Emily A. Baxter

a candidate for the degree of Doctor of Philosophy

and hereby certify that in their opinion it is worthy of acceptance.

\begin{tabular}{c}
\hline Dr. John Gahl \\
\hline Dr. Greg Dale \\
\hline Dr. Chi Ren Shyu \\
\hline Dr. Naz Islam
\end{tabular}




\section{Acknowledgements}

I would like to thank Dr. Gahl for coming into an impossible situation, and making it better. I would like to thank Drs. Dale, Shyu, and Islam for your participation in this endeavor.

I would like to thank Dr. Trauth, Dr. Gahl, Dr. Shyu, Terrance Grus, Dr. Rubin, Dean Loboa, Salama Gallimore, and the many others who helped me through a difficult (and sometimes seemingly impossible) situiation. Without all of you, this would never have happened.

Thank you to Drs. Benwell, Hutsel, Norgard, and Van Gordon, Major Olsen, Abe Dirnberger, and the many other former coworkers that helped me to get through this. Without Andy I would never have started this, and without the rest of you, I never would have finished.

Thank you to Kari Hamrick and Scott Dolezal for being my editors. Thank you to Kari and Larry Warner for your support in other matters. Thank you both for your constant support. Your help has been immeasurable and immense.

Thank you to the Kansas City Baxter family and the DeFily family for always being there for me. Your support means more than you will know.

Marcus Petree, your friendship through all this (the good times and the bad) has been so benefitial. I cannot thank you enough.

Finally, to my parents, words cannot express how much your support has meant to me. You have been there for all of my half-baked career paths (from dreams of tobacco lobbyist to concert pianist to rocket scientest) and you have been all in for all of them. I hope that you know how much you mean to me, and I can only hope to have made you proud.

To all those that I have not mentioned, thank you for helping me to become the crazy, intense, outspoken, feminist, scientest that I have become. Without all of you, I would not be where I am today. 
"Life is not easy for any of us. But what of that? We must have perseverance and above all confidence in ourselves. We must believe taht we are gifted for something and that this thing must be attained." - Marie Curie 


\section{Contents}

$\begin{array}{ll}\text { Acknowledgements } & \text { ii }\end{array}$

List Of Figures vii

1 Introduction $\quad 1$

1.1 Radioisotope Neutron Generators ... . . . . . . . . . . 2

1.2 Electronic Neutron Generators . . . . . . . . . . . . . 3

1.2.1 Electronic Neutron Generator Targets . . . . . . . . . 4

1.2.2 Electronic Neutron Generator Accelerating Voltage Sources 5

1.2.3 Electronic Neutron Generator Ion Sources . . . . . . . 5

1.3 Piezoelectric Transformer Plasma Source (PTPS) . . . . . . . . . 10

2 Theory 11

2.1 Ion Current Model . . . . . . . . . . . . . . . . . . . . 11

2.2 Electrostatic Probe Theory . . . . . . . . . . . . . 14

2.2.1 Plasma Sheath . . . . . . . . . . . 14

2.2.2 Types of Electrostatic Probes . . . . . . . . . . 16

2.2.2.1 Single Electrostatic Probes . . . . . . . . 18

2.2.2.2 Double Electrostatic Probes . . . . . . . 22

2.2.2.3 Triple Electrostatic Probes . . . . . . . . 24

2.2.3 Ion Trajectories in the Saturation Region . . . . . . . 26

2.2.4 Theories for Probe Current Correction . . . . . . . . 29 
2.2.5 Error in Electrostatic Probe Measurements . . . . . . . . 31

2.3 Hydrogen Ion Concentration . . . . . . . . . . . . . 34

3 Experimental Set-Up 36

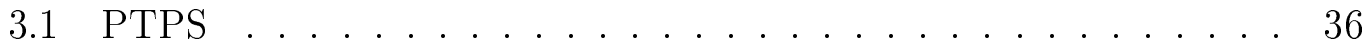

3.2 Ion Source Characterization Experiment . . . . . . . . 38

3.3 Neutron Experiment . . . . . . . . . . . . . 43

3.4 Triple Langmuir Probe Experiment . . . . . . . . . . . . . 44

4 Experimental Results $\quad 52$

4.1 Ion Source Characterization . . . . . . . . . . . 53

4.1.1 Deuterium and Hydrogen Gas Testing . . . . . . . . 53

4.1.2 Extraction Voltage Testing ........... 54

4.1.3 Gap Distance Testing . . . . . . . . . . . 56

4.1.4 RF Voltage Testing . . . . . . . . . . . . 58

4.1.5 Hollow Anode Radius Testing . . . . . . . . . . . . 59

4.1.6 Internal Pressure Testing . . . . . . . . . . . . 59

4.1.7 Holder Depth Testing . . . . . . . . . . . . . 64

4.1.8 Operational Time Testing ............ 65

4.1 .9 Ion Optic Simulation . . . . . . . . . . . 66

4.1.10 Summary of Ion Source Characterization . . . . . . . . 67

4.2 Neutron Generation Testing . . . . . . . . . . . . 69

4.3 Electron Temperature and Density Estimates . . . . . . . . 70

4.4 Ion Current Model . . . . . . . . . . . . . . . 77

5 Conclusions $\quad 84$

5.1 Ion Source Characterization Experiment . . . . . . . . 84

5.2 Neutron Generation Experiment . . . . . . . . . . . 87

5.3 Electrostatic Probe Experiment . . . . . . . . . . 87

5.4 Ion Current Model . . . . . . . . . . . . . . . . . 88 
$5.5 \quad$ Summary $\ldots \ldots \ldots \ldots \ldots \ldots \ldots$

$\begin{array}{ll}\text { Bibliography } & 89\end{array}$

$\begin{array}{ll}\text { VITA } & 106\end{array}$ 


\section{List of Figures}

Figure

page

2.1 Sheath model. . . . . . . . . . . . . . . . . 16

2.2 Sheath configurations. . . . . . . . . . . . . . 17

2.3 Single probe circuit. . . . . . . . . . . . . . . 18

2.4 Ideal current-voltage characteristic for an electrostatic probe. . . . 19

2.5 Double probe circuit. . . . . . . . . . . . . . . . 23

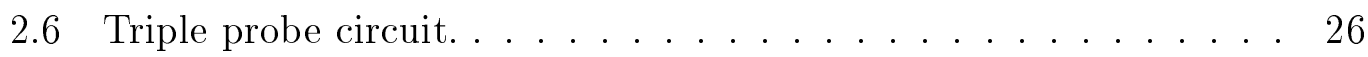

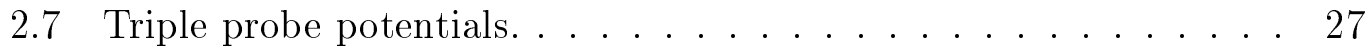

2.8 The ion trajectories when the probe is biased in the ion saturation

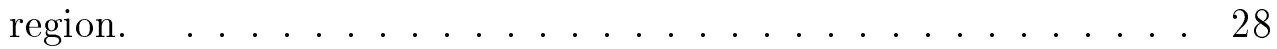

3.1 Cross-section of the PTPS. . . . . . . . . . . . . 37

3.2 Converse and direct piezoelectric effect. . . . . . . . . 38

3.3 Cross section and ion current diagnostic of the PTPS. . . . . . 40

3.4 Electric field profile of the extraction field of the PTPS ion source characterization experiment. . . . . . . . . . . . 41

3.5 Schematic diagram of the PTPS-DC neutron generation system. 45

3.6 X-rays measured at auto-transformer stepping ratios ranging from 50 to $90 \%$ of the total dc voltage supply. . . . . . . . . . 45

3.7 Schematic diagram of the triple probe diagnostic for the PTPS. . 46 
3.8 Cross section of the triple probe system used for electron temperature and density measurements. . . . . . . . . . . 51

3.9 Triple probe voltage and current data compared to input voltage. 51

4.1 Measured hydrogen and deuterium ion current. . . . . . . . . . 54

4.2 Measured current for a range of RF driving voltages and extraction

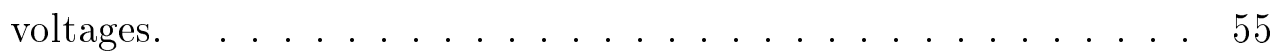

4.3 Measured current versus perveance for two internal pressures. . . 56

4.4 Measured current versus perveance for three gap distances. . . . 57

4.5 Measured current for a range of RF driving voltages and extraction

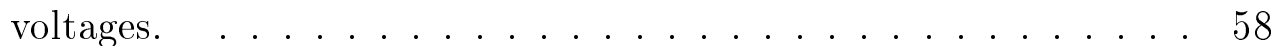

4.6 Measured ion current for a range of pressures with 0.15 and $0.3 \mathrm{~mm}$ hollow anode radii. ................ 60

4.7 Measured ion current for a range of pressures from 1.5 to 11.0 Torr. 63

4.8 Averaged measured ion current for a range of pressures from 1.5 to 11.0 Torr. . . . . . . . . . . . . . . . 64

4.9 Measured ion current for a holders with a 3, 6, and $9 \mathrm{~mm}$ depth. $\quad 65$

4.10 Normalized measured ion current over time. . . . . . . . . . . . 66

4.11 COMSOL simulation of meniscus formed inside the hollow anode aperture at $2000 \mathrm{~V}$ extraction. . . . . . . . . . 67

4.12 COMSOL simulation of the PTPS with a $2.4 \mathrm{~mm}$ opening optic at $-100 \mathrm{~V}$ at different spacing from the $0.3 \mathrm{~mm}$ radius hollow anode with the $-1000 \mathrm{~V}$ extraction voltage. . . . . . . . . 68

4.13 Neutron counts as a function of accelerating potential. . . . . . 71

4.14 Measured electron temperature for a range of extraction voltages from -700 to $-2000 \mathrm{~V}$. . . . . . . . . . . . 73 
4.15 Measured ion current over a range of dc extraction voltages from 700 to $2000 \mathrm{~V}$ with a $0.3 \mathrm{~mm}$ hollow anode radius, an RF input voltage of $350 \mathrm{~V}$, and an internal pressure of 1.25 Torr plotted with ion current calculated using (2.1) for $\mathrm{H}_{3}{ }^{+}$ions at an electron temperature of $4.45 \mathrm{eV}$ and density of $2.5 \times 10^{15} \mathrm{~m}^{-3}$. . . . . 74

4.16 Measured electron densities for a range of extraction voltages from

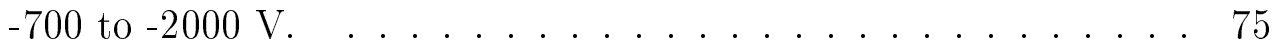

4.17 Calculated electron densities for a range of extraction voltages from -700 to $-2000 \mathrm{~V}$. . . . . . . . . . . . 76

4.18 Measured ion current for a range of RF input voltages from -175 to $-350 \mathrm{~V}$ and internal pressures from 0.75 to 1.25 Torr. . . . . . . 77

4.19 Measured electron temperature for a range of RF input voltages from -175 to $-350 \mathrm{~V}$ and internal pressures from 0.75 to 1.25 Torr. 78

4.20 Measured electron density using $\mathrm{H}_{3}{ }^{+}$ions for a range of $\mathrm{RF}$ input voltages from -175 to $-350 \mathrm{~V}$ and internal pressures from 0.75 to 1.25 Torr. . . . . . . . . . . . . . . 78

4.21 Measured hydrogen ion current and modeled ion current using a large range of particle densities. . . . . . . . . 80

4.22 Measured hydrogen ion current and modeled ion current using a small range of particle densities. . . . . . . . . . . 81

4.23 Measured hydrogen ion current and modeled ion current using a range of electron temperatures. . . . . . . . . . . 82

4.24 Measured and predicted hydrogen current. . . . . . . . . . . 83 


\section{Chapter 1}

\section{Introduction}

Neutron sources have a variety of applications because of the ability of neutrons to non-destructively determine the elemental composition of a material $[1,2]$. Neutron sources are used for national security purposes through neutron active interrogation of materials [1-10]. In neutron active interrogation, neutrons enter the nuclei of a sample, producing a measurable reaction, allowing for the determination of the target material [1,3-11]. For example, in explosives detection, neutrons are used to determine the elemental signature of a material, which can then be compared to the signature of most explosives [5, 12]. Industrial quality control uses neutrons to characterize properties of new materials, and for rapid analysis of unknown materials [13, 14]. Neutron sources are used in geophysical exploration for soil monitoring to determine the concentration of water in the soil for construction and agriculture [2-4]. One of the most used applications for neutron sources is oil reserve detection in boreholes for oil well logging $[3,4,6,15,16]$.

According to the U.S. Energy Information Administration, more than 19.1 million barrels of oil were consumed each day in the United States in 2014; the United States only produced about 8.7 million barrels per day. Large demands for oil will continue to drive the need for an increase in the number and performance of oil wells. Oil reserves are typically located and cataloged through 
oil well logging which is a method of characterizing the porosity and density of a borehole to determine the quantity of hydrocarbons remaining and the location of these hydrocarbons for oil production [6, 16-20].

Two methods common to the logging of oil wells are coring and neutron logging [17]. Coring involves physically removing a sample from the sidewall of the borehole, and analyzing it at the surface [17]. This time consuming method only provides information on a small sample of the borehole. Neutron logging, however, analyzes a large portion of the borehole using a neutron source and detector which are lowered into the borehole [6, 16-20]. Neutrons probe the sidewall using the $(n, \gamma)$ reaction. The $(n, \gamma)$ reaction is measured by a detector and used to determine the elemental makeup of the sidewall. A signal is sent back to an operator through a wire line attached to the radiation detector and a $\log$ is kept of the density of the borehole at different depths. The porosity of the borehole is measured by the ratio of the count rates from near and far neutron detectors which allow for a measure of the neutron penetration into the formation [17, 19]. Most current methods of oil well logging use radioisotope neutron generators $[5,12,21]$.

\subsection{Radioisotope Neutron Generators}

Radioisotope neutron generators, including americium-beryllium (Am-Be) and plutonium-beryllium $(\mathrm{Pu}-\mathrm{Be})$ sources, are traditionally used in oil well logging [5, 12, 21]. In radioisotope neutron sources, neutrons are produced through the $(\alpha, n)$ reaction where alpha particles are emitted by an alpha emitter, such as ${ }^{241} \mathrm{Am}$ or ${ }^{239} \mathrm{Pu}$, and captured by ${ }^{9} \mathrm{Be}$ which in turn releases a neutron [17, 19]. Approximately one neutron is emitted for every 20,000 alphas [22]. Radioisotope sources remain active for years, but are unable to be turned off, thus they require radiation shielding and special considerations for transport as the activity of these sources can range from 5 to $20 \mathrm{Ci}[3,19,20]$. These 
sources also become a security risk if stolen, or an environmental risk if lost in a borehole [20, 22]. Alternative neutron sources, including electronic neutron sources, can be used as a replacement for radioisotope sources, reducing the risks associated with the radioactive sources.

\subsection{Electronic Neutron Generators}

Electronic neutron generators produce neutrons through hydrogen isotope fusion. These sources are either sealed tube- or pumped neutron generators. Electronic neutron generators have three main components: a target, an accelerating voltage source, and an ion source [1,5]. Sealed tube neutron generators tend to be more compact, portable, and efficient when compared to pumped generators, but require a pressure regulating device because there is no external gas supply and a system to replenish the target for prolonged use [1, 2]. While pumped systems are known for higher neutron yields, they are generally stationary due to their size and the need for continuously pumped gas [1].

Electronic neutron generator systems use the deuterium-deuterium (D-D) or deuterium-tritium (D-T) reaction to generate neutrons as shown in (1.1) and (1.2), respectfully [5, 23]. Deuterium ion beams are ideal for neutron generators because of the low binding energy between the single neutron and proton and require a low voltage (on the order of $10 \mathrm{kV}$ ) to initiate the reaction [1]. Depending on the desired neutron energy, a beam of deuterium ions is accelerated toward a target containing either deuterium or tritium. The fusion reaction of the ion beam with the target generates either a 2.45 MeV neutron for D-D fusion or a $14 \mathrm{MeV}$ neutron for D-T fusion [1, 2, 11, 13].

$$
\begin{aligned}
& { }_{1}^{2} \mathrm{D}+{ }_{1}^{2} \mathrm{D} \rightarrow{ }_{2}^{3} \mathrm{He}+n \\
& { }_{1}^{2} \mathrm{D}+{ }_{1}^{3} \mathrm{~T} \rightarrow{ }_{2}^{4} \mathrm{He}+n
\end{aligned}
$$


Electronic neutron generators using hydrogen fusion reactions have advantages and disadvantages. Some advantages of D-D electronic neutron generators include the ability to be turned off and to produce nuclear reactions without the use of radioisotopes, however improvements are necessary to produce electronic sources that are more compact and efficient $[3,19]$. While the D-T reaction generates a higher energy neutron at at higher yield due to the larger reaction cross section, a radioactive component (tritium) is also present which requires additional safety precautions over D-D sources because of the health hazards related to ingestion or inhalation $[2,10,11]$.

Most neutron sources have a yield on the order of $10^{8} \mathrm{n} / \mathrm{s}$ or higher. The neutron yield of the source can be increased through increasing either the ion beam current impinging on the target, the loading of the target, or the ion accelerating voltage $[1,2,11]$. The number of ions reaching the target can be improved through ion beam collimation using ion beam optics or tubing $[1,2,24]$. The neutron yields of these sources are limited by the breakdown of high voltage across the accelerating gap, ion source output, and the ability of the target to withstand power loading and capacity of the target for hydrogen loading [1, 11].

\subsubsection{Electronic Neutron Generator Targets}

Targets are chosen for their ability to withstand high power inputs and their capacity for hydrogen loading. Electronic neutron generator targets require a long lifetime to tolerate a constant neutron flux over long periods of time because target removal is difficult and can result in significant downtime [1]. Targets should be capable of high neutron yields per unit area meaning they should be saturated with deuterium or tritium ions over a small space [1].

Sealed tube neutron generators typically use solid targets. Solid targets are a metal substrate either doped with a hydrogen isotope, or with a thin film attached

which provides good conductivity and is doped with the hydrogen isotope $[1,2$, 
11]. Metals used for hydrogen impregnated sources include titanium, zirconium, and molybdenum [1, 2, 11]. Solid targets are characterized as being stable with high thermal conductivity and have the ability to endure the application of large beam currents, all of which could cause drastic degradation of performance in other types of targets [1]. Some neutron generators employ various target cooling methods to prevent hydrogen dissociation when the critical temperature of the solid target is met $[1,2]$.

\subsubsection{Electronic Neutron Generator Accelerating Voltage Sources}

Accelerating voltage has direct effects on the neutron yield of an electronic neutron generator, thus it should be at its maximum value without causing breakdown in the accelerating gap $[1,2,11]$. A good voltage source for neutron generators is easily regulated resulting in low voltage fluctuations, a low temperature coefficient reducing ion drift problems, and a fast recovery time if the load is changed [1]. Two voltage accelerator designs common to neutron generators are electrostatic sources such as Van de Graff or Felici and the Cockroft-Walton voltage multiplying design [1]. Electrostatic design characteristics include minimal voltage fluctuation, excellent voltage regulation, and quick response to load changes, however the electrostatic designs are typically larger and more expensive than other designs [1]. The Cockroft-Walton generators rarely fail, are typically inefficient and difficult to scale the power [1].

\subsubsection{Electronic Neutron Generator Ion Sources}

Sealed tube neutron generators require a source of ions [1, 5]. Ion beam sources generally consist of an ion reserve (where ionization occurs) and an extraction system for focusing and accelerating the ion beam [25-27]. The simplest ion source design consists of a plasma source and an extraction method 
used to accelerate beams of ions from the plasma to a target [1, 25, 28, 29]. The plasma source uses a high voltage to ionize a gas, resulting in a quasineutral plasma containing nearly equal numbers of ions and electrons [28, 30]. In an equilibrium plasma, the generation rate of ions is equivalent to the loss rate, including losses through ion extraction and ion-electron recombination [2528, 31]. The ion current extracted from the plasma is limited by the Bohm sheath criterion through plasma saturation and the Child-Langmuir law through the space-charge limit [26, 31].

Plasma ion sources consist of gas and high frequency discharges. In gas discharges, a voltage is applied between two cathodes immersed in a gas [26]. As the voltage on the cathodes or the pressure in the chamber increases a discharge occurs [26]. A de discharge can be characterized as a Townsend, arc, or glow discharge depending on the gas pressure in the ionization chamber [26]. High frequency discharges occur at frequencies greater than $100 \mathrm{kHz}$ when the inertia of the ions prevents regrouping [26]. High frequency systems are generally either radio frequency or microwave plasma ion sources [26]. Three plasma ion sources commonly used in neutron generators include the Penning-, radio frequency-, and electron cyclotron resonance ion sources [1, 2, 25-27].

The Penning ion source is a dc glow discharge source that can be operated with hot or cold cathodes [1, 25-27, 29, 32-37]. There are four main components in Penning ion souces: two cathodes, an anode, and a permanent magnet. In the Penning design, electrons are released from the cathodes toward an annular anode located around the cathodes [1, 25-27, 29, 32-37]. Further collisions of the oscillating electrons with gas molecules generates an intense plasma in the ionization chamber [26]. The permanent magnet is used to apply an axial magnetic field that restricts angular motion of the electrons, increasing the ionization efficiency resulting in approximately eight ions per electron [26, 29].

Penning ion sources can produce ion currents in the micro- to milli-amp range, 
but the current should be limited to avoid damage to the ion source [1, 2527, 29, 32-37]. In order to maintain stable currents, the Penning ion source has an optimal operating range. Operating at too high of a current can cause anode heating resulting in gases being released from the metal; this situation results in uncontrollable pressures in the neutron generator and unpredictable results [33]. Penning ion sources can be operated in either pulsed or dc mode, but have a low atomic-to-molecular ion ratio, with greater than $90 \%$ molecular ions in some cases [1, 2, 37]. This leads to decreased neutron yield because the acceleration energy is distributed over the two nucleons in diatomic ions [1, 2, 37]. Metal sputtering is also a common problem in Penning ion sources because the housing is made entirely of metal [1,29]. Sputtering results in the need for the ion source to be cleaned periodically and, occasionally, the cathodes and anode must be replaced [1, 29]. Penning ion sources are capable of ion currents in the $100 \mu \mathrm{A}$ range with operating times greater than 500 hours and have been integrated into compact portable neutron generator systems capable of D-D neutron yields of at least $10^{8} \mathrm{n} / \mathrm{s}[1,25-27,29,32-37]$.

The radio frequency, or RF, ion source is a high frequency discharge ion source which generally operates in the $1-100 \mathrm{MHz}$ range $[1,26]$. The electrons in the gas oscillate due to the RF field, and eventually gain enough energy to generate a plasma by ionizing the gas [1, 29]. In RF ion sources, a gas is introduced into an ionization chamber at a controlled rate and is ionized by the RF voltage using an electrode system that is not submerged in the plasma [1]. The ionization chamber is generally fabricated out of a dielectric such as glass or quartz to prevent sputtering and limit recombination and secondary electron emission [1]. The RF ion source typically uses either a capacitive or inductive method of supplying the RF voltage to the ionization chamber to excite the gas [29]. A magnetic field can be added to the RF ion source to focus the ion beam [1].

There are many reasons the RF ion source is attractive for neutron generators. 
These sources have shown ion beam currents ranging from $70 \mu \mathrm{A}$ to greater than $5 \mathrm{~mA}$, with the currents being modulated with: ionization chamber pressure, RF input voltage, external magnetic field, and extraction voltage allowing for scalable operation [11, 13, 29, 38-42]. However, these sources have a low gas efficiency, generally requiring vacuum pumping to keep the ion source at reasonable operating pressures making them comparatively less portable, but can produce an atom-to-molecule ratio of 9 [1, 2]. RF ion sources have been shown to produce $80-95 \%$ atomic species fraction, which results in efficient neutron production on the order of $10^{8} \mathrm{n} / \mathrm{s}$ for the D-D reaction and $10^{11} \mathrm{n} / \mathrm{s}$ for the D-T reaction [11, 38-41]. Additionally, RF ion sources are compact and relatively portable with long operational lifetime (on the order of hundreds of hours). A disadvantage of RF ion sources include sputtering of any metal in the system by the plasma resulting in metallic coating on the dielectric walls causing recombination and lowered efficiency $[1,26,27,38]$. The ion source can be taken apart and cleaned, restoring the high output capability of the ion source, but this tends to be time consuming and can result in the contamination of the vacuum system [1].

The electron cyclotron resonance, or ECR, ion source is also a high frequency discharge which operates in the giga-Hertz range [26, 29]. Electron cyclotron resonance heating occurs in a plasma when the electrons are heated using a microwave coupled into the plasma at the electron cyclotron frequency [29]. In these sources, the microwave is used to generate the plasma and a magnetic field is used to trap the plasma, making the plasma more dense resulting in a high energy (up to $10 \mathrm{kV}$ ) non-Maxwellian plasma [29, 43]. Because of the low cost and wide availability of microwave sources at this frequency, these sources commonly operate at a frequency of $2.45 \mathrm{GHz}$ [29]. Additionally, at $2.45 \mathrm{GHz}$, the waveguide required to supply the microwave to the plasma remains small; the magnetic field required to confine the plasma is relatively small (less than 
$0.1 \mathrm{~T})$; and fewer low energy electrons are produced. This results in less multiply charged ions [29, 43].

The ECR ion source is capable of producing high currents of singly charged ions [26, 27, 29, 43-47]. There is no ignition process for ECR ion sources making them easy to operate and no electrodes allowing for operation with aggressive gases without concern for electrode damage or contamination [29, 45, 47]. Also the ECR operates at low pressures making voltage breakdown unlikely [45, 47]. The ECR has been operated in both pulsed and continuous mode with ion currents reaching $100 \mathrm{~mA}$ for operating times up to 2000 hours [26, 27, 43-47]. These sources are efficient, showing singly charged ion percentages ranging from $70-98 \%$, leading to neutron yields up to $10^{10} \mathrm{n} / \mathrm{s}$ and $10^{12} \mathrm{n} / \mathrm{s}$ for D-D and D-T fusion reactions, respectively [26, 27, 43-47]. The drawbacks to ECR ion sources include: high power operational requirements (up to $1 \mathrm{~kW}$ ), requirements for magnet cooling, and the high dependence on the source subsystems for stability (including the required impedance matching between the microwave circuit and plasma, the magnetic field strength and distribution in the plasma, as well as the operating pressure of the plasma) [29, 45, 47].

Piezoelectric transformers (PTs) can be used to generate the high voltages required for compact, low input voltage plasma formation which can be used in ion sources [48]. Fabricated from ferroelectric materials such as $\mathrm{Pb}(\mathrm{ZrTi}) \mathrm{O}_{3}(\mathrm{PZT})$ and $\mathrm{LiNbO}_{3}$ (lithium niobate) as high voltage is generated through electrical-mechanical energy conversion based on the piezoelectric effect [48-51]. A sinusoidal voltage is applied to the PT and through the converse piezoelectric effect causes mechanical deformation and simultaneously induces vibration in the $\mathrm{PT}[10,48,52-55]$. If operated at the natural vibrational resonance of the PT an electrical-mechanical resonance occurs [10, 48, 52-55]. A high voltage occurs through the mechanical vibration caused by the direct piezoelectric effect. By flowing gas into the area of high voltage a plasma can 
form [10, 48, 52-55].

Piezoelectric transformers have been studied as plasma sources with pressure ranging from $10^{-2}$ and $10^{3}$ Torr $[52,56-62]$. Three types of PT plasma sources that have been studied include: corona, dielectric barrier, and glow discharge plasma sources [48]. The corona discharge plasma source requires at least one sharp electrode be attached directly to the PT. At pressures near atmosphere corona appears around the electrode [63,64]. Dielectric barrier discharges are also operated at atmospheric pressures. A small discharge is formed in the slight gap between the metallic electrode and PT surface $[52,60,62]$. In a glow discharge the PT is operated in low background pressures [56-59].

\subsection{Piezoelectric Transformer Plasma Source (PTPS)}

The piezoelectric transformer plasma source (PTPS) was investigated as a compact, radioisotope free plasma ion source for use in an electronic neutron generator. The size of the PTPS is minimized because the ionizing voltage is produced through a radial mode piezoelectric transformer (PT) [65, 66]. Ion currents produced by the PTPS can be modulated through changes to the driving voltage, which allows for scaling of the source ion output. The PTPS can be operated as a source of deuterium ions to facilitate the D-D reaction. The PTPS could provide improvements to current ion source technologies for electronic neutron generators. 


\section{Chapter 2}

\section{Theory}

This chapter outlines three theories for reference in the remainder of the dissertation. The ion current model allows for the determination of the ion current that can be drawn through the hollow anode towards the extraction target based on the parameters of the plasma. The electrostatic probe theory section provides information about the background and operation of electrostatic probes as well as sources of and corrections for errors in probe measurements. Finally, the ion concentration of hydrogen plasmas is discussed, showing the different reactions possible in a hydrogen plasma similar to the one found in the piezoelectric transformer plasma source (PTPS).

\subsection{Ion Current Model}

The ion current extracted from the plasma is pulled from the sheath area over the hollow anode aperture [67-73]. This sheath, sometimes called the meniscus of the plasma, is partially caused by the applied electric field used to extract the ions from the plasma through the hollow anode [67-73]. At the meniscus edge the ions reach the Bohm velocity, and are released from the plasma [73]. The ion current of the plasma can be predicted using the Bohm current density multiplied by the extraction area. The concave meniscus provides the optimal 
ion beam focusing, though overfocusing can occur resulting in unwanted ion beam divergence $[69,70,72,74]$. While it may be impossible to generate the ideal concavity of the beam [68], the shape of the meniscus is controlled by the electron density and temperature of the plasma as well as the extraction voltage and hollow anode shape [69, 72, 74]. The ion current can be modeled using (2.1), with variables found in Table $2.1[69,73,74]$.

$$
\begin{gathered}
I_{m}=(0.61) \varepsilon_{o} \sqrt{\frac{e}{M}} \pi \alpha^{2} F T_{e}^{\frac{3}{2}} \\
\lambda_{D}=\sqrt{\frac{\varepsilon_{o} T_{e}}{e n_{e}}}
\end{gathered}
$$

Table 2.1: Variables and Units for (2.1)

\begin{tabular}{ccc}
\hline Variable & Description & Units \\
\hline \hline$I_{m}$ & modeled current & $\mathrm{A}$ \\
$\varepsilon_{0}$ & permittivity of free space & $\mathrm{F} / \mathrm{m}$ \\
$e$ & elementary charge & $\mathrm{C}$ \\
$M$ & ion mass & $\mathrm{kg}$ \\
$\alpha$ & constant of proportionality, $\alpha=\frac{a}{\lambda_{D}}$ & unitless \\
$F$ & function of electric field ratio, $F\left(E_{0} / E\right)$ & $\mathrm{unitless}$ \\
$E_{0}$ & extraction electric field & $\mathrm{V} / \mathrm{m}$ \\
$E$ & electric field characteristic of the plasma, $E=\frac{T_{e}}{\lambda_{D}}$ & $\mathrm{~V} / \mathrm{m}$ \\
$T_{e}$ & electron temperature & $\mathrm{eV}$ \\
$a$ & hollow anode radius & $\mathrm{m}$ \\
$\lambda_{D}$ & Debye length & $\mathrm{m}$ \\
$n_{e}$ & plasma electron density & $\mathrm{m}^{-3}$ \\
\hline
\end{tabular}

Several functional relationships can be made to determine methods of increasing ion currents based on (2.1) to direct experiments for ion source optimization. Based on (2.1) the parameters which have an impact on the ion current are: ion mass, hollow anode radius, plasma electron density, extraction electric field, and electron temperature. 
The ion mass affects the ion current by a factor of the inverse square root of the change in ion mass $(1 / \sqrt{\triangle M}$ where $\triangle M$ is the change in ion mass). For example, assuming the mass of deuterium ions are twice that of hydrogen ions, the functional relationship between the currents would be $I_{D} \sim I_{H} / \sqrt{2}$, where $I_{D}$ is the deuterium ion current and $I_{H}$ is the hydrogen ion current.

As stated previously, the hollow anode radius should have a significant impact on the ion current. The constant of proportionality, $\alpha$, has a strong dependence on the hollow anode radius. The hollow anode radius also impacts the internal pressure by partially controlling the rate at which gas escapes through the plasma chamber.

The electron density of the plasma affects the ion current through the Debye length. The Debye length is taken into account in (2.1) through the constant of proportionality, which is a ratio of the hollow anode radius to the Debye length. The model predicts a current proportional to the square root of the plasma electron density $\left(I \propto \sqrt{n_{e}}\right.$, where $I$ is the ion current and $n_{e}$ is the electron density of the plasma).

The extraction electric field is a function of the extraction voltage and gap distance between the extraction voltage source and the hollow anode of the plasma source. The extraction is accounted for in (2.1) in $F$, which is a function of the extraction field and the electric field characteristic of the plasma $\left(F\left(E_{0} / E\right)\right.$, where $E_{0}$ is the extraction electric field and $E$ is the electric field characteristic of the plasma). The functional relationship is nearly linear over a typical range of $E_{0} / E$.

According to (2.1), the electron temperature also impacts the ion current directly as well as through the Debye length, resulting in a direct relationship between the ion current and the electron temperature $\left(I \propto T_{e}\right.$, where $I$ is the ion current and $T_{e}$ is the electron temperature of the plasma). 


\section{$2.2 \quad$ Electrostatic Probe Theory}

Electrostatic probes are commonly used to measure electron temperature and density of a plasma because they are easy to manufacture, have a large dynamic range, and provide local plasma measurements [75, 76]. In electrostatic probe systems at least one conductive object is inserted directly into the plasma; current and voltage diagnostics of the probe output are used to determine the characteristics of the plasma [75? -140]. Electrostatic probes tend to be low in cost and simple in operation while allowing for a local measurement over a large volume of the plasma, but can require complex analysis [30, 141, 142]. The probe can also cause unwanted affects on the plasma resulting in a change in the plasma characteristics attempting to be measured.

\subsubsection{Plasma Sheath}

The applied bias voltage on the probe causes a region of disturbance, known as the probe sheath, in which particles of the opposite charge collect around the probe as is shown in Fig. 2.1 for ions [31, 84, 85, 122, 133, 141, 143, 144]. While the plasma is assumed to be quasi-neutral (having equal numbers of ions and electrons), inside the sheath the plasma is not quasi-neutral and is composed of primarily one particle based on the potential of the probe [31, 85, 91, 143]. A negatively biased probe has an ion sheath, while a positively biased probe has an electron sheath. While the composition within the sheath is not the same as the bulk of the quasi-neutral plasma, it is possible to use the characteristics of the sheath to determine the characteristics of the plasma as a whole.

The size of the sheath calculated using (2.3) is a function of the Debye length and the ratio of the electron and ion masses [145]. The Debye length of the plasma $(H)$, described by $(2.2)$, is a function of the electron temperature and density of the plasma and describes the length from which a particle can screen the electrostatic fields of a particle of opposite charge [133]. 


$$
H=\frac{2}{3} \lambda_{D}\left(\frac{2}{\exp (-1)}\right)^{-\frac{1}{4}}\left(-\frac{1}{2} \ln \left(\frac{m_{e}}{m_{i}}\right)-\frac{1}{\sqrt{2}}\right)^{\frac{1}{2}}\left(\left(-\frac{1}{2} \ln \left(\frac{m_{e}}{m_{i}}\right)\right)^{\frac{1}{2}}+\sqrt{2}\right)
$$

Any collisions within the sheath are a function of the sheath and the mean free path of the ions in the plasma. The ion mean free path of a plasma is the distance an ion can travel before coming in contact with another particle resulting in additional ionization and loss of momentum [91]. This distance is found using (2.4), where $k_{B}$ is the Boltzmann constant, $T$ is the temperature of the gas (typically assumed to be $300 \mathrm{~K}$ ), $d$ is the molecular diameter of the gas $[\mathrm{m}]$, and $P$ is the internal gas pressure $[\mathrm{Pa}]$. The ion mean free path of the plasma is dependent on the pressure of the plasma; low pressure plasmas have long mean free paths and high pressure continuum plasmas have short mean free paths.

$$
\lambda_{i}=\frac{k_{B} T}{\sqrt{2} \pi d^{2} P}
$$

The operating regime for a probe in a non-magnetic plasma is commonly described by the Knudsen and Debye numbers [135, 136, 146]. The Knudsen number $\left(K_{i}\right.$ for ions) is the unitless ratio of the mean free path and the probe radius $\left(r_{P}\right)\left(K_{i}=\lambda_{i} / r_{P}\right)$. The Debye number $\left(D_{\lambda}\right)$ is the unitless ratio of the Debye length to the probe radius $\left(D_{\lambda}=\lambda_{D} / r_{P}\right)$.

There are two characteristics of the plasma sheath: collisionality and size [30, 31, 122]. A collisionless sheath has no collisions because the mean free path is larger than the sheath thickness, resulting in a collision free region. A collisional sheath is characterized by a sheath that is larger than the mean free path, resulting in collisions within the sheath. A transitional sheath is on the same order of magnitude as the mean free path. The sheath size (thick or thin) relates to the sheath proximity to the probe. The sheath 
characteristics are paired as shown in Fig. 2.2. The collisionless thin sheath shown in Fig. 2.2a and collisionless thick sheath shown in Fig. 2.2b represent the more ideal situations for determination of plasma characteristics. As shown in Fig. 2.2a and Fig. 2.2b, the mean free path is longer than the sheath, resulting in the particle theoretically taking a straight path toward the probe. In the collisional thin sheath shown in Fig. 2.2c and the collisional thick sheath shown in Fig. 2.2d, the particle takes a less direct path toward the probe. This less direct ion trajectory can result in errors. These errors can be corrected, but require the use of the appropriate theory to determine the most accurate plasma characteristics [76, 82, 86, 88, 96, 101, 103, 111, 113, 135, 136, 144, 147-149].

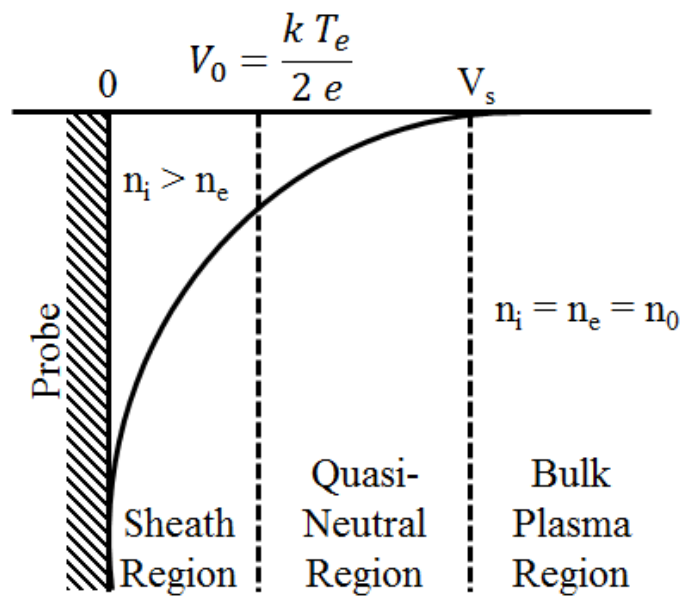

Figure 2.1: Sheath model with the probe at a negative potential in the ion collection regime.

\subsubsection{Types of Electrostatic Probes}

Three different types of electrostatic probe systems used to estimate the electron temperature and density of the plasma are single $[75,79,80,86$, 87, 94，96，100，103，105，112-114，117，119，126，128-132，135，136，150153], double [92, 103, 109, 110, 118, 125, 133, 154, 155], and triple [84, 89, 


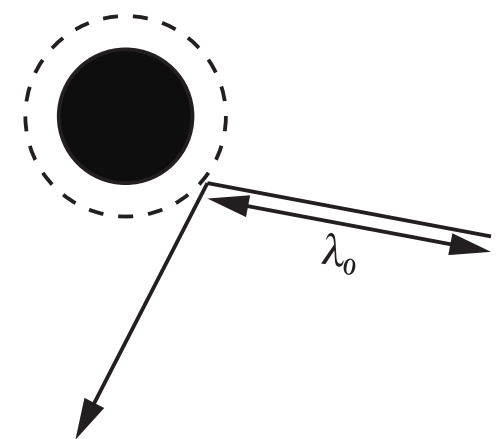

(a) Collisionless thin sheath

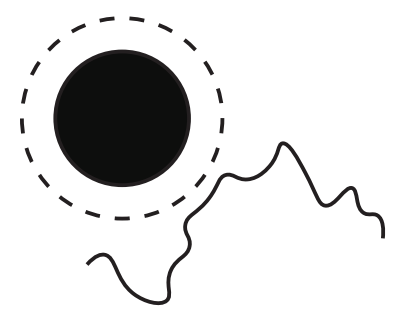

(c)

Collisional thin sheath

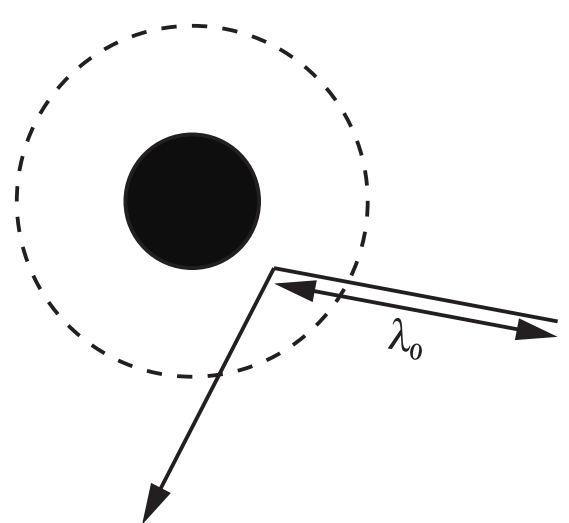

(b) Collisionless thick sheath

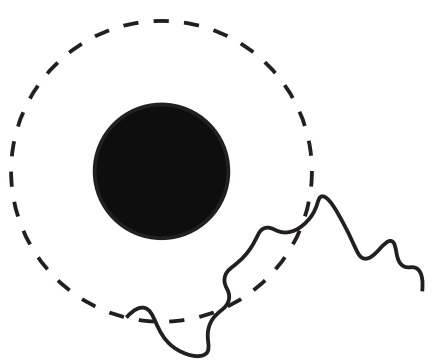

(d) Collisional thick sheath

Figure 2.2: Sheath configurations for collisionless and collisional thin and thick sheaths (dashed line) around the electrostatic probe (solid circle) with the approaching particle where $\lambda_{0}$ is the mean free path.

92, 102, 106, 107, 109-111, 119, 137, 147, 156] probe systems, corresponding to the number of probes in the system. These three systems have been shown to work in the collisional [76, 111, 113, 127, 135, 144, 147, 149], collisionless [86, 95, 102, 104, 109, 113, 125, 128-130, 132, 148, 150, 155, 157], and transition regimes $[75,86,103,125,135,136,148,153]$. Electrostatic probe systems have been compared with other plasma measurements to confirm their accuracy $[110,114,119,121]$. Probes are constructed of conductive metals that can withstand high heat loads including molybdenum, tungsten, platinum, and gold [91]. The shape of the probe can be planar, cylindrical, or spherical, though 
cylindrical probes are the simplest to make as they can be easily produced from a wire [90, 91, 141]. All discussions of probes in this section will assume a cylindrical geometry unless otherwise stated.

\subsubsection{Single Electrostatic Probes}

In single electrostatic probe experiments, the probe is inserted into the plasma, and the bias voltage to a probe is swept from a voltage negative of the plasma potential to a voltage positive of the plasma potential $[30,31,85$, 141, 142, 158]. Depending on the plasma being measured, this sweep can range from a few volts to hundreds of volts [30, 31, 85, 133, 141, 142, 158]. A simple single probe schematic is shown in Fig. 2.3 where $I$ is the current collected by the probe (typically measured using a current viewing resistor, and a differential probe) and $V_{B}$ is the applied bias voltage. During the voltage sweep, the current collected by the probe is monitored, then plotted as a function of the swept bias voltage. An ideal current-voltage plot is shown in Fig. 2.4. The plot reveals three distinct regions: ion saturation, transition, and electron saturation.

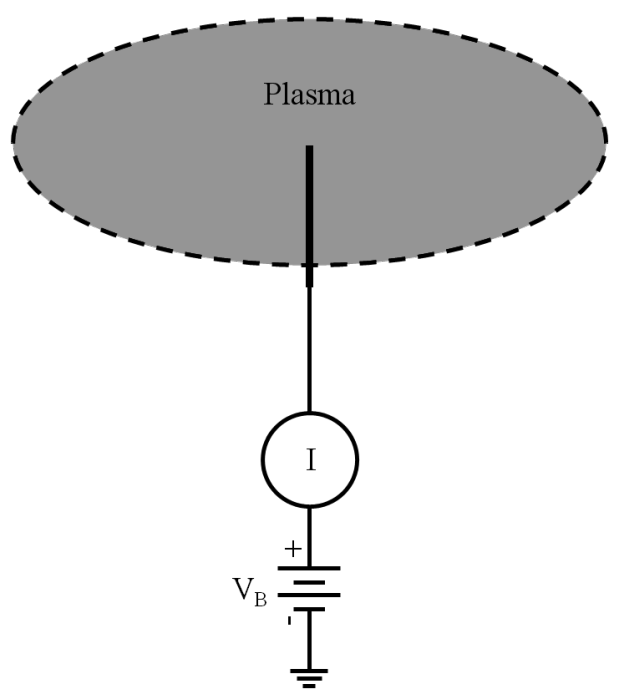

Figure 2.3: Schematic of a single electrostatic probe with a simple passive measurement circuit. 


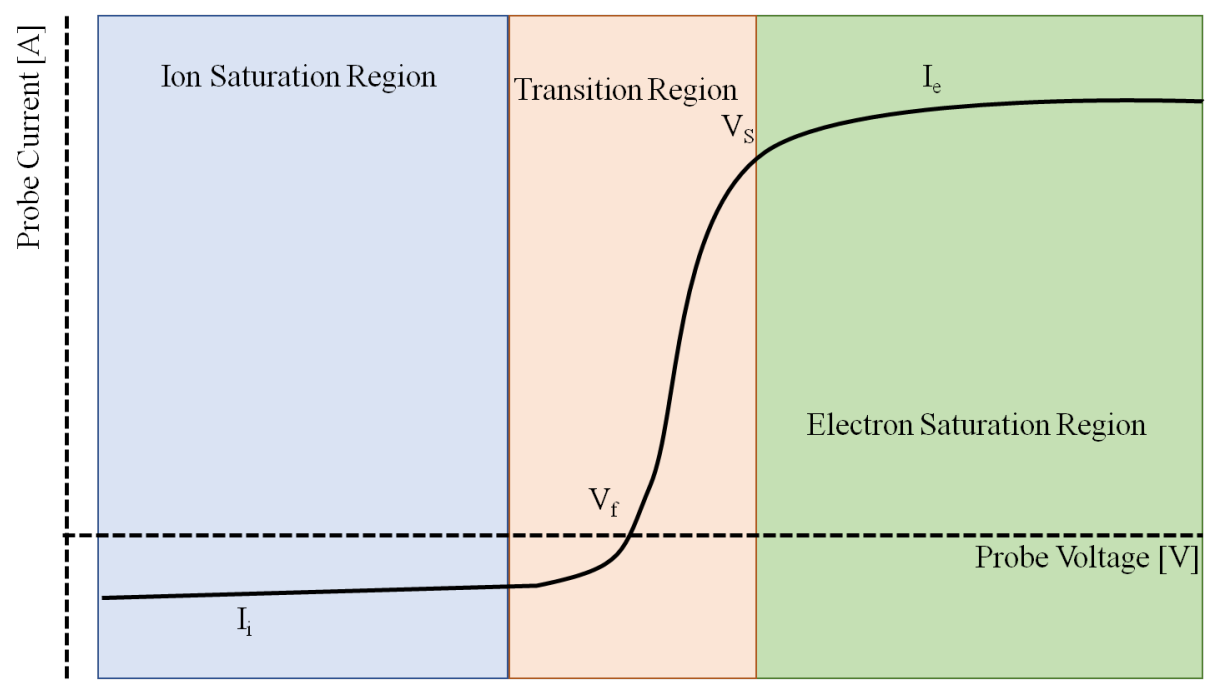

Figure 2.4: Ideal current-voltage characteristic for an electrostatic probe.

The first region is the ion saturation region where the probe bias voltage is negative with respect to the plasma (depicted as the left region in Fig. 2.4) [30, $31,85,91,133,141,145,158]$. In this region the sheath is small in comparison to other regions, and only ions reach the probe for measurement. In the ideal case, this region would not change with the probe bias voltage [30, 31, 85, 91, 133, $141,145,158]$. In reality, the sheath size increases with increasingly negative bias voltages $[30,31,85,91,133,141,145,158]$. This results in the ion saturation current decreasing as well $[30,31,127,158]$. This can be corrected by determining the slope equation for the ion saturation current, and then subtracting that slope from the measured current $[30,158]$. The ion saturation region is typically used to determine the electron density of the plasma through the measurement of the ion saturation current $\left(I_{i}\right)[30,31,85,91,133,141,145,158]$.

The second region of the current-voltage plot is the transition region (depicted as the center region in Fig. 2.4) [30, 31, 85, 91, 133, 141, 145, 158]. This region is characterized by exponential current growth with increasing bias voltage as an increasing number of electrons reaches the probe for measurement $[30,31,85$, 
91, 133, 141, 145, 158]. This region begins at the end of the ion saturation region and ends at the plasma potential $\left(V_{S}\right)$, also referred to as the space potential. The plasma potential is the point at which the bias voltage is large enough that no ions reach the probe, and the current is strictly attributed to electrons [30, 31, 141, 158]. The plasma potential ideally presents itself as a "knee" in the current-voltage plot, but this is often obscured due to error, making it difficult to determine $[30,31,158]$. The floating potential $\left(V_{f}\right)$, the bias voltage at which an equal number of ions and electrons reach the probe, also lies in this region between the ion saturation current and the plasma potential. The transition region of the current-voltage plot can be used to determine the electron temperature as well as the electron energy distribution function.

The third region of the probe characteristic is the electron saturation region (depicted as the right portion of Fig. 2.4) [30, 31, 85, 91, 133, 141, 145, 158]. In this region, the probe is biased positive of the plasma potential and only electrons reach the probe $[30,31,85,91,133,141,145,158]$. The electron saturation region can create significant problems when used for measurements because of plasma perturbation $[30,31,85,91,127,133,141,145,158]$. As the electrons are drawn from the plasma to the probe, a current is drawn from the bias voltage supply to the plasma causing perturbation in the plasma around the probe [127]. Plasma perturbation can cause significant changes to the electron temperature and density of the plasma as well as other plasma characteristics [126, 152]. The perturbation can also cause damage to the probe [30, 31, 141, 158]. The electron saturation region can be used to determine the particle density of the plasma through the determination of the electron saturation current $\left(I_{e}\right)$.

The current-voltage characteristic can be used to determine characteristic plasma parameters. The electron temperature is determined by finding the inverse of the slope of the semi-logarithmic plot of current in the transition region $[30,141]$. The particle density $\left(n_{e},\left[\mathrm{~m}^{-3}\right]\right)$ assumed to be equal for ions 
and electrons in the bulk of the plasma, can be found using multiple methods. If the electron saturation current is known the density can be determined through (2.5), a function of the electron saturation current $\left(I_{e},[\mathrm{~A}]\right)$, probe area $\left(A,\left[\mathrm{~m}^{2}\right]\right)$, electron mass $(m,[\mathrm{~kg}])$, and electron temperature $\left(k T_{e},[\mathrm{eV}]\right)[31,99,158]$. The electron density can also be determined by integrating over the electron energy distribution function [99]. A third, method of determining the electron saturation current is through (2.6), a function of the ion saturation current $\left(I_{i},[\mathrm{~A}]\right)$, sheath area $\left(S,\left[\mathrm{~m}^{2}\right]\right)$, Coulomb's constant $(e,[\mathrm{C}])$, ion mass $(M,[\mathrm{~kg}])$, and electron temperature $\left(k T_{e},[\mathrm{eV}]\right)$. Each of these methods results in error in the electron density measurement, with a minimum of $10-20 \%$ [30, 31]. The electron energy distribution function is proportional to the second derivative of the transition region of the current-voltage characteristic [30, 31]. If the plasma is Maxwellian, the electron energy distribution function will be of the form (2.7), where $v$ is the ion velocity and $v_{t h}=\sqrt{2 k T_{e} / m}$. Typically the distribution function is assumed to be Maxwellian but can be non-Maxwellian as well which will result in a different normalizing coefficient [30, 31]. The floating voltage is found at the point where the measured current is equal to zero on the current-voltage plot, and shows where equal numbers of ions and electrons are collected [30, 31, 122, 141, 142]. The plasma potential can be found at the "knee," but this is not always obvious [30, 31]. It can also be estimated using (2.8) a function of the floating voltage $\left(V_{f},[\mathrm{~V}]\right)$, electron temperature $\left(k T_{e},[\mathrm{eV}]\right)$, elementary charge $(e,[\mathrm{C}])$, ion mass $(M,[\mathrm{~kg}])$, and electron mass $(m,[\mathrm{~kg}])[31,158]$. Depending on the plasma, this estimation is not always accurate [30, 31, 158]. Typically, the plasma potential cannot be determined using the electrostatic probe method [158].

$$
n_{e}=\frac{I_{e}}{A} \sqrt{\frac{2 \pi m}{k T e}}
$$




$$
\begin{gathered}
n_{e}=\frac{I_{i}}{0.61 S e} \sqrt{\frac{M}{k T_{e}}} \\
f(v)=\left(\frac{1}{v_{t h} \sqrt{\pi}}\right) \exp \left(\frac{-v}{v_{t h}}\right) \\
V_{S}=V_{f}+\frac{k T_{e}}{2 e} \ln \left(\frac{2 M}{\pi m}\right)
\end{gathered}
$$

\subsubsection{Double Electrostatic Probes}

In cases where a single probe would be too large, and cause excessive heat loads resulting in a change in the plasma parameters or an oscillating plasma, a double probe can be used [85, 92, 95, 109, 110, 118, 125, 133, 141, 155]. Fig. 2.5 shows a sample symmetrical double probe system [85, 92, 95, 109, 110, 118, 125, $133,141,155]$. In a symmetrical double probe system, each probe is identical; in an asymmetrical probe system one probe is on the order of 100 times the size of the other $[85,92,95,109,110,118,125,133,141,155]$. Both systems can be used to measure the electron temperature, but the symmetrical system is better for measuring the electron density while the asymmetrical system is better for measuring the electron energy distribution function. In both systems, the plasma completes the circuit between the two probes, while the probes remain isolated from ground [133].

In a double probe system, the two probes float, resulting in the electron current flowing through the positive probe and the ion current flowing through the negative probe allowing for the measurement of the ion saturation between the two $[85,110,133]$. This set-up is preferable to single probes in that it results in limited plasma perturbation as the probes are always negative of the plasma potential [85, 110, 133].

A bias voltage is swept on both probes and the current response is measured, as shown in Fig. 2.5; this results in a current-voltage trace which is the same as 
the single probe method consisting of ion saturation, transition, and electron saturation regions. In double probe systems, the electron temperature and electron energy distribution functions are found using the same method as the single probe, and the electron density is found using (2.6) because the ion current is measured [109, 110, 133, 155]

An advantage to the double probe system is that the current draw to the probe is limited by the size of the probes [110, 133]. Also the perturbation by the probe is limited because the maximum current is the sum of the ion and electron saturation currents (which is generally small) [133]. The main drawbacks to the double probe system is the difficulty associated with ensuring that the probes are kept floating and the system only measures the electrons from the high energy tail of the plasma, giving an inaccuracy in the electron temperature measurement [110].

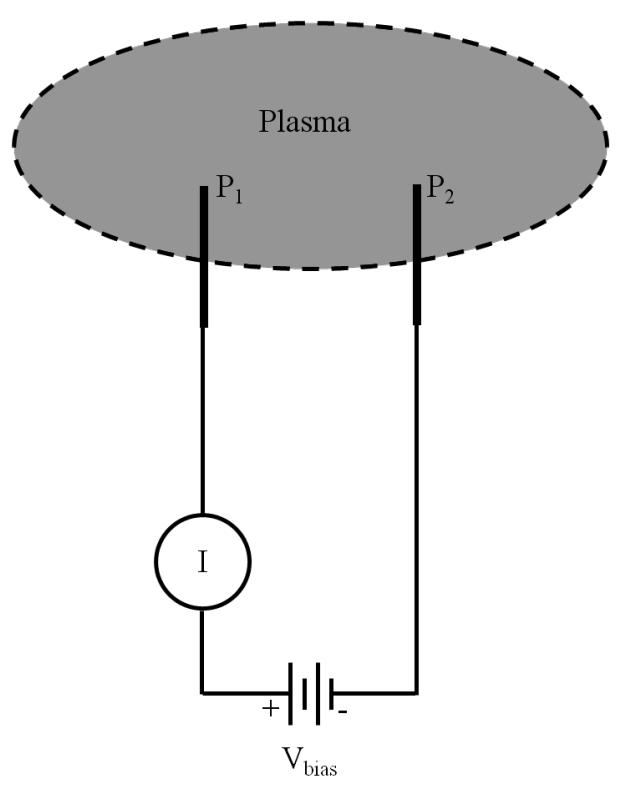

Figure 2.5: Schematic of a symmetrical double electrostatic probe system. 


\subsubsection{Triple Electrostatic Probes}

A triple probe system uses three identical probes at different static voltages, and is recommended in plasmas where a swept bias voltage is not possible or where the plasma contains a frequency component, such as RF plasmas [84, 89, 92, 102, 106, 107, 109-111, 119, 137]. By not requiring a voltage sweep, as in the previous single and double probe methods, the triple probe can be used to instantaneously measure the temperature and density of the plasma. This type of measurement is ideal in situations where the plasma is oscillating, such as an RF plasma [84, 89, 92, 102, 106, 107, 109-111, 119, 137], or plasmas that would cause high thermal loads on a single or double probe system [95]. Triple probe systems also allow for direct and instantaneous display of the plasma parameters, which is convenient when several different input parameters are being tested and reduce the time required for testing [84, 89, 92, 102, 106, 107, 109-111, 119, 137].

A sample triple probe system is shown in Fig. 2.6 with the static potentials of each probe with reference to the plasma potential $\left(V_{s}\right)$ shown in Fig. 2.7 [84, 89, 92, 102, 106, 107, 109-111, 119, 137]. Probe 2 is left floating, resulting in no current flowing through the probe, and allowing for a direct measurement of the floating potential. Probes 1 and 3 are connected through a static bias voltage, similar to a floating double probe. As is the case with the floating double probe, no net current flows through the probes, resulting in low perturbation.

In the direct display triple probe system, measurements are made using three assumptions [84, 92, 102, 106, 107, 110, 147]. First, the plasma electron distribution is assumed to be Maxwellian. Second, the probes are assumed to be separated by a distance greater than the sheath to prevent interaction between probes. Third, the mean free path is assumed to be greater than the ion sheath and the probe radius, implying a collisionless probe sheath. Based on these assumptions the plasma is assumed to be operating in the orbital motion limited (OML) regime. Given the assumptions, the electron temperature $\left(T_{e},[\mathrm{eV}]\right)$ is 
found using (2.9) a function of the measured voltage between the floating probe $\left(\mathrm{P}_{2}\right)$ and the positive floating probe $\left(\mathrm{P}_{1}\right)\left(V_{d 2},[\mathrm{~V}]\right)$, and the fixed bias voltage $\left(V_{d 3},[\mathrm{~V}]\right)[84,92,102,106,107,110,147]$. The fixed applied bias voltage $\left(V_{d 3}\right)$ should be chosen such that it ensures the system is in ion saturation, between five and ten $V_{d 2}$, with the voltages under the lower bound corresponding to underestimation and over the upper bound resulting in overestimation of the electron temperature [92].

$$
\frac{1-\exp \left(\frac{-e V_{d 2}}{T_{e}}\right)}{1-\exp \left(\frac{-e V_{d 3}}{T_{e}}\right)}=\frac{1}{2}
$$

Once the electron temperature has been determined, the electron density can be estimated using the measured ion saturation current. The ion saturation current $(I,[\mathrm{~A}])$ is the current flowing from $\mathrm{P}_{1}$ to $\mathrm{P}_{3}$. The electron density $\left(n_{e}\right.$, $\left.\left[\mathrm{m}^{-3}\right]\right)$ is estimated using $(2.10)$ a function of the measured ion current, the ion mass $(M,[\mathrm{~kg}])$, probe surface area $\left(A,\left[\mathrm{~m}^{2}\right]\right)$, measured voltage between $\mathrm{P}_{2}$ and $\mathrm{P}_{1}\left(V_{d 2},[\mathrm{~V}]\right)$, and the estimated electron temperature $\left(T_{e},[\mathrm{eV}]\right)[84]$. If the mean free path is not greater than the sheath or the probe radius, the electron temperature is still calculated using the same equation, but the electron density must be corrected to include collisions in the sheath [111, 147].

$$
n_{e}=1.05 \times 10^{15} \frac{I \cdot(M)^{\frac{1}{2}}}{\left(T_{e}\right)^{\frac{1}{2}} A\left[\exp \left(\frac{V_{d 2}}{T_{e}}\right)-1\right]}
$$

The triple probe method is useful in situations where a bias voltage sweep is not possible $[84,85,111,147]$. Because the system floats, a relatively small plasma perturbation is expected [85, 89, 92, 102, 106, 107, 109-111, 119, 137]. Also, oscillation of the plasma (such as those in RF and microwave plasmas) is not a source of error in triple probes as it is in single probes because the system floats with the oscillations of the plasma [85, 89, 92, 102, 106, 107, 109-111, 119, 137]. However, triple probe systems are unable to provide any information on the 
electron energy distribution function or the plasma potential because the probe bias voltage is not swept $[85,89,92,102,106,107,109-111,119,137]$.

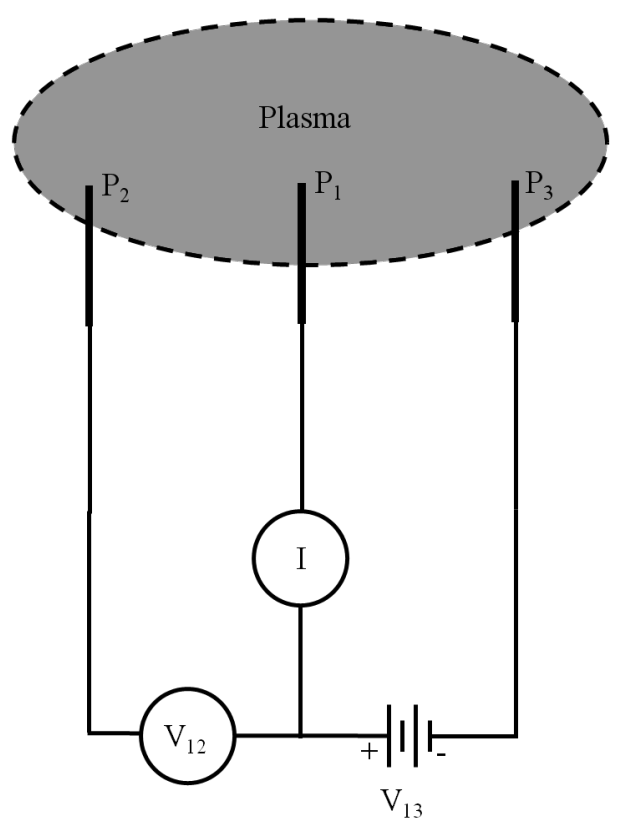

Figure 2.6: Schematic of a symmetrical direct display triple probe system.

\subsubsection{Ion Trajectories in the Saturation Region}

In the ion saturation current region, the probe potential is sufficiently negative to the plasma potential to cause the ion current to saturate the probe. In this region, the electrons are repelled by the negative voltage while ions are collected. The ions interact with the probe in four different ways as is shown in Fig. 2.8 [130, 134]. In the first interaction (path 1 in Fig. 2.8), the ions pass by the probe and do not contribute to the probe current, while still possibly having a large contribution to the ion density in the probe sheath [130,134]. The second ion interaction (path 2 in Fig. 2.8) is orbital motion limited (OML) in which an ion from a distance is attracted to the probe $[77,80,83,86,93,102,120,124,130$, 134, 150, 151]. The third interaction (path 3 in Fig. 2.8) is an ion that orbits 


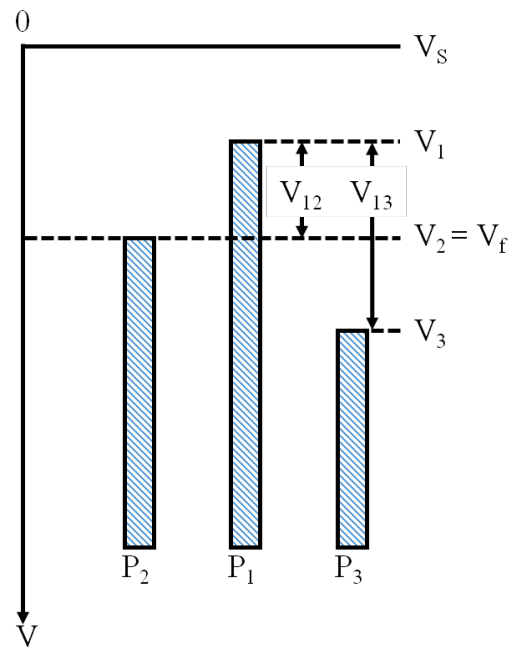

Figure 2.7: Potential of each probe in the triple probe system with respect to the space potential of the plasma.

the probe and can be generated by a collision near the probe $[130,134]$. The fourth ion interaction (path 4 in Fig. 2.8) is a trapped orbit that intercepts the probe surface. An ion will become trapped if the ion energy is less than the probe energy and the orbit of the ion does not hit the probe [128-130, 134, 159]. An ion typically becomes trapped after a collision causing ion energy loss [31, 86, 128-130]. Goree determined that a few collisions can contribute a significant amount of trapped ions which can build indefinitely [159]. Trapped ions can account for increased currents in medium pressure plasmas, because almost all ions which have been in a collision will be collected due to their reduced speed resulting in an overestimation in density if OML theory is used [128-130, 134]. Due to their short lifetime, these trapped ions have a negligible population in the plasma, but can contribute significantly to the current if the collision rate is sufficient $[130,134]$. The ions that pass by the probe and the trapped ions have the greatest contribution to the ion current, and cause a significant error in the density calculation [130, 134].

The error in the electron density measurement when estimated using the ion 
saturation current can be as large as 2 to 100 times the electron density measured using the electron saturation current, with average values reported in the range of an order of magnitude difference [82, 99, 125, 132]. These values have been measured using different methods in comparison with electrostatic probe data including microwave interferometry. Even with the error, the electron density measured using the ion saturation current is still considered a preferable method to the electron saturation current, because of the significant current draw in the electron saturation region [30, 125-127, 130, 135, 148, 152]. The large current draw in electron saturation can result in significant perturbation and melting of the probe [31, 127, 141]. In the electron saturation region, current is introduced into the system from the probe bias voltage source, causing potentially large perturbations in the sheath region resulting in the underestimation of the area affected by the probe [127]. These perturbations can cause unwanted effects in the bulk region of the plasma, including altering the electron temperature and density of the plasma, thereby defeating the purpose of the measurement. However, a measurement in the ion saturation region draws no current from the probe bias, therefore there should be no significant perturbation of the plasma. Because of the predictable nature of the ion trajectories, multiple theories for the correction of the probe data to determine more accurate results of the electron density are available [135, 136, 148, 150, 160-162].

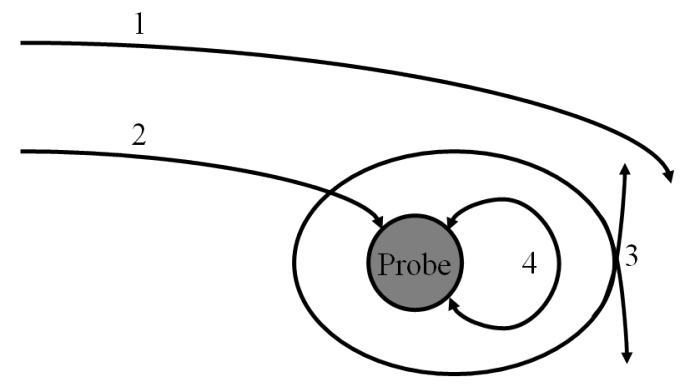

Figure 2.8: The ion trajectories when the probe is biased in the ion saturation region. 


\subsubsection{Theories for Probe Current Correction}

In 1926, Mott-Smith and Langmuir believed that particles reached the probe using the orbital motion limited (OML) theory [150]. Langmuir and Mott-Smith based OML theory on the conservation of angular momentum and energy and is applicable to experiments with Debye numbers less than or equal to three [93, 121]. In OML, they assumed that some particles from the plasma at every energy (and hence speed) will impact the probe and be measured. While this method can be successful in a collisionless plasma in a thick sheath, OML does not account for the absorption radius surrounding the probe, resulting in significant quantities of trapped ions [77]. The trapped ions cause an overestimation of electron density especially when the Debye number is finite. Also, several sources have shown that OML tends to be inaccurate in the ion saturation region, where just a few collisions with neutrals can result in the total destruction of OML [116].

In 1949, Bohm generated a model to improve upon the theory of MottSmith and Langmuir [78, 163]. The Bohm model included monoenergetic ions and Maxwellian electrons in the thin sheath of a plane probe [163]. By using a thin sheath, ionization near the probe was negligible and could be ignored. Bohm determined that the ion acceleration toward the probe was caused by the electric field generated by the probe extending into the quasi-neutral region of

the plasma [86, 163]. From his model, Bohm determined that the velocity $\left(\nu_{B}\right)$ of the ion entering the sheath region (commonly referred to as the Bohm velocity) was described by (2.11) where the velocity is a function of Boltzmann's constant $(k)$, the electron temperature $\left(T_{e}\right)$, and the ion mass $(M)$.

$$
\nu_{B} \geq\left(\frac{k T_{e}}{M}\right)^{1 / 2}
$$

In 1957, Allen, Boyd, and Reynolds created a spherical probe model which simplified previous theories by claiming that cold ions in an unperturbed plasma do not follow OML, but are drawn to the probe radially [160]. The theory 
generated a universal set of density solutions by solving Poisson's equation for ions between the probe and infinity (the bulk of the plasma) [31]. This probe theory was extended in 1965 by Chen to include cylindrical probes [157]. This probe theory is applicable to plasma-probe relationships with Debye numbers ranging from 0.5 to 70 and Knudsen numbers ranging from zero to infinity [78, $135,136,157]$.

In 1959, Bernstein and Rabinowitz extended the Langmuir and Mott-Smith theory [161]. The Bernstein and Rabinowitz theory combined OML with the thick sheath and is applicable to plasmas in the five to 15 Debye number range. They included the sheath width in their model because when the Debye number is small the ion saturation current increases as the probe voltage becomes more negative. This increase in ion current is assumed to be caused by the increase in the size of the sheath around the probe. They also assumed that ions orbiting the probe contributed twice to the density calculation, while ions that strike the probe only contributed once [30]. Chen also extended this theory for cylindrical probes [157].

In 1969, Laframboise extended the Bernstein and Rabinowitz and Chen theories to include Maxwellian distribution of electrons [104]. Laframboise's calculation applies to a wide range of Debye numbers, ion to electron temperature ratios, and probe potentials $[80,104,114,135,136]$. This calculation demonstrates that the ion current calculated using Maxwellian and monoenergetic ion energy distributions show very little difference from each other when the Debye number is less than three, and the sheath can still be considered to be OML [104, 114, 135, 136]. Through microwave interferometry, the Bernstein, Rabinowitz, and Laframboise method has been proven accurate in cases where the ion mean free path is greater than the probe radius [114].

Chou, Talbot, and Willis suggested that collisions with neutrals in the sheath will result in a decrease in the ion and electron current to the probe. To correct for 
this, they calculated a collision factor and applied it to Laframboise's collisionless probe current. This was calculated in more detail by Talbot and Chou and Kirchoff, Peterson, and Talbot [103].

Chen compared the theories of Allen, Boyd, and Reynolds (ABR) and Bernstein, Rabinowitz, and Laframboise (BRL) with the electron density measured using microwave interferometry [30, 81, 157]. Through this experiment he determined that because ions orbiting the probe are neglected the estimate is lower than the actual value. Chen also determined that the density estimated through the BRL theory will exceed the actual value because of the assumption of more orbiting than should exist. The geometric mean of the values estimated with $\mathrm{ABR}$ and BRL agrees well with the density determined through microwave interferometry.

In 1977, Zakrzewski and Kopiczynski showed that when the Debye number is less than three and the Knudsen number is in the range from 0.5 to infinity the ion current was impacted by two factors: the reduction of current due to elastic scattering and the increase of current due to the destruction of OML conditions [148]. This was confirmed experimentally through comparisons with microwave interferometry [121]. This was extended by Tichy, et al. and was tested over a wide range of operating conditions [135, 136, 146, 154]. Numerical simulations comparing different probe theories showed that the theory presented by Tichy provided the most accurate results especially in the transition pressure region [98].

\subsubsection{Error in Electrostatic Probe Measurements}

While electrostatic probes have been shown to be a sound method for estimating plasma characteristics, this type of measurement can have non-ideal effects on the plasma [75, 85, 90, 91, 115, 127, 133, 136, 142, 164]. Errors involving the probe include those caused by the probe size, geometry, temperature, and 
surface. Other sources of error include using the wrong probe theory and unaccounted for plasma oscillations.

The probe size can have multiple effects on the plasma. If the probe is too large, more particles are drawn to the probe than are lost due to recombination, convection, and diffusion [133]. A probe that is too large will also result in rounding off current-voltage trace near the plasma potential effecting the measurement of the plasma potential, electron energy distribution function, and the plasma density [75, 133, 136, 139, 140].

Inherently, the probe will cause some disturbance of the plasma, but probe geometry can also cause excess perturbation [78, 90, 91, 141]. The probe geometry has the greatest potential for error when using a spherical probe because the sphere is attached to a wire which acts like a cylindrical probe $[105,133,141]$. The best method for removing geometry errors is to attach the probe directly to the wall, or minimize the size of the probe holder to smaller than the mean free path $[94,141]$. Error resulting from the probe geometry has the greatest impact on the electron density measurement [79, 91].

The temperature of the probe can also cause perturbations and recombination in the plasma [127]. The probe is at a lower temperature than the plasma, which lessens the mobility of the particles near the probe leading to a lower temperature measurement while recombination would result in an overestimation of the plasma electron density [105, 127, 133].

Error in probe measurements can also occur as the result of changes in the surface of the probe. Probe surface changes can include changes in the work function and resistance due to contamination or melting of the probe [75, 78, 133, 136, 141]. These changes have the greatest impact at the plasma potential and have a lesser affect near the floating potential [141]. This layer of contamination acts as additional resistance and can result in underestimation of the collection area of the probe [133]. If the work function is not even across the probe surface, 
a small uncertainty can be expected in the plasma potential, typically resulting in broadening in the current-voltage characteristic of approximately $0.1 \mathrm{eV}$ in the electron temperature and uncertainty in the electron energy distribution function [91]. Work function effects generally appear as hysteresis in the currentvoltage trace [141]. A dirty probe can result in plasma contamination and secondary electron emission [91]. When secondary electrons are generated in ion saturation, the secondary electrons are accelerated away from the probe causing ionization as they move towards the bulk of the plasma [141]. Secondary electrons can cause decreased temperature estimations as well as changes in the density estimations [75, 91, 136].

Error in electron density measurements can also be associated with using the wrong theory $[86,132,135,136]$. It is important to check the Knudsen and Debye number ranges for the theory chosen, and the collisionality of the plasma [132]. Assuming OML when the plasma contains even a few collisions can result in an error of up to an order of magnitude in the electron density.

Plasma oscillations, such as those found in RF and microwave plasmas, can cause error in the current-voltage characteristic which propagates error in the estimated plasma parameters [30, 31, 78, 100, 138, 141, 153]. When an oscillation in the plasma exists, but is unaccounted for, the current-voltage characteristic is broadened in the transition region [30,31, 131, 141]. This broadening can lead to an overestimation of the electron temperature and an underestimation of the floating potential [30, 31, 131, 141]. Several methods of accounting for oscillations exist including floating multi-probe systems (such as double and triple probe systems), filtering circuits, and time averaging (such as box car averaging) [30, $31,100,131,138,141,153,156]$. 


\subsection{Hydrogen Ion Concentration}

The chemical kinetics of hydrogen plasmas have been extensively studied through modeling and experiment and have been shown to be a function of the electron density, electron temperature, gas pressure, and plasma generating chamber size [165-168]. At moderate temperatures, hydrogen plasmas are comprised of two neutral species $\left(\mathrm{H}_{2}\right.$ and $\left.\mathrm{H}\right)$ and three positive ionic species $\left(\mathrm{H}^{+}, \mathrm{H}_{2}{ }^{+}\right.$, and $\left.\mathrm{H}_{3}{ }^{+}\right)\left[165\right.$ ? -175]. When suprathermal electrons $\left(\mathrm{T}_{\mathrm{e}} \sim 40 \mathrm{eV}\right)$ are present, negative ions $\left(\mathrm{H}^{-}\right)$are also part of the plasma [169]. Two sets of reactions are considered for a moderate temperature hydrogen plasma reaction, gas phase reactions (shown in Table 2.2) and surface reactions (shown in Table 2.3) [168, 169, 171, 172].

Based on the models and experiments, at pressures greater than 0.02 Torr the dominant ion in the plasma is $\mathrm{H}_{3}{ }^{+}[172,173]$, with concentrations typically being an order of magnitude greater than that of $\mathrm{H}^{+}$and $\mathrm{H}_{2}{ }^{+}$[165? -175]. While $\mathrm{H}_{2}{ }^{+}$ions require the lowest energy for creation, they are quickly eliminated through collisions with $\mathrm{H}_{2}$ which efficiently generates $\mathrm{H}_{3}{ }^{+}$through reaction 10 in Table $2.2[165,166,168,171,176]$. If $\mathrm{H}_{2}{ }^{+}$is the desired dominant ion, the plasma should be operated at temperatures greater than $5 \mathrm{eV}$, and if $\mathrm{H}^{+}$is the desired ion the plasma should be highly collisional $\left(N_{e}<N_{H_{2}} / 10\right.$ where $N_{e}$ is the electron density and $N_{H_{2}}$ is the concentration of hydrogen supplied to the system) [169]. These results remain constant over a range of modeled chamber sizes, gas pressures, electron temperatures, and electron densities [165? -175]. Investigation into the impact of frequency on the plasma show that at frequencies above $1 \mathrm{MHz}$, a decrease in the sheath size and increase in density occurred, but the ion concentration was unaffected [165, 166, 177]. 
Table 2.2: Gas Phase Hydrogen Reactions Considered

\begin{tabular}{ccc}
\hline Reaction Number & Collision Type & Reaction \\
\hline \hline 1 & Electron-Neutral & $\mathrm{H}+\mathrm{e} \rightarrow \mathrm{H}^{+}+2 \mathrm{e}$ \\
2 & Electron-Neutral & $\mathrm{H}_{2}+\mathrm{e} \rightarrow \mathrm{H}^{+}+\mathrm{H}+2 \mathrm{e}$ \\
3 & Electron-Ion & $\mathrm{H}_{2}^{+}+\mathrm{e} \rightarrow \mathrm{H}^{+}+\mathrm{H}+\mathrm{e}$ \\
4 & Electron-Ion & $\mathrm{H}_{2}^{+}+\mathrm{e} \rightarrow \mathrm{H}^{+}+\mathrm{H}^{+}+\mathrm{e}$ \\
5 & Ion-Neutral & $\mathrm{H}_{2}+\mathrm{H} \rightarrow \mathrm{H}_{2}+\mathrm{H}^{+}$ \\
6 & Ion-Neutral & $\mathrm{H}_{2}+\mathrm{H}^{+} \rightarrow \mathrm{H}_{2}++\mathrm{H}$ \\
7 & Electron-Neutral & $\mathrm{H}_{2}+\mathrm{e} \rightarrow \mathrm{H}_{2}^{+}+2 \mathrm{e}$ \\
8 & Electron-Ion & $\mathrm{H}_{3}^{+}+\mathrm{e} \rightarrow \mathrm{H}_{2}^{+}+\mathrm{H}+\mathrm{e}$ \\
9 & Electron-Ion & $\mathrm{H}_{2}^{+}+\mathrm{e} \rightarrow 2 \mathrm{H}$ \\
10 & Ion-Neutral & $\mathrm{H}_{2}^{+}+\mathrm{H}_{2} \rightarrow \mathrm{H}_{3}++\mathrm{H}$ \\
11 & Electron-Ion & $\mathrm{H}_{3}^{+}+\mathrm{e} \rightarrow 3 \mathrm{H}$ \\
12 & Electron-Ion & $\mathrm{H}_{3}++\mathrm{e} \rightarrow \mathrm{H}_{2}+\mathrm{H}$ \\
13 & Electron-Neutral & $\mathrm{H}_{2}+\mathrm{e} \rightarrow 2 \mathrm{H}+\mathrm{e}$ \\
\hline
\end{tabular}

Table 2.3: Surface Hydrogen Reactions Considered

\begin{tabular}{cc}
\hline Reaction Number & Reaction \\
\hline \hline 1 & $\mathrm{H}+$ wall $\rightarrow \mathrm{H}_{2}$ \\
2 & $\mathrm{H}^{+}+$wall $\rightarrow \mathrm{H}$ \\
3 & $\mathrm{H}_{3}^{+}+$wall $\rightarrow \mathrm{H}_{2}+\mathrm{H}$ \\
\hline
\end{tabular}




\section{Chapter 3}

\section{Experimental Set-Up}

This chapter details the experiments used to characterize the piezoelectric transformer plasma source (PTPS) as an ion source for neutron production. An overview of the PTPS operation, including a description of the PTPS, electrical drive circuit, and current diagnostic is provided in Section 3.1. Also presented are the operating conditions for the characterization experiments, as well as the parameters that were varied (including the driving voltage, extraction voltage, hollow anode diameter, and internal pressure of the PTPS). Other experiments described include the high voltage dc system (used to determine if neutrons above background could be measured using the PTPS as the ion source) and a triple electrostatic probe experiment used to determine the electron temperature and particle density of the plasma. It should be noted that some portions of the Experimental Set-Up and Experimental Results chapters were previously included in the final report to Los Alamos National Laboratory [178].

\section{$3.1 \quad$ PTPS}

The PTPS (shown in Fig. 3.1) consists of a $\mathrm{LiNbO}_{3}$ (lithium niobate) cylindrical disk measuring $10 \mathrm{~mm}$ in diameter by $2.0 \mathrm{~mm}$ thick, which was driven through the piezoelectric transformer thickness by an RF driving voltage. 
Lithium niobate is a piezoelectric material with a high melting point, Curie point, and quality factor all of which are required to minimize damage on the disk due to plasma formation [55, 179, 180]. A high voltage is developed on the disk surface through the converse and direct piezoelectric effects. The converse piezoelectric effect is used to establish harmonic modes in the $\mathrm{LiNbO}_{3}$ disk when operated at one of the mechanical resonant frequencies for the transformer. When operated at one of its resonant frequencies, an axial strain is induced through the thickness of the disk by the applied axial electric field, Fig. 3.2a, due to the converse piezoelectric effect. Orthogonal mechanical coupling in the disk resulted in an internal radial stress, Fig. 3.2b, simultaneously resulting in a high voltage on the disk surface from the direct piezoelectric effect $[65,66]$.

The driving electrode was placed on springs to improve clamping uniformity. Improved clamping uniformity has been shown to result in more repeatable clamping conditions on the crystal as measured with the electrical quality factor. A gas was differentially pumped into the high electric field volume between the disk surface and hollow anode, and was ionized, resulting in the formation of plasma inside the PTPS chamber. The ions from the plasma, assumed to be quasineutral, could then be extracted through the hollow anode for the purposes of the experiment.

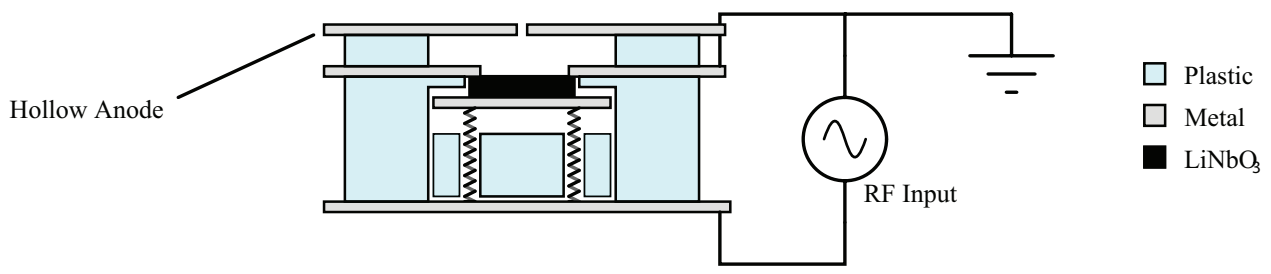

Figure 3.1: Cross-section of the PTPS. 


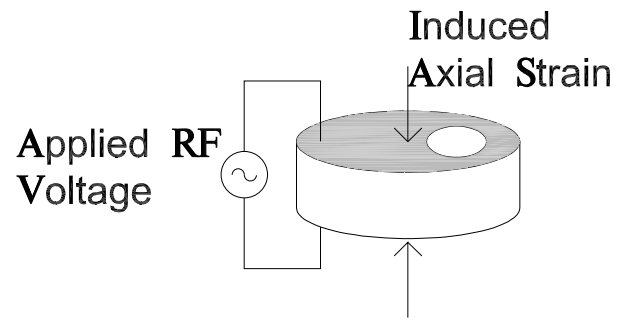

(a)

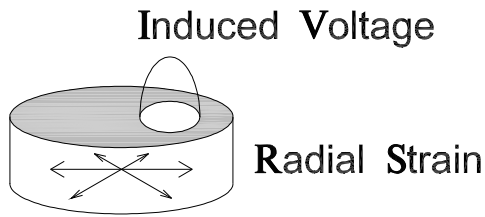

(b)

Figure 3.2: Input response due to the converse piezoelectric effect (a) and output response due to the direct piezoelectric effect (b) of the radial crystal.

\subsection{Ion Source Characterization Experiment}

To determine if the PTPS was a viable ion source for neutron production, the PTPS was characterized using the system shown in Fig. 3.3. The PTPS was characterized as an ion source in a cryogenically pumped vacuum chamber. The rated initial pumping speed of the cryopump for hydrogen was $5000 \mathrm{~L} / \mathrm{sec}$; the background pressure during testing was $5 \times 10^{-5}$ Torr [181]. The internal pressure $P$ of the PTPS was estimated using (3.1). The internal PTPS pressure was a function of the gas flow rate $Q$, the hollow anode radius $a$, the molar gas constant $R$, temperature $T$ (assumed to be room temperature), and molecular mass $M$ [182]. The background pressure was ignored in the calculation because it was five orders of magnitude smaller than the internal pressure.

$$
P=\frac{4 Q}{\pi a^{2}} \sqrt{\frac{M}{3 R T}}
$$

The PTPS was tested as an ion source using both hydrogen and deuterium gases. Neutron production using the PTPS as the ion source used the ${ }^{2} \mathrm{H}(d, n)^{3} \mathrm{He}$ nuclear reaction. For this reaction, the PTPS was used as a source of deuterium ions, so initial ion source characterization was performed with deuterium gas. A functional relationship, described in the ion current model in Section 2.1, was established between the deuterium gas and the relatively less expensive hydrogen; 
subsequent tests used hydrogen. Ion currents were measured at 2.5 Torr internal pressure for deuterium gas and a range from 1.5 to 11.0 Torr for hydrogen gas (depending on the flow rate and diameter of the hollow anode) as calculated using (3.1).

The $\mathrm{LiNbO}_{3}$ disk was operated at a resonant frequency of approximately $385 \mathrm{kHz}$ using a function generator operating in burst mode. The 1000 cycle bursts were repeated with a one second period. The amplitude of the signal produced by the function generator was increased using an RF amplifier. A magnetic transformer was used to improve the power transfer efficiency from the RF amplifier to the $\mathrm{LiNbO}_{3}$ disk by lessening the impedance mismatch between the two. The driving current and voltage to the $\mathrm{LiNbO}_{3}$ disk were monitored on an oscilloscope using a current transformer and a voltage divider, respectively.

An ion beam was accelerated from the PTPS to the copper extraction plate using a negatively biased high dc voltage. Secondary electrons produced by the interaction of the ions with the copper extraction plate were kept from impacting the ion current measurement through the use of a secondary electron suppression grid. The suppression grid was constructed of a copper mesh with a $77 \%$ open area fraction, and was held at a $-25 \mathrm{~V}$ bias relative to the extraction electrode. The suppression grid was placed at a fixed distance $(25 \mathrm{~mm})$ from the hollow anode, and $8 \mathrm{~mm}$ from the extraction electrode. When the gap distance $(d$ in Fig. 3.3) was changed the spacing between the suppression grid and the extraction electrode remained constant.

Extraction voltage magnitudes for ion source characterization experiments ranged from 100 to $2000 \mathrm{~V}$. The extraction voltage $V_{e}$ was introduced to the system through a $100 \mathrm{M} \Omega$ current limiting resistor and across a $10 \mathrm{nF}$ integrating capacitor (depicted as $C_{i n t}$ in Fig. 3.3). The electric field profile of the extraction system was flat in the gap between the extraction plate and the hollow anode as is shown in Fig. 3.4 simulated using COMSOL [183]. 
The extracted ion current was collected on the extraction plate and applied to the integrating capacitor. The charge accumulated on the integrating capacitor was measured from ' $\mathrm{X}$ ' in Fig. 3.3 to ground using a voltage probe and oscilloscope. The ion current $I$ was calculated using (3.2), where the current was a function of the integrating capacitor $C_{i n t}$, the change in voltage $\Delta V$, and the change in time $\Delta \mathrm{t}$. Each data point represents the average of 64 consecutive pulses of ion current collected by the target.

$$
I \simeq C_{\text {int }} \frac{\triangle V}{\triangle t}
$$

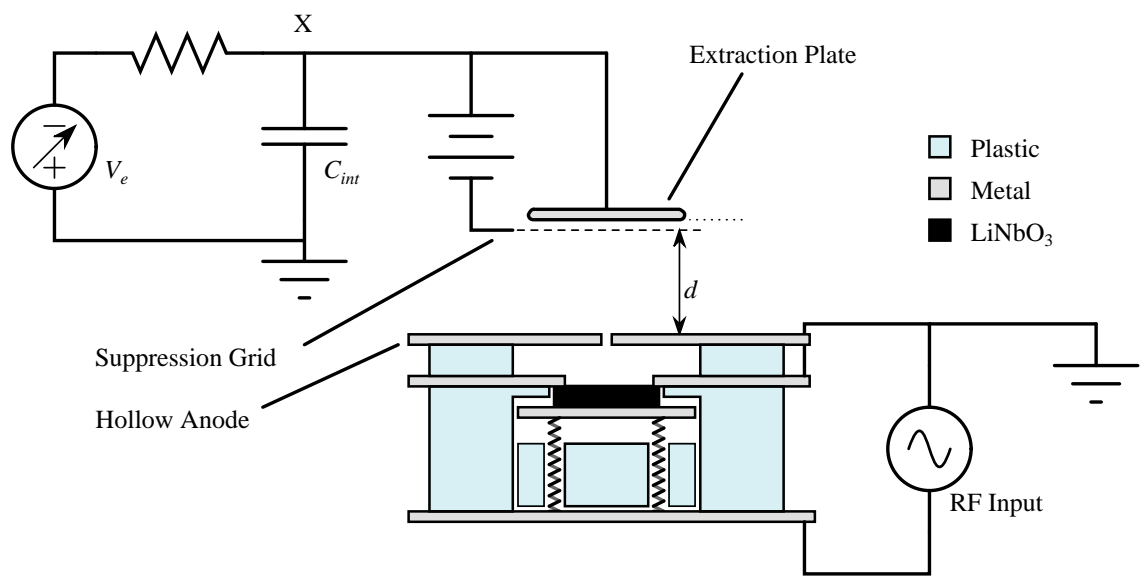

Figure 3.3: Cross section and ion current diagnostic of the PTPS. A LiNbO 3 disk was operated using a RF driving voltage through the crystal thickness. Ions were extracted through the hollow anode and collected on the target electrode. A copper grid was used for secondary electron suppression. Gas was flowed through the dielectric to form a plasma in the area of high voltage generated on the crystal surface. Ion current was collected on the extraction plate, and calculated using the voltage developed on an integrating capacitor.

To be competitive with existing technologies, the PTPS was required to produce ion currents up to $100 \mu \mathrm{A}$. The ion currents from the PTPS were assumed to be controlled by the plasma density and the extraction electric field. The plasma density relates to the neutral gas density and the degree of ionization. Ion 


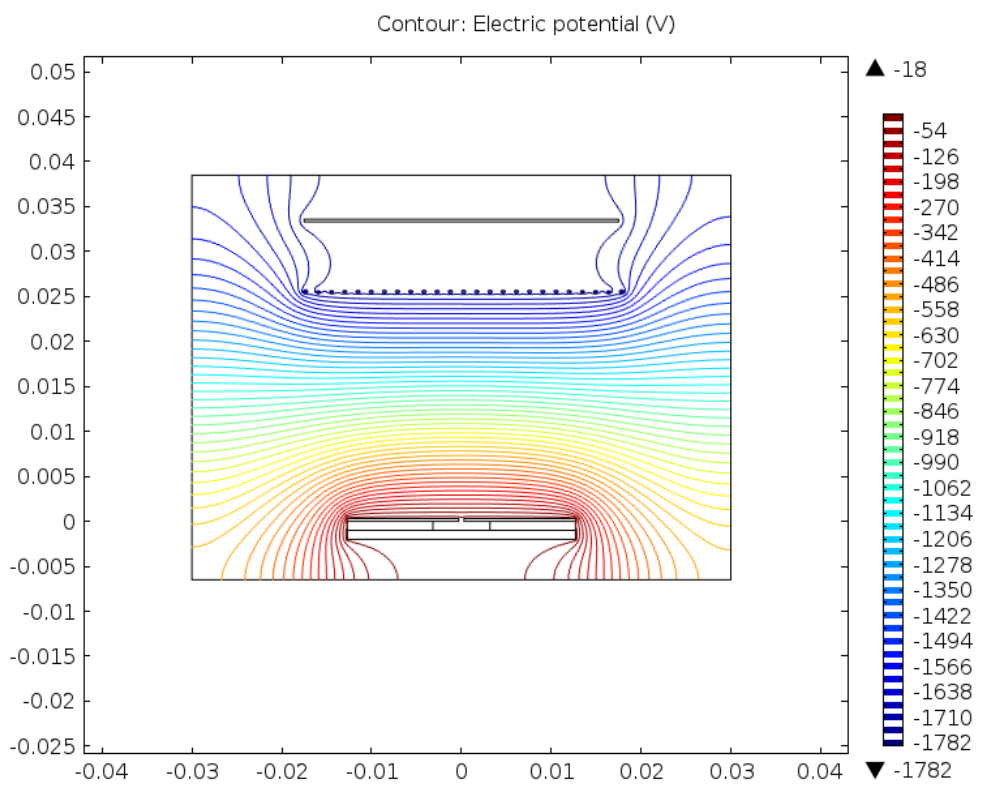

Figure 3.4: Electric field profile of the extraction field of the PTPS ion source characterization experiment.

extraction from the plasma is governed by the dc electric field used to accelerate ions from the plasma to the extraction plate. A parametric sweep was conducted to determine what operating conditions produced the highest ion currents.

The density of the plasma generated using the PTPS is assumed to be a function of the RF driving voltage and the internal pressure of the discharge chamber. The RF driving voltage establishes the discharge voltage on the surface of the $\mathrm{LiNbO}_{3}$ disk through the direct and converse piezoelectric effect. As the RF driving amplitude is increased, the available current density from the piezoelectric transformer is presumed to increase, resulting in greater plasma density.

The internal pressure of the PTPS is influenced by the gas flow rate, the hollow anode radius, and the type of gas used for testing. The gas flow rate into the PTPS determines the amount of neutral gas in the chamber, with a larger gas flow resulting in a larger gas pressure. The hollow anode radius controls the rate of gas removal from the PTPS, producing lower internal pressures at the 
same flow rate with larger radii. The type of gas used for testing, hydrogen or deuterium, influences the average velocity of particles in the gas, via the mass, and, as such, the internal pressure. Increasing the PTPS internal pressure would potentially result in increased plasma density, as well as increased ion currents.

Ion extraction from the PTPS is proportional to the dc voltage applied to the extraction plate and the gap distance between the hollow anode and the suppression grid attached to the extraction plate. The electric field established between the suppression grid attached to the extraction plate and the hollow anode forms a sheath over the hollow anode; this sheath is known as the meniscus. The meniscus serves as a surface area of extraction [184]. The ion current is extracted from the plasma at the meniscus surface. The surface area of the meniscus is controlled by the hollow anode radius and the external electric field. Increased extraction voltages and hollow anode radii would result in increased ion currents.

The depth of the chamber between the hollow anode and the surface of the piezoelectric transformer was varied to determine the effect of plasma generation space on ion current. This experiment used chambers of 3,6, and $9 \mathrm{~mm}$ depth. The ion current should decrease with increasing depth because the electric field in the chamber will decrease with increased gap distance between the piezoelectric transformer and the hollow anode aperture [165].

The PTPS was tested to determine the reduction in ion current over time. The PTPS was operated for 4 to 10 hours in pulsed mode using the same input parameters for 4 to 10 hours to show the change in ion current measured over time. The time experiment was repeated using a second piezoelectric transformer to show the impact of time on multiple piezoelectric transformers.

The amount of ion current reaching the measurement target can be impacted by the collimation of the ion beam toward the target. To increase beam collimation, the meniscus from which ions are extracted should be concave and 
an ion optic could be added to the measurement system [28, 67, 70]. COMSOL simulations were performed to determine the approximate concavity of the meniscus due to the extraction voltage and hollow anode shape. Additional simulations show the affect of adding an optic to the system between the hollow anode and the extraction target [183].

The PTPS was characterized and optimal operating parameters were determined by independently testing each parameter shown in Table 3.1. The results for experiments on type of gas, input RF voltages, dc extraction voltages, hollow anode radii, calculated internal pressures, plasma cavity depth, and operation time are presented in Chapter 4.

Table 3.1: Operating parameters used in ion source characterization experiments

\begin{tabular}{cccc}
\hline Parameter & Variable & Range & Units \\
\hline \hline Input RF Peak Amplitude Voltage & $V_{\text {in }}$ & 200 to 400 & $\mathrm{~V}$ \\
Extraction Voltage & $\left|V_{\text {ext }}\right|$ & 200 to 2000 & $\mathrm{~V}$ \\
Hollow Anode Radius & $a$ & 0.15 or 0.3 & $\mathrm{~mm}$ \\
Gap Distance & $d$ & $10,15,20$, and 25 & $\mathrm{~mm}$ \\
Internal Pressure & $P$ & 1.5 to 11.0 & Torr \\
Ionizing Gas & $g$ & hydrogen or deuterium & \\
Chamber Depth & - & 3,6, and 9 & mm \\
Operating Time & $\mathrm{t}$ & 4 to 10 & hours \\
\hline
\end{tabular}

\subsection{Neutron Experiment}

The PTPS was tested as an ion source for neutron production using the neutron generation system described in Fig. 3.5. The PTPS was integrated into a system using a Hippotronics PP100-5 high voltage supply to provide an accelerating potential to the target. The target was placed in a holder designed for field shaping. As illustrated in Fig. 3.5, the neutron generator used the D-D $\left({ }^{2} H(d, n)^{3} H e\right)$ reaction for neutron production. The PTPS was operated as a deuterium ion source, with the deuterium ion beam impinging on a deuterium- 
doped titanium target. Ions were extracted by the high voltage power supply through a $0.3 \mathrm{~mm}$ radius hollow anode. The input to the dc power supply was regulated using an autotransformer, and the voltage on the target was verified using an x-ray detector placed outside the vacuum. The autotransformer used to control the dc voltage was operated at 50 to $90 \%$ of its full capacity. Fig. 3.6 shows the x-ray measurement of the accelerating voltages used for this experiment. The voltage on the target can be determined for each percent of operation of the auto-transformer by looking at the x-ray energy at the minimum counts. For example, at $50 \%$ of the auto-transofmer operation the voltage on the target was approximately $57 \mathrm{kV}$. The neutron detector was a solid-state RDT neutron detector with a $10^{-7}$ gamma detector efficiency.

The neutron experiment was used to test if the PTPS ion current is sufficient for neutron production above background and to determine the impacts of PTPS operating parameters on the neutron count rate. The PTPS was operated under the series of input parameters shown in Table 3.2, and accelerating voltages ranging from 50 to $100 \mathrm{kV}$.

Table 3.2: Test matrix for PTPS-DC testing.

\begin{tabular}{cccccc}
\hline Test & $\mathrm{A}$ & $\mathrm{B}$ & $\mathrm{C}$ & $\mathrm{D}$ & $\mathrm{E}$ \\
\hline \hline Variable & Control & Drive Voltage & Internal Pressure & Rep Rate & $\mathrm{C} \mathrm{\&} \mathrm{D}$ \\
Internal Pressure & $3 \mathrm{Torr}$ & $3 \mathrm{Torr}$ & 4 Torr & 3 Torr & 4 Torr \\
Drive Voltage & $275 \mathrm{~V}$ & $325 \mathrm{~V}$ & $275 \mathrm{~V}$ & $275 \mathrm{~V}$ & $275 \mathrm{~V}$ \\
Rep Rate & $4 \mathrm{~Hz}$ & $4 \mathrm{~Hz}$ & $4 \mathrm{~Hz}$ & $8 \mathrm{~Hz}$ & $8 \mathrm{~Hz}$ \\
\hline
\end{tabular}

\subsection{Triple Langmuir Probe Experiment}

The electron temperature and particle density of the plasma were approximated using the direct display triple Langmuir probe system shown in Fig. 3.7 with the operating parameters shown in Table 3.3. In this system, three identical cylindrical electrostatic probes with $2.5 \mathrm{~mm}$ length and $0.15 \mathrm{~mm}$ radius were 


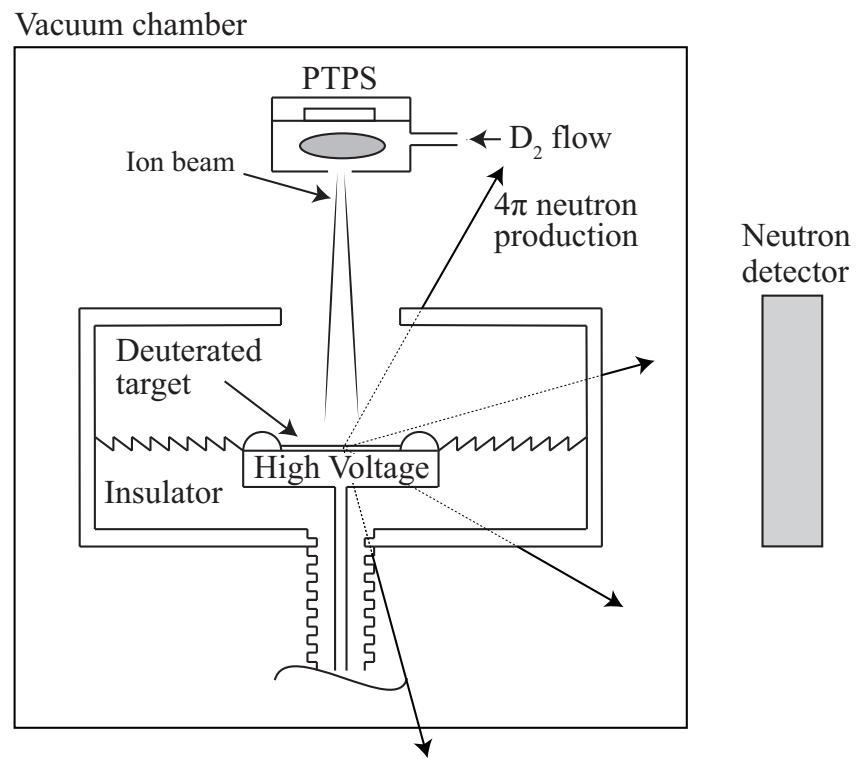

Figure 3.5: Schematic diagram of the PTPS-DC neutron generation system.

inserted into the plasma. The probe length was chosen such that the Debye number was between 0 and 1 , which has been shown to provide the best accuracy for electron temperature measurements [88].Tungsten probes were used because tungsten has been shown to result in low secondary electron emission [78]. One probe, $P_{2}$, had no applied voltage and thus remained at the plasma floating voltage. The other two probes, $P_{1}$ and $P_{3}$, were biased using a fixed dc voltage.

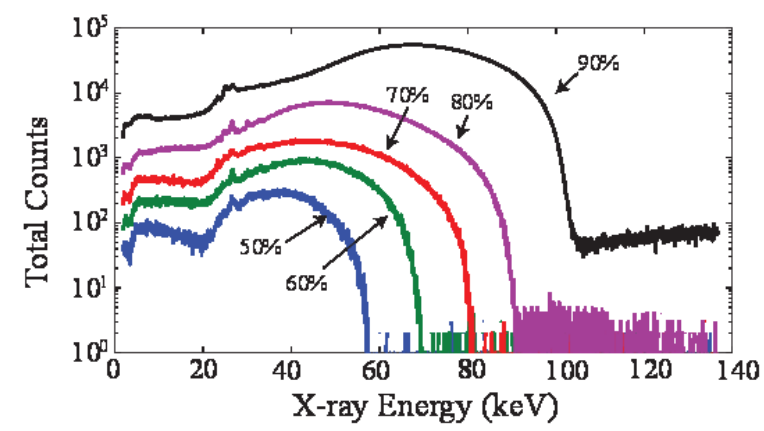

Figure 3.6: X-rays measured at auto-transformer stepping ratios ranging from 50 to $90 \%$ of the total dc voltage supply. 
The voltage difference between the floating probe $\left(P_{2}\right)$ and the positively biased probe $\left(P_{1}\right)\left(V_{d 2}\right.$ in Fig. 3.7) was used to calculate the electron temperature ( $T_{e}[\mathrm{eV}]$ ) using (2.9). In (2.9) $V_{d 3}[\mathrm{~V}]$ is the applied bias between $P_{1}$ and $P_{3}$. The applied bias voltage, $V_{d 3}$, was chosen so that it was between five and ten times $V_{d 2}$, which has been shown to provide accurate electron temperature measurements [92].

Probes $P_{1}$ and $P_{3}$ were connected through the bias voltage $V_{d 3}$, resulting in equal current $(I[\mu \mathrm{A}])$, flowing through each probe $[84,85,110,147]$. The electron density of the plasma, $n_{e}\left[\mathrm{~m}^{-3}\right]$, was calculated using (2.10) where the current is measured using a $10 \mathrm{k} \Omega$ current viewing resistor [108, 110, 156], $M$ is the effective atomic or molecular wight of the ions, $T_{e}[\mathrm{eV}]$ is the plasma electron temperature, and $A\left[\mathrm{~mm}^{2}\right]$ is the area of a single electrostatic probe.

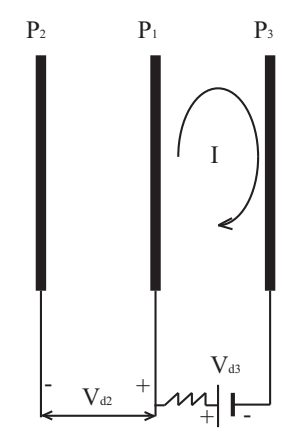

Figure 3.7: Schematic diagram of the triple probe diagnostic for the PTPS.

Table 3.3: Triple probe operating parameters.

\begin{tabular}{ccc}
\hline Parameter & Value & Units \\
\hline Resistor & 10 & $\mathrm{k} \Omega$ \\
$\mathrm{V}_{\mathrm{d} 3}$ & 19.41 & $\mathrm{~V}$ \\
Electrostatic Probe Radius & 0.15 & $\mathrm{~mm}$ \\
Electrostatic Probe Length & 2.5 & $\mathrm{~mm}$ \\
Electrostatic Probe Area & 2.5 & $\mathrm{~mm}^{2}$ \\
\hline
\end{tabular}

These plasma electron temperature and density calculations use the orbital 
motion limited (OML) theory. The OML theory makes three assumptions, as mentioned in Chapter 2. First, the plasma is assumed to have a Maxwellian electron energy distribution function [88, 125, 147, 154]. Second, the ion mean free path is greater than the ion sheath thickness of the probe and the probe radius, indicating no interaction between particles in the sheath surrounding the probe $[77,80,83,84,93,102,120,124,150,151]$. The third assumption is that the thickness of the ion sheath is smaller than the separation between the probes to prevent interaction between probes $[84,89,92,102,106,107,109-$ $111,119,137,147]$. In the PTPS plasma, the first $[88,147,154]$ and third assumptions are assumed to be true. The mean free path, however, is smaller than the sheath thickness because the PTPS was operated in the transitional collisionality region, resulting in error in the particle density calculations using the OML method. Chung, Talbot, and Touryan showed that OML theory can be used in the transition region of plasma collisionality, if collisions in the probe sheath are taken into account [86].

The method established by Tichy, et. al. has been shown to effectively correct for errors caused by collisions in the plasma in the transition collisionality region $[98,116,135,136,154,164]$. This method takes into account error caused by elastic scattering and destruction of OML conditions, and combines multiple electrostatic probe theories to estimate the electron density [98, 116, 135, 136]. First the ion saturation current $\left(I_{i},[\mathrm{~A}]\right)$ was determined through the previously described experiment and normalized using a factor $\left(I_{i}^{*}\right)$ which accounts for elastic scattering and destruction of OML conditions.

The rates of ion current to the probe from destruction of OML conditions and elastic scattering are dependent on the number of collisions in the sheath [135, $136,148,157]$. The rate of ion current caused by the destruction of orbital motion $\left(\gamma_{1}\right)$ is a function of the ratio of the current determined through the Allen, Boyd, and Reynolds (ABR) and Bernstein, Rabinowitz, and Laframboise 
(BRL) theories $[135,136,154]$. If the ion temperature is much less than the electron temperature, the BRL current can be expressed using (3.3), where $\eta$ is a function of the floating potential $\left(V_{f}\right)$ normalized to the voltage equivalent of the electron temperature $\left(V_{T}=k T_{e} / e\right)\left(\eta=V_{f} / V_{T}+10\right)[136,162,185]$. The ABR current can be approximated using (3.4), which is a function of the Debye number $\left(D_{\lambda}=\lambda_{D} / r_{p}\right)$ calculated using the uncorrected electron density, estimated electron temperature, and the normalized floating potential $(\eta)$. The average number of collisions $\left(X_{i}\right)$ in the sheath can be approximated using (3.5) which is a function of the sheath width $(S,(2.3))$ and the ion mean free path $\left(\lambda_{i},(2.4)\right)[135,136,148,154,157]$. The rate of ion current caused by the destruction of orbital motion can be found using either (3.6) for $X_{i}<1$ (nearly collisionless) or (3.7) for $X_{i} \geq 1$, based on the number of collisions in the sheath. The rate of reduction of ion current due to scattering in the sheath $\left(\gamma_{2}\right)$ is a function of the collisions in the sheath, and can be calculated using (3.8), if $X_{i}<1$ and (3.9) if $X_{i} \geq 1$.

$$
\begin{gathered}
I_{B R L}^{*}=\frac{2}{\sqrt{\pi}} \sqrt{\eta} \\
I_{A B R}^{*}=\left(\left(D_{\lambda}+0.6\right)^{0.05}+0.04\right) \cdot\left(\frac{\eta}{0.09 \cdot\left[\exp \left(-D_{\lambda}^{-1}\right)+0.08\right]}\right)^{\left(D_{\lambda}+0.31\right)^{-0.6}} \\
X_{i}=\frac{S}{\lambda_{i}} \\
\gamma_{1}=1+\left(\frac{I_{A B R}^{*}}{I_{B R L}^{*}}-1\right) \cdot X_{i} \\
\gamma_{1}=\frac{I_{A B R}^{*}}{I_{B R L}^{*}}
\end{gathered}
$$




$$
\begin{aligned}
\gamma_{2} & =\frac{\left(3-2 \exp \left(-X_{i}\right)\right)}{\left(1+2 X_{i}\right)} \\
\gamma_{2} & =\frac{\left(3-2 \exp \left(-X_{i}\right)\right)}{\left(2\left(1+X_{i}\right)\right)}
\end{aligned}
$$

The electron density of the plasma can be determined through normalizing the measured ion currents using the normalized dimensionless ion current. The normalized dimensionless ion current $\left(I_{i}^{*}\right)$ can be determined by multiplying the two rates of change for the ion current by the BRL ion current, as shown in (3.10). The electron density of the plasma (taking into account collisions and scattering) can be estimated using (3.11), which is a function of the measured ion saturation current $(I,[\mathrm{~A}])$, normalized dimensionless ion current $\left(I_{i}^{*}\right)$, area of a single probe $\left(A,\left[\mathrm{~m}^{2}\right]\right)$, elementary charge $(e,[\mathrm{C}])$, ion mass $(M,[\mathrm{~kg}])$, and estimated electron temperature $\left(k T_{e},[\mathrm{eV}]\right)$.

$$
\begin{gathered}
I_{i}^{*}=\gamma_{1} \gamma_{2} I_{B R L}^{*} \\
n_{e}=\frac{I}{I_{i}^{*} A e} \sqrt{\frac{2 \pi M}{k T_{e}}}
\end{gathered}
$$

Several different parameters were tested to determine their affect on the plasma electron temperature and density, as shown in Table 3.4. Based on the ion current model in (2.1) the electron temperature and density were assumed to be most affected by the RF input voltage and internal pressure. The dc extraction voltage was also tested to ensure that it has no affect on the characteristic parameters of the plasma. Fig. 3.8 shows the cross section of the system used for the triple probe experiments. The three probes were each placed $0.6 \mathrm{~mm}$ from the surface of the piezoelectric transformer ( $d$ in Fig. 3.8) so that each probe was sampling the same position in the plasma [119]. The PTPS cavity had a depth of $0.9 \mathrm{~mm}$ to prevent breakdown between the electrostatic probes and either the 
grounded hollow anode or the piezoelectric transformer. The extraction plate was located $25 \mathrm{~mm}$ from the $0.3 \mathrm{~mm}$ radius hollow anode. Hydrogen gas was flowed into the PTPS cavity to provide internal pressures ranging from 0.75 to 1.25 Torr resulting in a mean free path ranging from 0.188 to $0.113 \mathrm{~mm}$ and Knudsen numbers ranging from 1.25 to 0.75 . Based on the internal pressure, ion mean free path, and ion Knudsen number for these experiments, the PTPS was operating in the transition region. The PTPS was operated at RF input voltage amplitudes ranging from 175 to $350 \mathrm{~V}$ and ions were extracted at negative voltages ranging from 700 to $2000 \mathrm{~V}$.

The current was measured using $10 \mathrm{M} \Omega$ oscilloscope probes across the $10 \mathrm{k} \Omega$ current sensing resistor. The voltages measured on the electrostatic probes were only present when the PTPS was generating plasma, as is shown in Fig. 3.9. The pulses were averaged 64 times and the mean of the voltage was measured to account for any oscillations in the measurement. Because the PTPS generates a glow discharge plasma, the electron density is expected to be in the range of $10^{15}$ to $10^{17} \mathrm{~m}^{-3}$, and the electron temperature is expected range from 1 to $10 \mathrm{eV}[122,176]$.

Table 3.4: Tested plasma parameters for the triple probe experiments.

\begin{tabular}{ccc}
\hline Parameter & Range & Unit \\
\hline \hline Input RF Peak Amplitude Voltage & 175 to 350 & $\mathrm{~V}$ \\
DC Extraction Voltage & 700 to 2000 & $\mathrm{~V}$ \\
Internal Pressure & $0.75,1.00,1.25$ & Torr \\
Hollow Anode Radius & 0.3 & $\mathrm{~mm}$ \\
Ion Mean Free Path & $0.188,0.141,0.113$ & $\mathrm{~mm}$ \\
Knudsen Number for Ions & $1.25,0.94,0.75$ & unitless \\
\hline
\end{tabular}




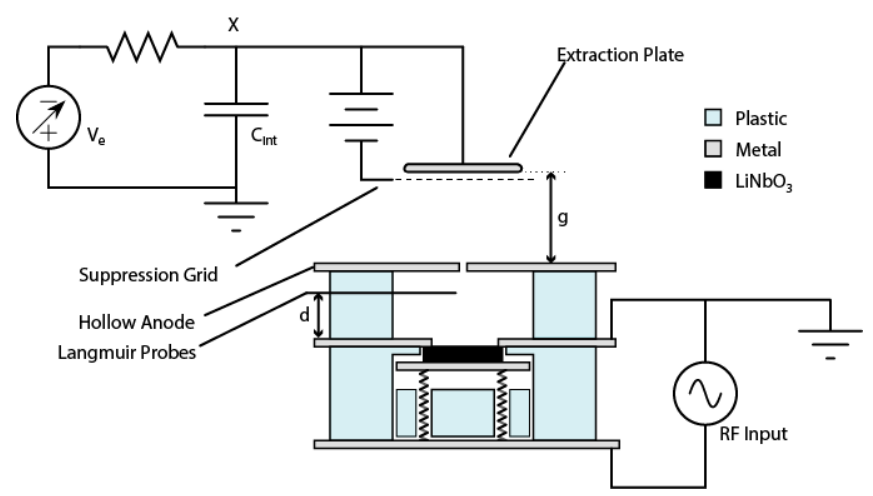

Figure 3.8: Cross section of the triple probe system used for electron temperature and density measurements.
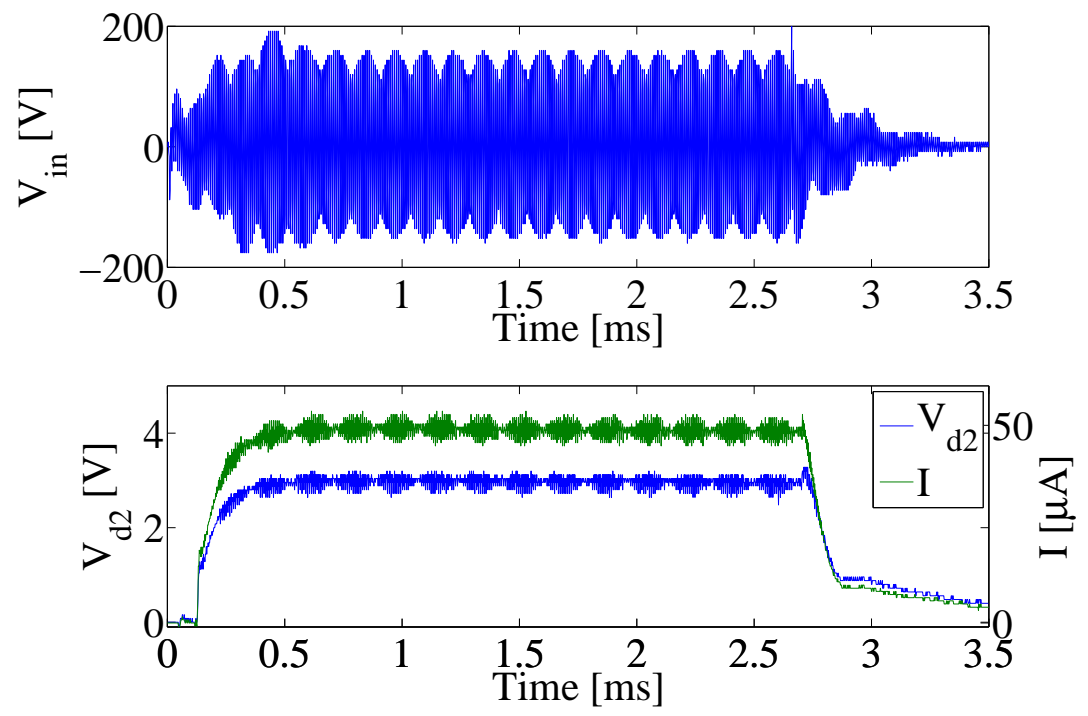

Figure 3.9: $\mathrm{RF}$ input voltage and $\mathrm{V}_{\mathrm{d} 2}$ (the voltage difference between the floating probe $\left(P_{2}\right)$ and the positively biased probe $\left.\left(P_{1}\right)\right)$ and I for an internal pressure of 1.25 Torr and $0.6 \mathrm{~mm}$ hollow anode. The probe measurements start $150 \mu \mathrm{s}$ into the pulse at approximately the same time as the input voltage reaches the peak value. 


\section{Chapter 4}

\section{Experimental Results}

This chapter shows the evaluation of the piezoelectric transformer plasma source (PTPS) as an ion source. The PTPS ion current was measured with respect to the ionizing gas type, extraction voltage, RF driving voltage, internal pressure, hollow anode radius, chamber depth, and operating time. The PTPS was also studied through COMSOL simulation to determine the influence of ion beam optics on the extracted ion beam and to ensure that a meniscus was formed by the extraction voltage and hollow anode.

Also presented in this chapter are the results of neutron generator testing and triple electrostatic probe testing. Neutron counts are shown for different $\mathrm{RF}$ driving voltages, internal pressures, pulse repetition rate, and extraction voltages. The electron temperature and density were measured through the triple electrostatic probe method with respect to the RF driving voltage, extraction voltage, and internal pressure.

Finally, the results of the PTPS experiments were compared with the ion current model presented in Chapter 2. Through a least-squares fit of the modeled current to the experimental data the electron density was approximated using an assumed electron temperature. The model was also used to show the impact of changing the electron current and density on the ion current extracted from the plasma. 


\subsection{Ion Source Characterization}

The PTPS was characterized and optimal operating parameters were determined by independently testing each parameter shown in Table 3.1. The following sections discuss the results for experiments on type of gas, input RF voltages, dc extraction voltages, hollow anode radii, and calculated internal pressures.

\subsubsection{Deuterium and Hydrogen Gas Testing}

The PTPS was tested using both deuterium and hydrogen to establish a functional relationship between the two gases and confirm the ion current dependence on ion mass. For this experiment, the extracted deuterium ions were assumed to be twice as massive as the extracted hydrogen ions. Figure 4.1 shows the ion current measured using a $400 \mathrm{~V}$ RF driving voltage, $0.3 \mathrm{~mm}$ hollow anode radius, 2.5 Torr internal pressure, and dc extraction voltage range of 200 to $2000 \mathrm{~V}$. The ion currents in Fig. 4.1 represent the average values for two extraction voltage sweeps for both hydrogen and deuterium gases. The ion current measured with the two gases followed the predicted trend of the more massive ion having a lower ion current. The ion current measured using deuterium gas was 17\% larger than predicted at the maximum extraction voltage, but had a functional relationship of $I_{D} \sim I_{H} / \sqrt{2}$, which is related to the Bohm criterion for ion extraction from a sheath [30,31]. The $I_{H} / \sqrt{2}$ function was also plotted in Fig. 4.1, further illustrating the functional relationship between deuterium and hydrogen ion current. This experiment permitted a comparison between the hydrogen and deuterium ion beams created by the PTPS. Based on the results of this experiment, all additional ion source characterization experiments were performed using hydrogen gas and measured currents were scaled by a multiplying factor of $1 / \sqrt{2}$. 


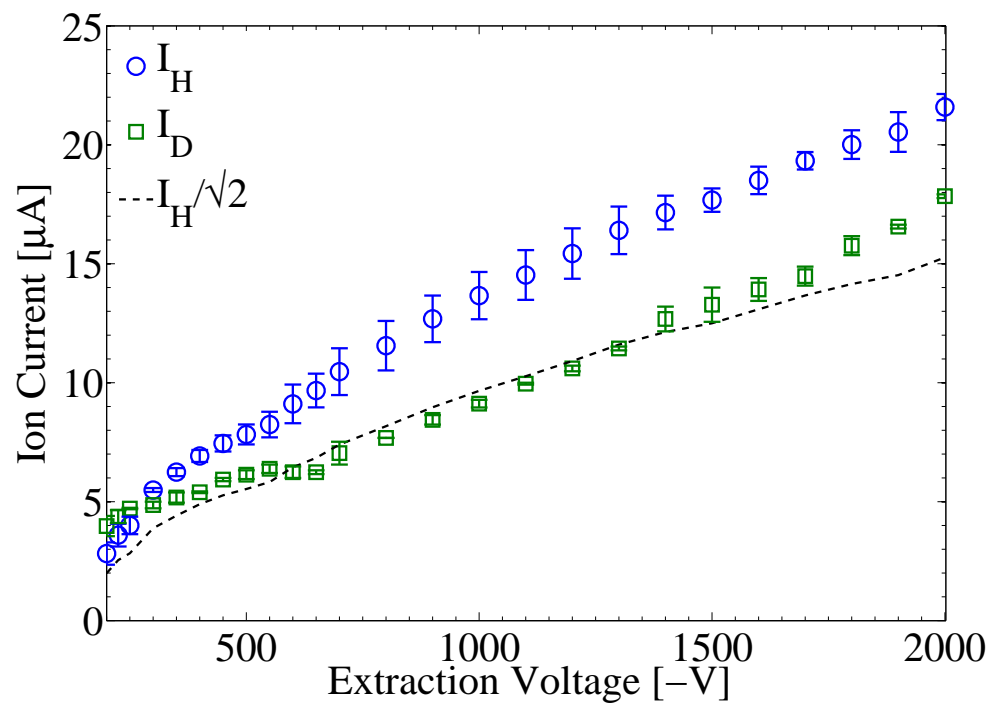

Figure 4.1: Average measured ion current with error bars representing the range for deuterium and hydrogen gases and $I_{H} / \sqrt{2}$ found using a $0.3 \mathrm{~mm}$ hollow anode radius, $400 \mathrm{~V}$ RF driving voltage, and 2.5 Torr internal pressure over a range of dc extraction voltages from 200 to $2000 \mathrm{~V}$.

\subsubsection{Extraction Voltage Testing}

The impact of the extraction voltage on the ion current was measured in two different experiments. In the first experiment, presented in 4.1.1, a $400 \mathrm{~V}$ RF driving voltage, $0.3 \mathrm{~mm}$ hollow anode radius, 2.5 Torr internal pressure, dc extraction voltage range of 200 to $2000 \mathrm{~V}$, and hydrogen and deuterium gases were used to show the impacts on gas type and extaction voltage on ion current. Doubling the extraction voltage from -1000 to $-2000 \mathrm{~V}$ resulted in 1.6 times greater currents for hydrogen (from 13.6 to $21.6 \mu \mathrm{A}$ ) and 1.96 the ion current for deuterium (from 9.1 to $17.8 \mu \mathrm{A}$ ). As is shown in Fig. 4.1, the deuterium ion current was greater than expected.

The second measurement was used to show that the space charge limit is reached. For this experiment, the PTPS was operated with RF driving voltages ranging from 200 to $400 \mathrm{~V}, 0.3 \mathrm{~mm}$ hollow anode radius, 2.0 Torr internal pressure 
over an extraction voltage magnitude ranging from 100 to $2000 \mathrm{~V}$, and hydrogen gas. The ion current for extraction voltage testing was plotted versus perveance to show the point at which the ion current reaches the space charge limited region (shown in Fig. 4.2). Perveance shows the space charge effect on the beam motion, thus the space charge limit is shown in Fig. 4.2 as the point wehere the curve rolls over. The ion current showed a nearly linear increase with extraction voltage magnitudes greater than 225, 400, and $550 \mathrm{~V}$ for 200,300 , and $400 \mathrm{~V}$ RF driving voltages, respectively.

Based on the ion current model discussed in Section 2.1 the ion current is porportional to the ratio of the extraction electric field and the electric field characteristic of the plasma. The ion currents shown in Figs. 4.1 and 4.2 show the linear trend of the ion current with increasing extraction voltage.

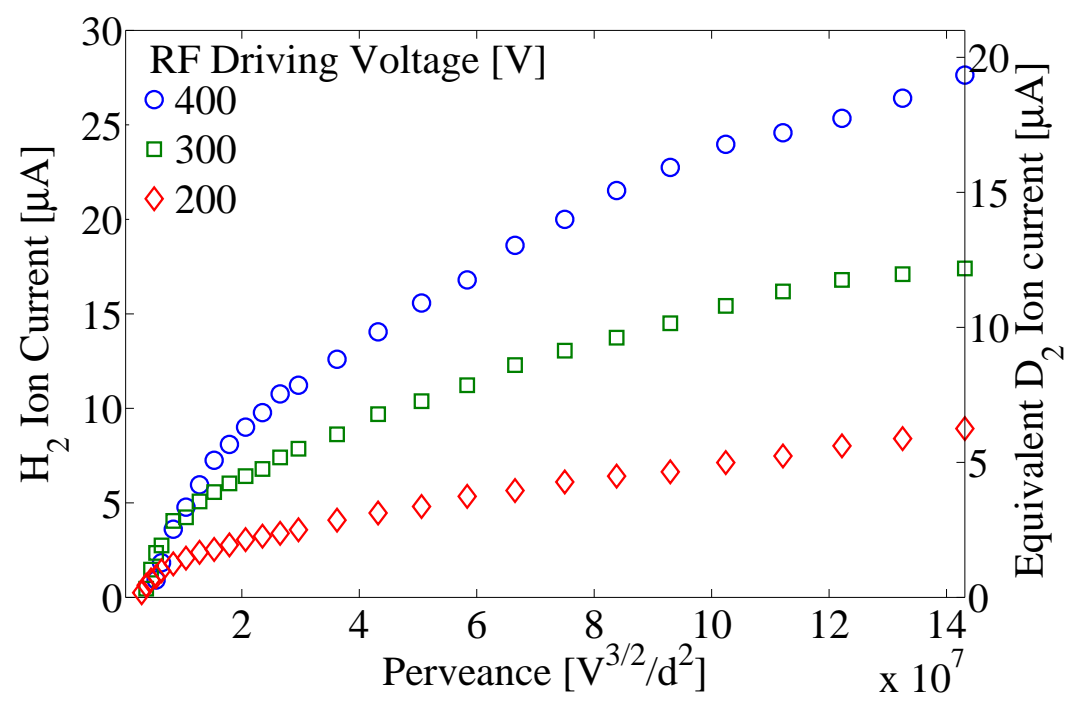

Figure 4.2: Measured hydrogen ion current for three RF driving voltages over a range of extraction voltages with a $0.3 \mathrm{~mm}$ hollow anode radius and an internal pressure of 2.0 Torr as well as equivalent deuterium ion current. 


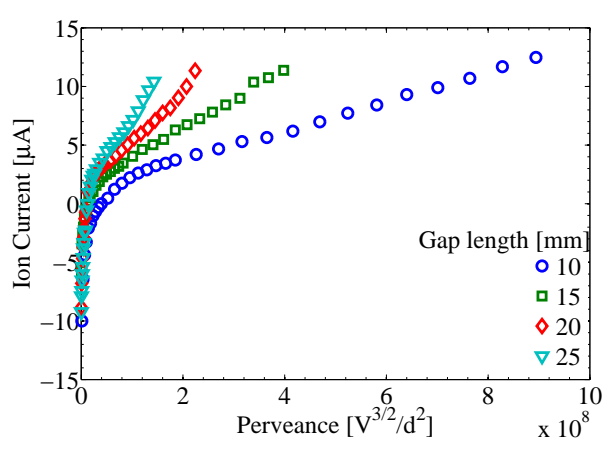

(a) 2.00 Torr Internal Pressure

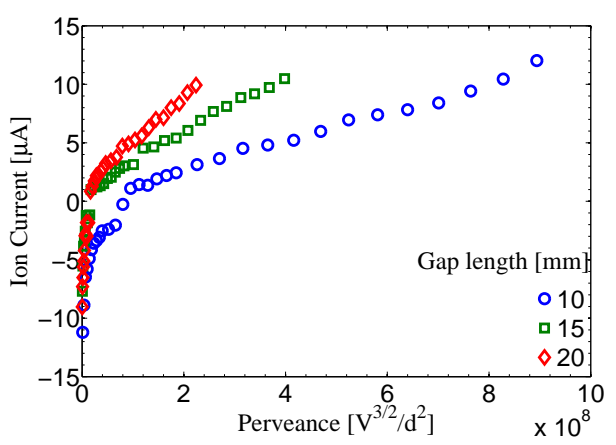

(b) 1.75 Torr Internal Pressure

Figure 4.3: Measured hydrogen ion current plotted versus perveance for gap distances ranging from 10 to $25 \mathrm{~mm}$ over a range of extraction voltages with a $0.3 \mathrm{~mm}$ hollow anode radius at 1.75 and 2.00 Torr internal pressures.

\subsubsection{Gap Distance Testing}

The impact of gap distance (the distance between the hollow anode and the extraction electrode) on ion current from the PTPS was tested at different internal pressures. Gap distances ranging from 10 to $25 \mathrm{~mm}$ were examined at 1.75 and 2.00 Torr internal pressure (corresponding to 17.9 and 20.5 sccm flow rates, respectively) with a $0.6 \mathrm{~mm}$ radius hollow anode, $300 \mathrm{~V} \mathrm{RF}$ driving voltage, extraction voltages from -100 to $-2000 \mathrm{~V}$, and hydrogen gas. The ion currents were plotted versus perveance to determine the space charge effect on the ion beam and relates to the convergence and divergence of the ion beam. Fig. 4.3 shows the ion currents at 2.00 and 1.75 Torr internal pressure for the various gap lengths and Fig. 4.4 shows each pressure at the same gap distance for comparison. For each gap length, the highest ion current was measured at the highest pressure, with an increase in ion current as perveance and extraction voltage increased. The space charge limit is reached at $-300 \mathrm{~V}$ extraction voltage. When comparing the gap distance, the same trend in ion current is observed for each internal pressure at the respective gap distance. 


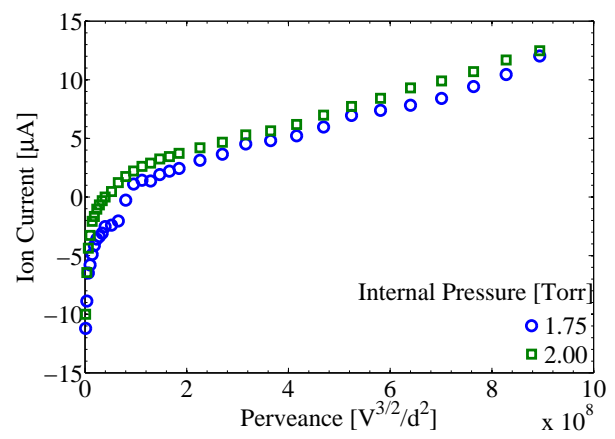

(a) $10 \mathrm{~mm}$

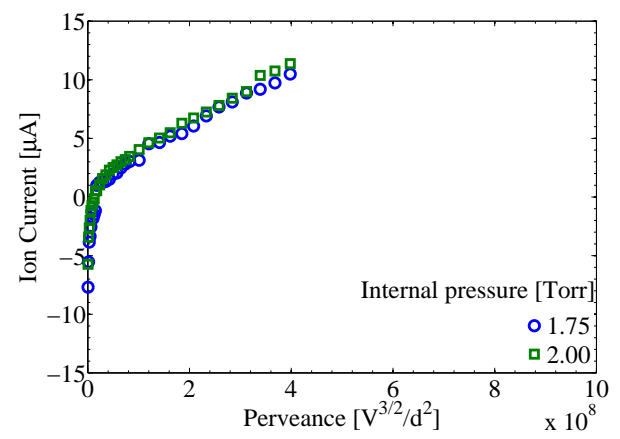

(b) $15 \mathrm{~mm}$

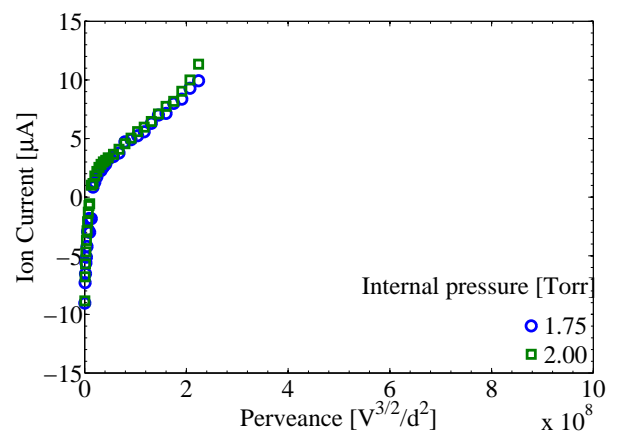

(c) $20 \mathrm{~mm}$

Figure 4.4: Measured hydrogen ion current plotted versus perveance for gap distances ranging from 10 to $20 \mathrm{~mm}$ over a range of extraction voltages with a $0.3 \mathrm{~mm}$ hollow anode radius at 1.75 and 2.00 Torr internal pressures. 


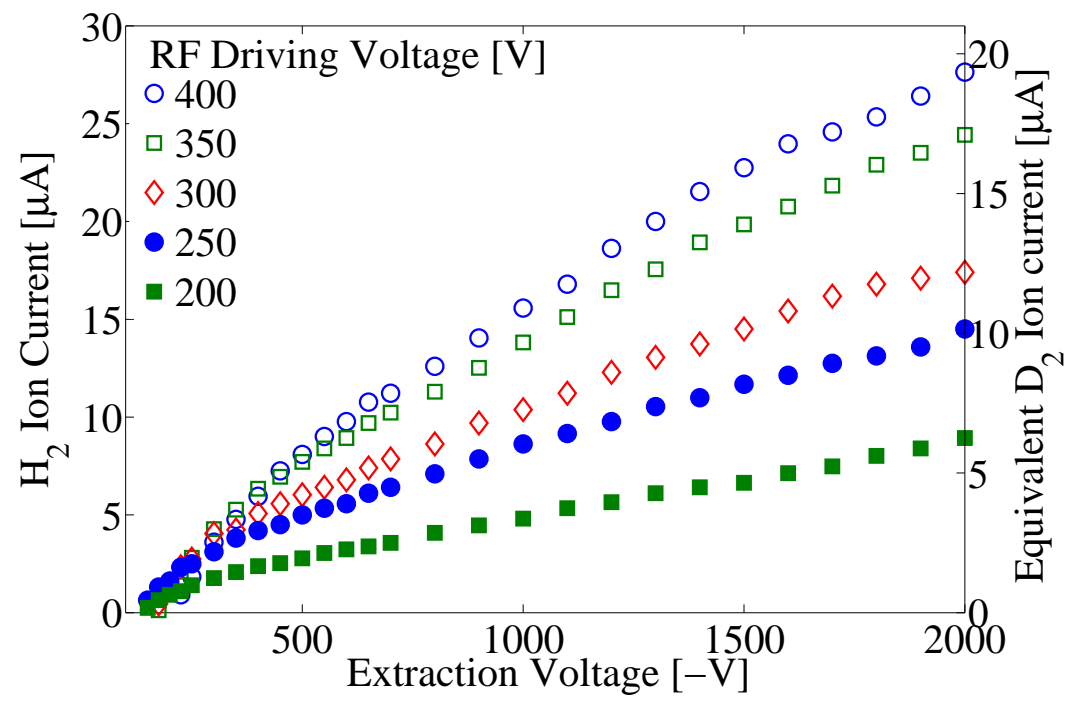

Figure 4.5: Measured hydrogen ion current for five RF driving voltages over a range of dc extraction voltages with a $0.3 \mathrm{~mm}$ hollow anode radius and an internal pressure of 2.0 Torr as well as equivalent deuterium ion current.

\subsubsection{RF Voltage Testing}

The PTPS was tested using different RF driving voltages to confirm the ion current dependence on plasma density. The impact of the RF driving voltage on the ion current was measured using a $0.3 \mathrm{~mm}$ hollow anode radius, 2.0 Torr internal pressure, and 100 to $2000 \mathrm{~V}$ extraction voltage, over a range of RF driving voltages from 200 to $400 \mathrm{~V}$. The ion currents are shown in relation to the extraction voltage in Fig. 4.5. Doubling the RF driving voltage from 200 to $400 \mathrm{~V}$ resulted in more than triple the measured ion current (from 9.0 to $27.8 \mu \mathrm{A}$ ). These measurements show a linear proportional relationship between the plasma density, tested through the RF driving voltage, and the ion current. Based on the results of this experiment, subsequent tests used the RF driving voltage of $400 \mathrm{~V}$. 


\subsubsection{Hollow Anode Radius Testing}

The impact of the hollow anode size on the ion current was measured using a $400 \mathrm{~V}$ RF driving voltage, 1.5 and 3.0 Torr internal pressures, 100 to $2000 \mathrm{~V}$ magnitude extraction voltages, and 0.15 and $0.3 \mathrm{~mm}$ hollow anode radii. Figure 4.6 shows the ion currents measured using the 0.3 and $0.15 \mathrm{~mm}$ radius hollow anode. Doubling the size of the radius from 0.15 to $0.3 \mathrm{~mm}$ resulted in approximately 15 times the ion current; at 3 Torr internal pressure and $-2000 \mathrm{~V}$ extraction, the current was $1.6 \mu \mathrm{A}$ for the $0.15 \mathrm{~mm}$ radius and $25.0 \mu \mathrm{A}$ for the $0.3 \mathrm{~mm}$ radius. These measurements show that the hollow anode size has a significant impact on the ion current. Based on (3.1), larger hollow anode sizes require a higher flow rate to achieve the same pressures. Flow rates and corresponding internal pressures for this experiment are shown in Table 4.1. Due to limitations of the mass flow controller used in the system, the $0.3 \mathrm{~mm}$ radius hollow anode was the largest tested in this experiment.

The current model described in Section 2.1 predicts that the ion current is directly proportional to the hollow anode radius size. Based on the model, the doubling the size of the ion current should result in four times the ion current. Based on the dashed and dotted lines in Fig. 4.6 representing the measured ion currents for the $0.15 \mathrm{~mm}$ hollow anode radius multiplied by four, the measured ion currents for the $0.3 \mathrm{~mm}$ hollow anode radius were approximately four times larger than predicted using the model. This shows a possible breakdown in the scaling of the predictive model.

\subsubsection{Internal Pressure Testing}

The internal pressure of the PTPS was a function of the flow rate and the hollow anode radius. Gas in the vacuum background could provide conditions suitable for field ionization from the extraction electrode, or diffuse discharges in the vacuum background within the vicinity of the extraction plate, leading to 
Table 4.1: Gas flow rate and calculated internal pressure for 0.15 and $0.3 \mathrm{~mm}$ hollow anode aperture.

\begin{tabular}{ccc}
\hline $\mathrm{a}[\mathrm{mm}]$ & $\mathrm{Q}[\mathrm{sccm}]$ & $\mathrm{P}$ [Torr] \\
\hline \hline 0.15 & 3.8 & 1.5 \\
0.15 & 7.7 & 3.0 \\
0.3 & 15.4 & 1.5 \\
0.3 & 20.4 & 3.0 \\
\hline
\end{tabular}

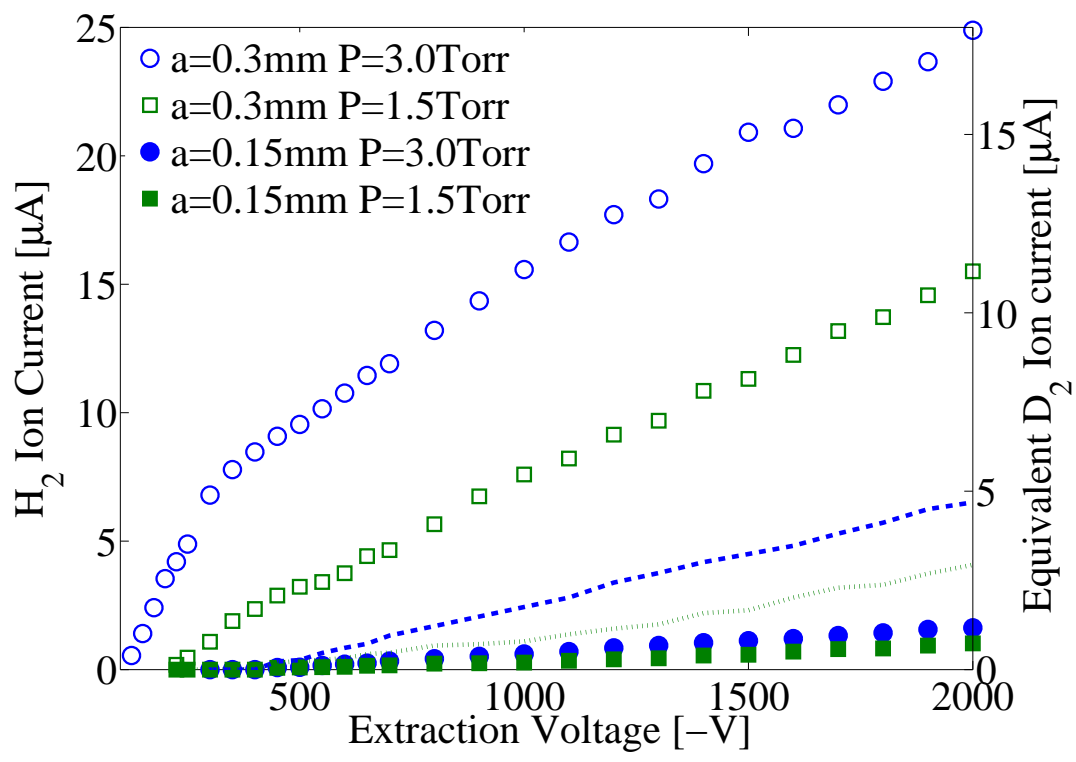

Figure 4.6: Measured ion current for $400 \mathrm{~V}$ RF driving voltage over a range of dc extraction voltages for $0.15 \mathrm{~mm}$ (filled) and $0.3 \mathrm{~mm}$ (open) hollow anode radii and internal pressures of 1.5 Torr (squares) and 3.0 Torr (circles) as well as equivalent deuterium ion current. The predicted current based on the ratio of the hollow anode radius size is plotted based on the smaller aperture data for the 3.0 Torr (blue dashed line) and 1.5 Torr (green dotted line). 
inaccurate measurements of the ion currents from the PTPS. To achieve the ion current goal of the PTPS while keeping the gas in the vacuum background at a minimum, a high degree of ionization was required.

The impact of internal pressure on the ion current was measured using two different experiments. In the first experiment, the PTPS was operated with a $400 \mathrm{~V}$ RF driving voltage, $0.3 \mathrm{~mm}$ hollow anode radius, 100 to $2000 \mathrm{~V}$ magnitude extraction voltage, and 1.5 and 3.0 Torr internal pressures. Changes in the internal pressure were achieved by varying the flow rate resulting in internal pressures of 1.5 and 3.0 Torr, as shown in Table 4.1. The results of this experiment are shown in Fig. 4.6. Doubling the internal pressure from 1.5 to 3.0 Torr resulted in a 1.6 times increase in current (from 15.5 to $25.0 \mu \mathrm{A}$ for the $0.3 \mathrm{~mm}$ hollow anode radius). Due to the capacity of the flow controller, testing at higher internal pressures required a smaller hollow anode radius.

The impact of PTPS internal pressure on ion current was tested using a $0.15 \mathrm{~mm}$ hollow anode and two different crystals. The crystals were both operated at a driving voltage of $400 \mathrm{~V}$ with internal pressures ranging from 1.5 to 11.0 Torr and ion extraction voltage of $-2000 \mathrm{~V}$. The flow rates required for the various internal pressures are shown in Table 4.2. Fig. 4.7 shows that three internal pressure sweeps were done for each crystal. Crystal \#56 was from the April 2014 batch of crystals and was new at the time of testing, and crystal \#4 was from the April 2013 batch of crystals and had been used for several different tests. Both crystals had freshly painted silver electrodes applied to the piezoelectric crystal at the start of this testing. Crystal \#4 only operated consistently at internal pressures up to 7.5 Torr, while crystal \#56 operated up to 11.0 Torr with both crystals showing an increase in ion current with internal pressure. Fig. 4.8 shows the average of the three tests shown in Fig. 4.7 with error bars showing the range of currents. These graphs also show little variation in ion currents measured with different piezoelectric transformers in different trials. 
Table 4.2: Gas flow rate and calculated internal pressure for $0.15 \mathrm{~mm}$ hollow anode aperture

\begin{tabular}{cc}
\hline $\mathrm{Q}$ [sccm] & $\mathrm{P}$ [Torr] \\
\hline \hline 3.8 & 1.5 \\
5.1 & 2.0 \\
6.4 & 2.5 \\
7.7 & 3.0 \\
8.9 & 3.5 \\
10.2 & 4.0 \\
11.5 & 4.5 \\
12.8 & 5.0 \\
14.1 & 5.5 \\
15.4 & 6.0 \\
16.6 & 6.5 \\
18.2 & 7.0 \\
19.2 & 7.5 \\
20.4 & 8.0 \\
21.8 & 8.5 \\
23.1 & 9.0 \\
24.4 & 9.5 \\
25.8 & 10.0 \\
26.8 & 10.5 \\
28.2 & 11.0 \\
\hline
\end{tabular}




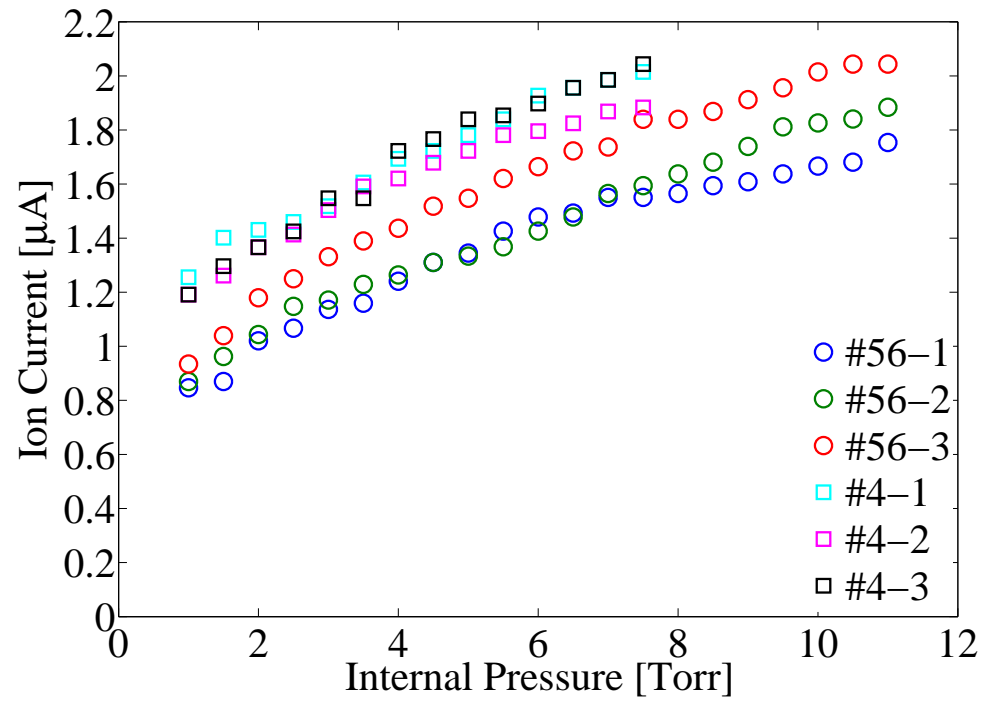

Figure 4.7: Measured hydrogen ion current found using a $0.15 \mathrm{~mm}$ hollow anode radius, $400 \mathrm{~V}$ RF driving voltage, and $-2000 \mathrm{~V}$ dc extraction voltage over a range of pressures from 1.5 to 11.0 Torr for two different piezoelectric transformers. Each piezoelectric transformer $(\# 4$ and \#56) was operated with identical parameters for three trials $(1,2$, and 3$)$ 


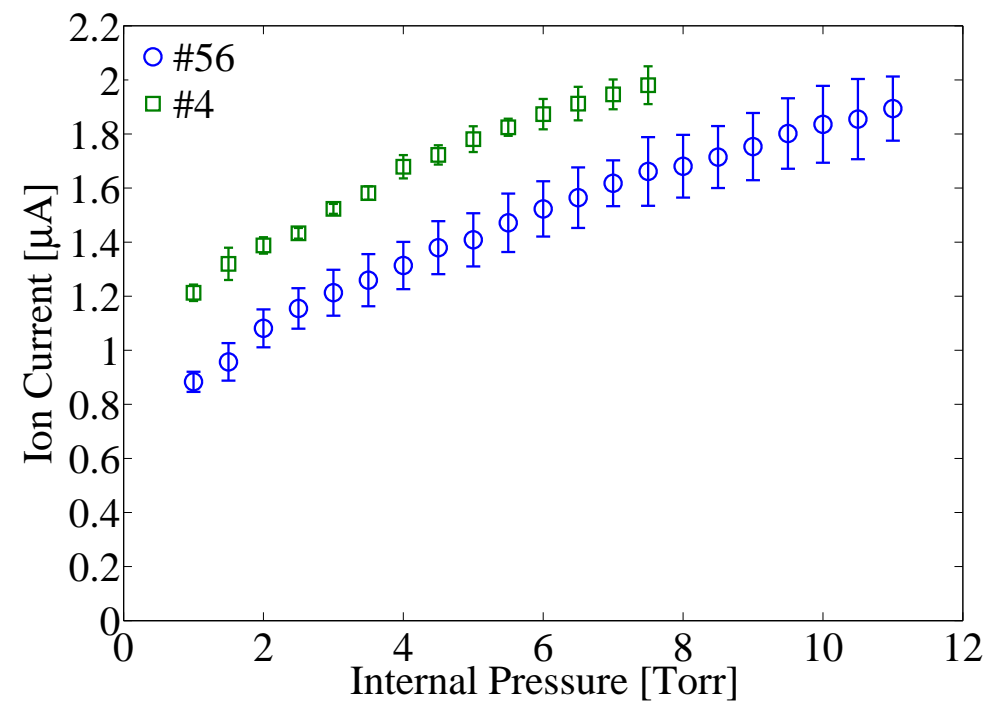

Figure 4.8: Averaged measured hydrogen ion current found using a $0.15 \mathrm{~mm}$ hollow anode radius, $400 \mathrm{~V}$ RF driving voltage, and -2000 V dc extraction voltage over a range of pressures from 1.5 to 11.0 Torr for two different piezoelectric transformers.

\subsubsection{Holder Depth Testing}

Holders with different depths were tested to determine if the size of the plasma generating region had an impact on the ion current collected. The different depths of the holders allowed for a different plasma generation area between the piezoelectric transformer surface and the hollow anode. Holders with depths of 3, 6, and $9 \mathrm{~mm}$ were used for this experiment. Fig. 4.9 shows the ion currents measured over a range of dc extraction voltages with a $0.3 \mathrm{~mm}$ hollow anode radius, an $\mathrm{RF}$ driving voltage of $350 \mathrm{~V}$, and an internal pressure of 1.25 Torr with chamber depths of 3,6 , and $9 \mathrm{~mm}$. At the maximum extraction voltage, the measured hydrogen ion currents were 13.7, 8.8, and $6.9 \mu \mathrm{A}$ for the holders with 3, 6, and $9 \mathrm{~mm}$ depths, respectively. 


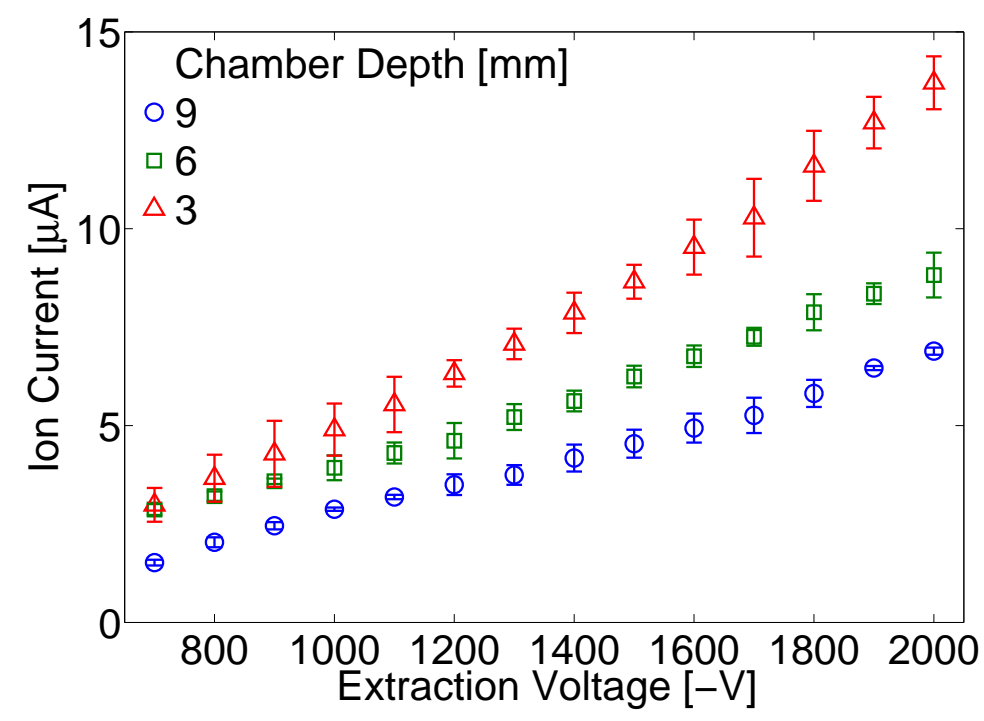

Figure 4.9: Measured hydrogen ion current for three different chamber depths over a range of dc extraction voltages with a $0.3 \mathrm{~mm}$ hollow anode radius, an $\mathrm{RF}$ driving voltage of $350 \mathrm{~V}$, and an internal pressure of 1.25 Torr.

\subsubsection{Operational Time Testing}

To determine the affect of operational time on ion current extracted from the PTPS, the experiment was performed with the same input parameters for extended periods of time. Fig. 4.10 shows the normalized ion current with respect to time for pulsed mode operation for time periods ranging from 4 to 10 hours, with the open markers corresponding to the first crystal and the closed markers corresponding to the second crystals. Two different cystals were tested to show that each crystal required a similar warm up time and operated similarly over time. Based on these results, it was determined that the PTPS should be operated for at least 45 minutes before data are taken, because of the decrease to approximately $50 \%$ of the initial measured ion current. The high initial current could be due to contamination on the piezoelectric transformer or PTPS holder caused by the device being out of vacuum for any period of time resulting in ions other than those from the hydrogen plasma. This experiment showed that the 


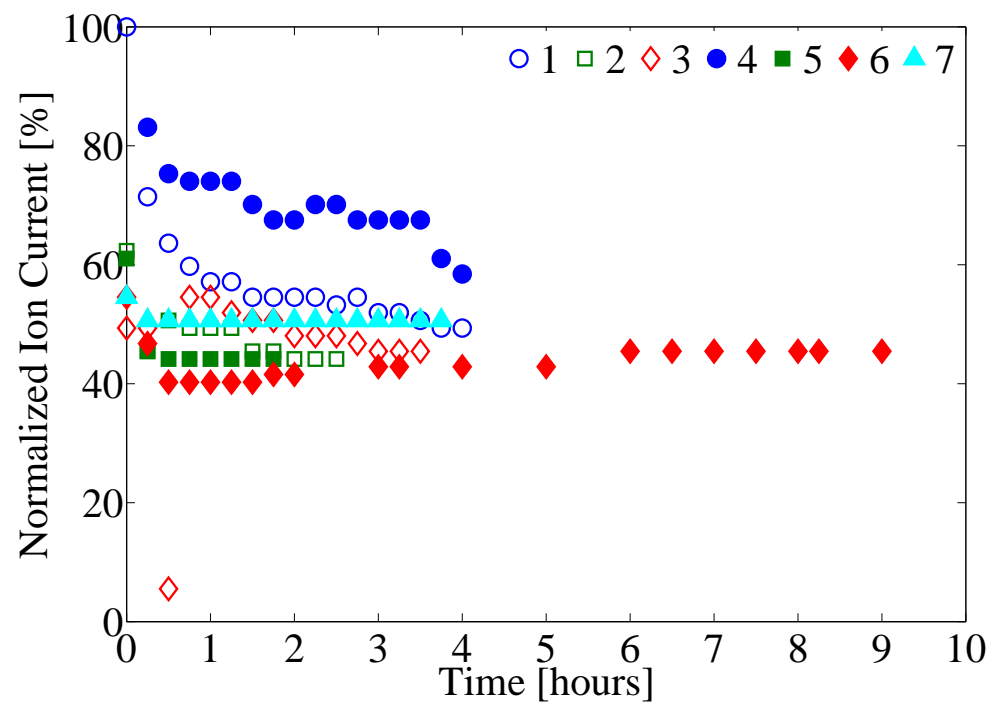

Figure 4.10: Ion current normalized to the initial ion current over time for two different crystals, with the open markers representing crystal one and the filled markers representing crystal two.

PTPS was capable of operating for extended periods of time.

\subsubsection{Ion Optic Simulation}

One method of improving the performance of an ion source is to improve the focus of the ions being extracted from the hollow anode aperture. As discussed in Section 2.1, the ions are extracted from the plasma meniscus, the shape of which is controlled by the extraction electric field and the hollow anode. The ion beam shape is controlled by the shape of the meniscus, with well collimated beams coming from a convex meniscus $[69,70,72,74]$. Through COMSOL simulations, shown in Fig. 4.11 for the 0.15 and $0.3 \mathrm{~mm}$ radius hollow anode, it was determined that the PTPS meniscus was concave for extraction voltages ranging from 100 to 2000 V [183]. Simulations were used rather than experiments because the aim was to merely show the proof of principle that an ion optic should improve the ion current yield. 


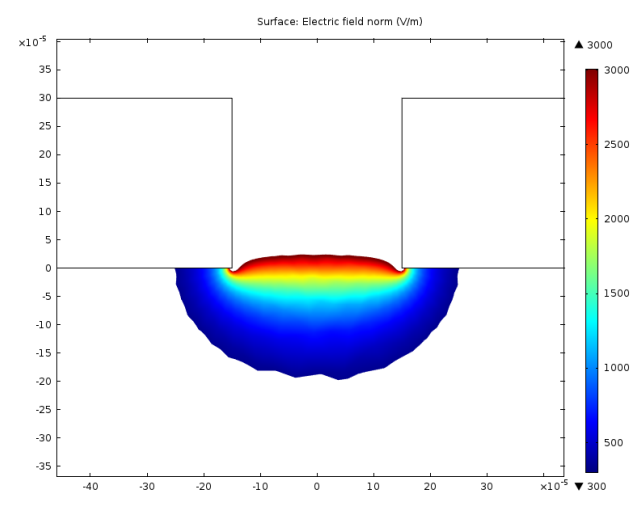

(a) $0.15 \mathrm{~mm}$ hollow anode radius

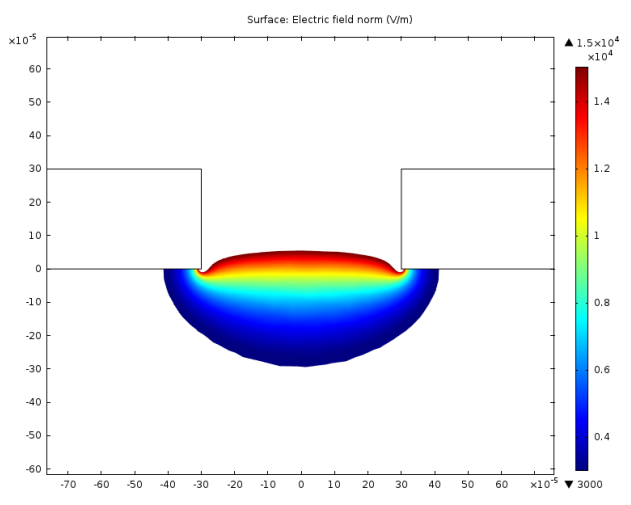

(b) $0.3 \mathrm{~mm}$ hollow anode radius

Figure 4.11: COMSOL simulation of meniscus formed inside the hollow anode aperture at $2000 \mathrm{~V}$ extraction.

To further collimate the ion beam, an optic could be added between the hollow anode and the extraction plate of the experiment. As is shown in Fig. 4.12, the ion beam collimates when an optic is added to the extraction system. The simulation shows that to be effective, the optic should be at least $3 \mathrm{~mm}$ from the hollow anode. The simulation also shows that if a large extraction plate is used, most ions will be collected by it. An optic would be useful in cases where a large extraction plate is not feasible, such as in a compact neutron generator that uses a small deuterium-doped target, because a greater percentage of the ions would reach the target.

\subsubsection{Summary of Ion Source Characterization}

The PTPS ion current was characterized and optimized through RF input driving voltage, dc extraction voltage, hollow anode size, internal pressure, and gas type. The PTPS was tested using deuterium and hydrogen gas to show a functional relationship between the two gases of $I_{D} \sim I_{H} / \sqrt{2}$. Further 


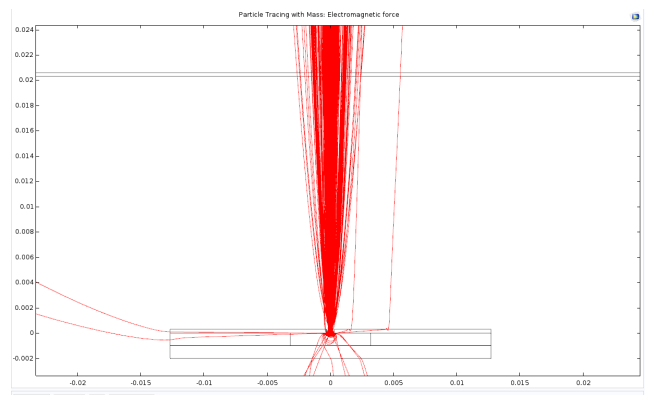

(a) No Optic

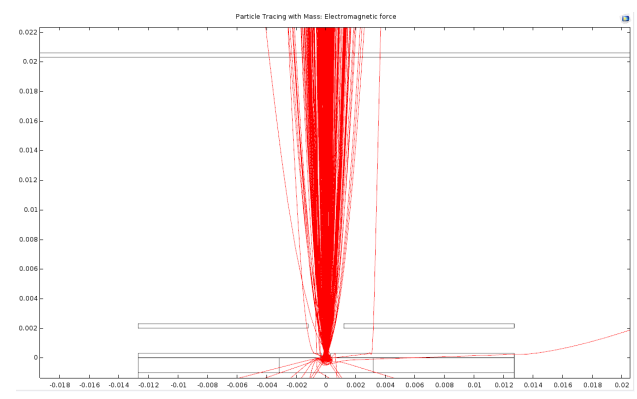

(c) $2 \mathrm{~mm}$

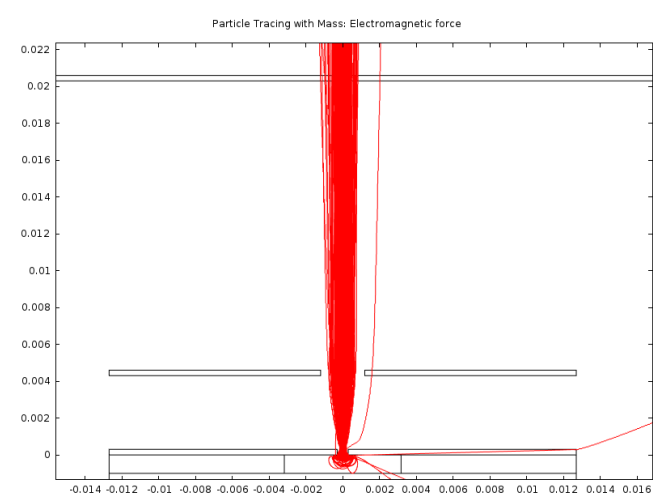

(e) $4 \mathrm{~mm}$

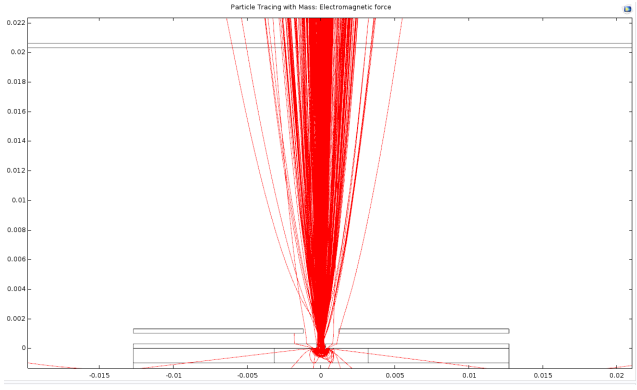

(b) $1 \mathrm{~mm}$

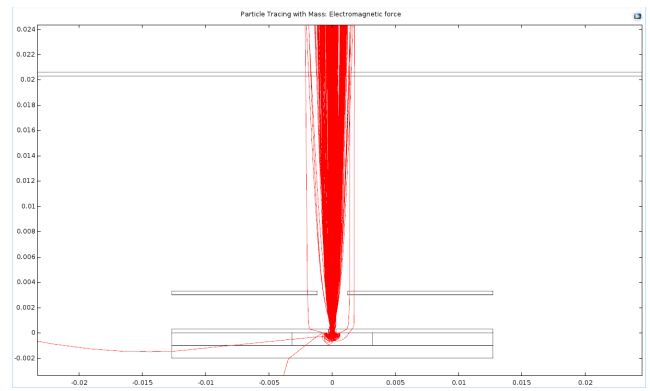

(d) $3 \mathrm{~mm}$

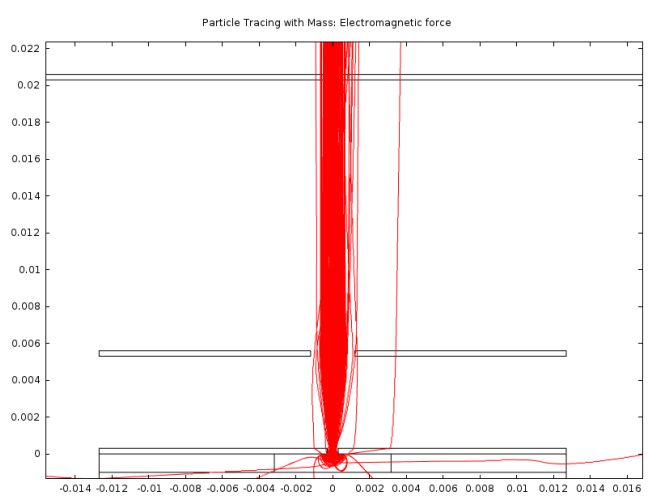

(f) $5 \mathrm{~mm}$

Figure 4.12: COMSOL simulation of the PTPS with a $2.4 \mathrm{~mm}$ opening optic at $-100 \mathrm{~V}$ at different spacing from the $0.3 \mathrm{~mm}$ radius hollow anode with the $-1000 \mathrm{~V}$ extraction voltage. 
characterization showed that the hollow anode size had the greatest impact on the ion currents, while the RF driving voltage had a greater influence on plasma density than the internal pressure. The extraction voltage also proved to be a means of increasing the ion current output of the PTPS, and holders with the smallest depths produced the largest ion currents. Using different chamber depths showed that increasing the chamber depth, and as such the spacing between the grounded hollow anode and the powered piezoelectric transformer, resulted in a decrease in ion current. The PTPS was operated using the same parameters for an extended period of time, showing that the PTPS should be operated for at least 45 minutes to reach a stable current, but was capable of operating for 10 hours. Finally, through simulation it was shown that a meniscus was formed by the extraction voltage and the hollow anode and the addition of an ion optic would result in a more collimated ion beam.

\subsection{Neutron Generation Testing}

The PTPS-DC experiment was used to show that the PTPS was capable of producing sufficient ion beam currents to generate neutrons with a dc accelerating voltage. Using a series of one hour tests described in Section 3.3, the PTPS-DC system was used to determine what input parameters had the greatest impact on the neutrons detected. Fig. 4.13 shows the results of the boxcar averaged PTPSDC experiment. The results were boxcar averaged to segment each hour into ten minute intervals $(\mathrm{n}=26)$. The $5 \%$ false alarm probability (FAP) and average background are also shown in Fig. 4.13. Counts above these levels indicate that neutrons were present.

The PTPS was tested with five different accelerating voltages ranging from 57 to $105 \mathrm{kV}$. At accelerating voltages greater than $69 \mathrm{kV}$, count rates above background were observed with a significance of p less than 0.05. The PTPSDC experiment was also tested to determine the impact of the PTPS internal 
Table 4.3: Average counts and false alarm probability for the PTPS-DC test.

\begin{tabular}{ccc}
\hline Accelerating Potential $[\mathrm{kV}]$ & Average Count Rate $\left[10 \mathrm{~min}^{-1}\right]$ & False Alarm Probability \\
\hline \hline 57 & 97.4 & $12 \%$ \\
69 & 110.5 & $4.3 \%$ \\
82 & 135.7 & $0.07 \%$ \\
92 & 166.2 & $\sim 0.0 \%$ \\
105 & 220.9 & $\sim 0.0 \%$ \\
\hline
\end{tabular}

pressure, RF driving voltage, and repetition rate. No significant influence of these parameters was observed, as is shown in Fig. 4.13. Fewer input parameters were tested with the 92 and $105 \mathrm{kV}$ accelerating voltages due to breakdown in the vacuum chamber.

The average count rates were calculated for each accelerating potential, and are shown in Table 4.3 with the corresponding FAP. The data were presented in a box and whisker plot to show the distribution for each test. The blue box shows the first and third quartiles of the data, the black whiskers show the maximum and minimum values, the red line inside the box shows the median value of the data set, and any additional red dots outside the boxes represent the outliers for the data. When the accelerating potential was increased from 57 to $105 \mathrm{kV}$, the average count rate increased from 97.5 to 220.9 counts/10 min. The FAP also decreased from $12 \%$ to less than $0.1 \%$ over the same range of accelerating potentials, with accelerating potentials greater than $82 \mathrm{kV}$ having a FAP of less than $0.1 \%$. This test demonstrated that the PTPS was capable of producing sufficient ion currents for neutron counts above background when coupled with a deuterium-doped target powered by a dc accelerating potential.

\subsection{Electron Temperature and Density Estimates}

The PTPS plasma was characterized using the plasma electron density, electron temperature, and extracted ion current. This characterization was 


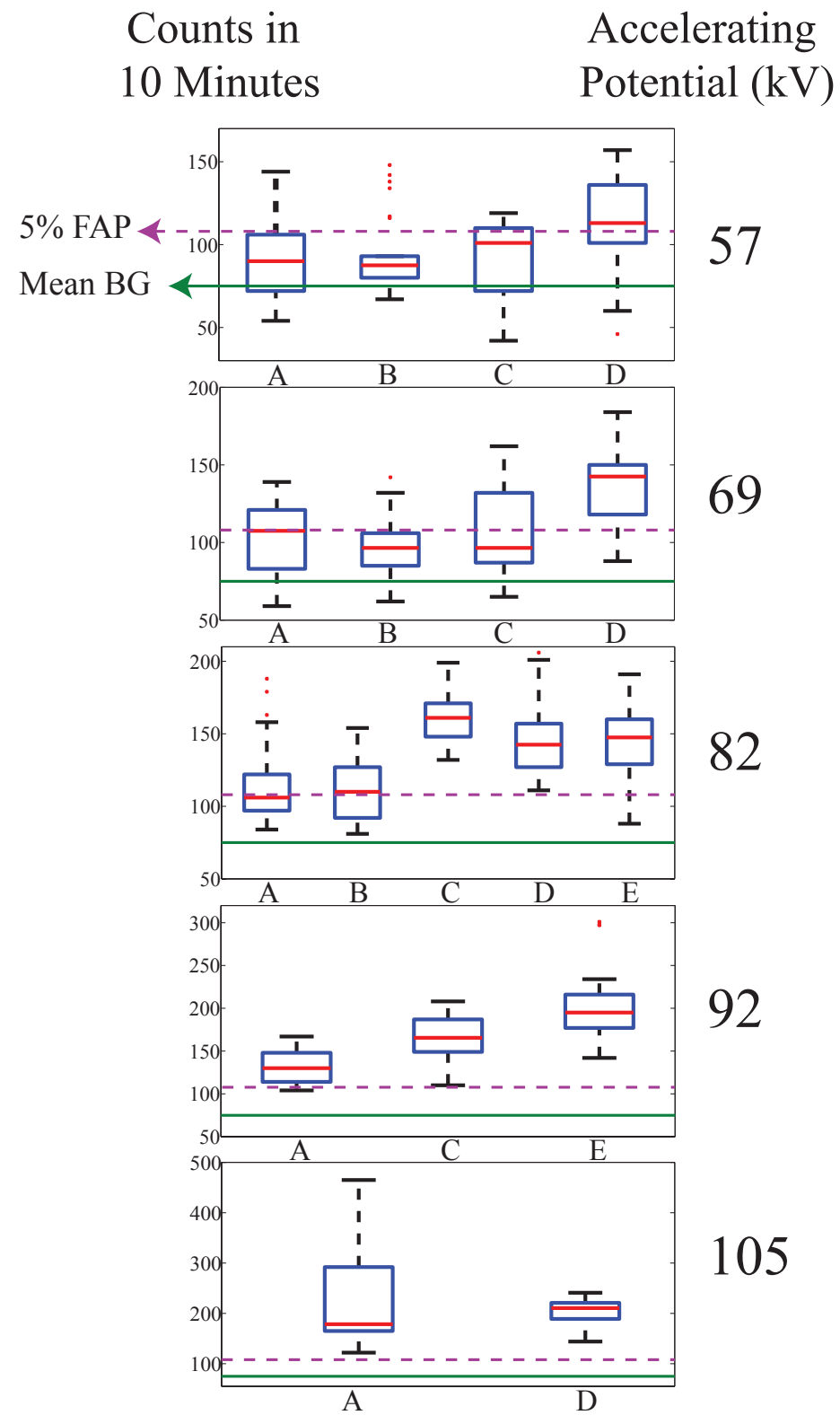

Figure 4.13: Neutron Counts in 10 minutes as a function of accelerating potential for the test matrix shown ain Table 3.2. In these plots the BG is the background neutron count and the FAP is the false alarm probability. 
accomplished through the use of a range of extraction voltages, $R F$ input voltages, and internal pressures using the system described in Section 3.4. The experiments were divided into two sets. The first set of experiments was used to confirm that the extraction voltage did not affect the fundamental plasma characteristics with a constant RF input voltage and internal pressure. The second set of experiments was used to determine whether the RF input voltage and internal pressure of the PTPS affected the electron temperature and density of the plasma. The ion current was measured as a reference for both experiments. Each experiment was performed three times under identical conditions and each data point represents the average of the results from the three tests with the error bars showing the range of each measurement.

Increasing the extraction voltage was shown to result in an increase in ion current in Subsection 4.1.2. To confirm that the extraction voltage only affected the ion current and not the electron temperature and density of the plasma, the electron temperature and density of the plasma and the ion current were monitored while the extraction voltage was swept from -700 to $-2000 \mathrm{~V}$.

Fig. 4.14 shows the electron temperature as it relates to the extraction voltage. The electron temperature calculated using (2.9) was determined to be approximately $4.45 \mathrm{eV}$. The electron temperature remained constant over the range of extraction voltages tested.

The ion current model allowed for an order of magnitude approximation once the electron temperature and ion current were known. The ion mass used for the calculation was that of $\mathrm{H}_{3}{ }^{+}$because a plasma generated in the 1 Torr pressure range would have $\mathrm{H}_{3}{ }^{+}$as the dominant ion as described in Section 2.3. Ion currents were extracted from a $0.6 \mathrm{~mm}$-deep holder (which represents the same distance between the electrostatic probes from the piezoelectric transformer surface). The electron density of the plasma was approximated using a non-linear least squares fit with the measured ion currents (shown in Fig. 4.15), average 


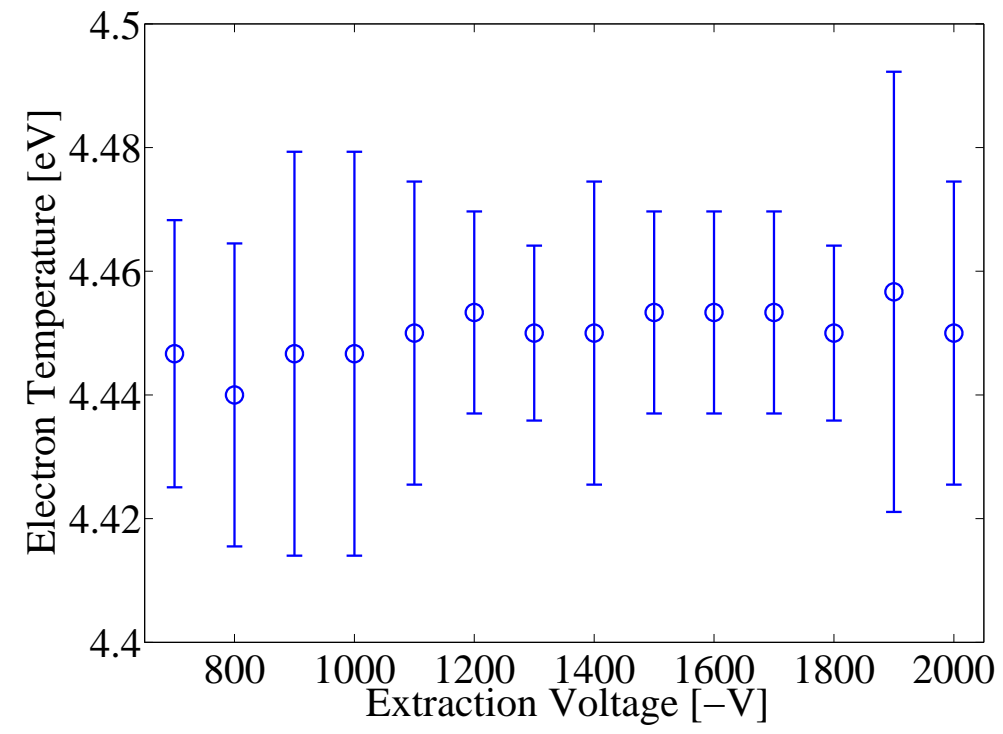

Figure 4.14: Measured electron temperature of the plasma over a range of dc extraction voltages with a $0.3 \mathrm{~mm}$ hollow anode radius, an $\mathrm{RF}$ driving voltage of $350 \mathrm{~V}$, and an internal pressure of 1.25 Torr.

electron temperature of $4.45 \mathrm{eV}$ (calculated from Fig. 4.14), and the ion current model in (2.1). The electron density was estimated to be $2.3 \times 10^{15} \mathrm{~m}^{-3}$ for a $\mathrm{H}_{3}{ }^{+}$plasma as shown in Fig. 4.15 with the measured ion current for comparison.

The electron density was initially estimated using the orbital motion limited (OML) approach using (2.10), with the measured electron temperature and assuming $\mathrm{H}_{3}{ }^{+}$ion mass as shown in Fig. 4.16. This resulted in an average estimated electron density of $5.6 \times 10^{16} \mathrm{~m}^{-3}$, an order of magnitude greater than the $10^{15} \mathrm{~m}^{-3}$ predicted using the ion current model. Based on the ion trajectories described in Chapter 2, this increase was most likely attributable to the destruction of OML as well as elastic scattering in the sheath surrounding the probe. While an order of magnitude above the expected value, the uncorrected plasma electron density shows little change over the range of extraction voltages tested.

Fig. 4.17 shows the electron density calculated using the measured electron 


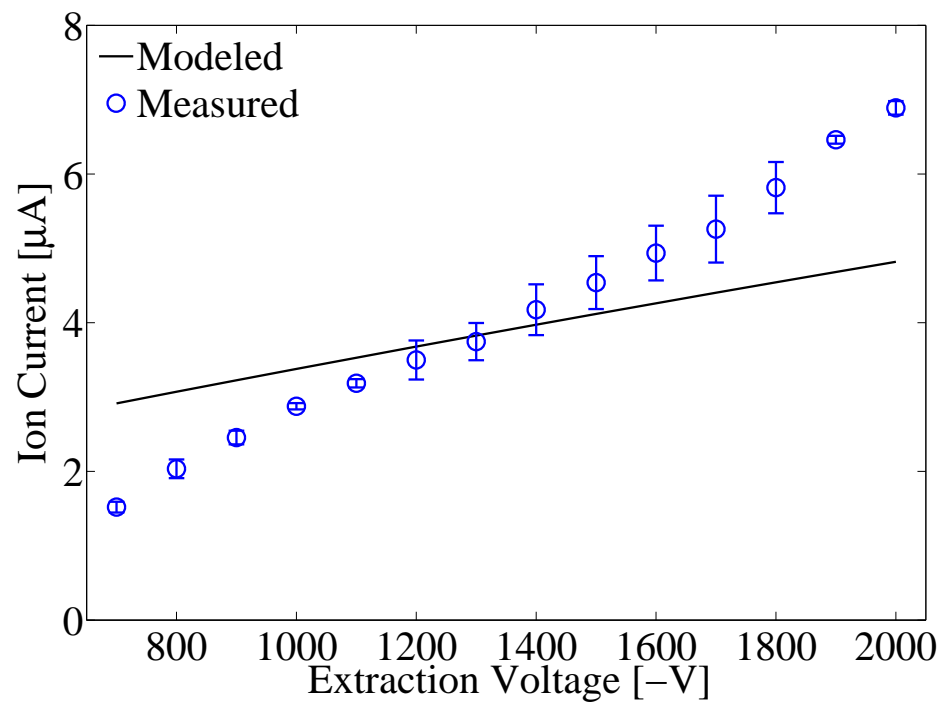

Figure 4.15: Measured ion current over a range of dc extraction voltages from 700 to $2000 \mathrm{~V}$ with a $0.3 \mathrm{~mm}$ hollow anode radius, an RF input voltage of $350 \mathrm{~V}$, and an internal pressure of 1.25 Torr plotted with ion current calculated using (2.1) for $\mathrm{H}_{3}{ }^{+}$ions at an electron temperature of $4.45 \mathrm{eV}$ and density of $2.5 \times 10^{15} \mathrm{~m}^{-3}$. 


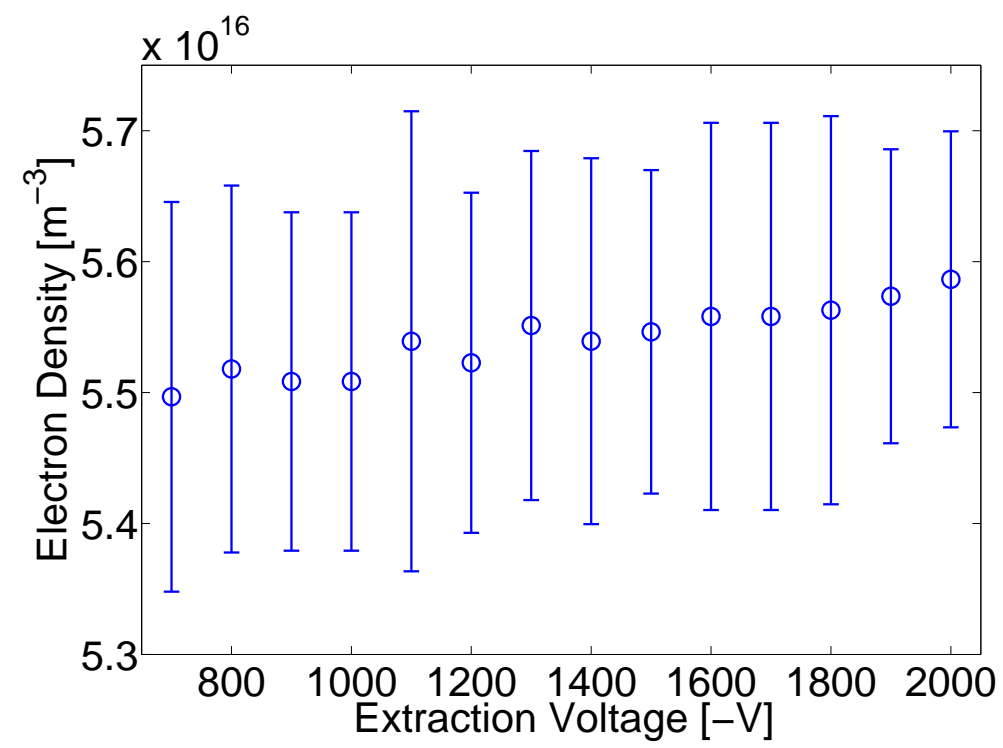

Figure 4.16: Uncorrected estimated electron density assuming $\mathrm{H}_{3}{ }^{+}$ions over a range of dc extraction voltages with a $0.3 \mathrm{~mm}$ hollow anode radius, and RF driving voltage of $350 \mathrm{~V}$, and an internal pressure of 1.25 Torr.

temperature and ion saturation current from the triple electrostatic probe experiment with a $\mathrm{H}_{3}{ }^{+}$plasma ion. The electron density in Fig. 4.17 was corrected to account for errors in the ion current measurement caused by collisions and scattering in the sheath as described in Section 3.4. After applying the correction factor, the average electron density was $6.6 \times 10^{15} \mathrm{~m}^{-3}$. The corrected electron density value falls within the predicted order of magnitude for the ion current and temperature found in this experiment (as shown in Table 4.4) and remained constant over the tested range of extraction voltages.

Fig. 4.18 shows the ion current measured with a constant $-2000 \mathrm{~V}$ extraction voltage, RF input voltages ranging from 175 to $350 \mathrm{~V}$, and internal pressures ranging from 0.75 to 1.25 Torr to allow for a comparison of the ion current and the electron temperature and density. Ion currents ranged from 1.89 to $6.93 \mu \mathrm{A}$, and increased with the RF input voltage. The highest ion currents were measured at the highest RF input voltage and internal pressure. 


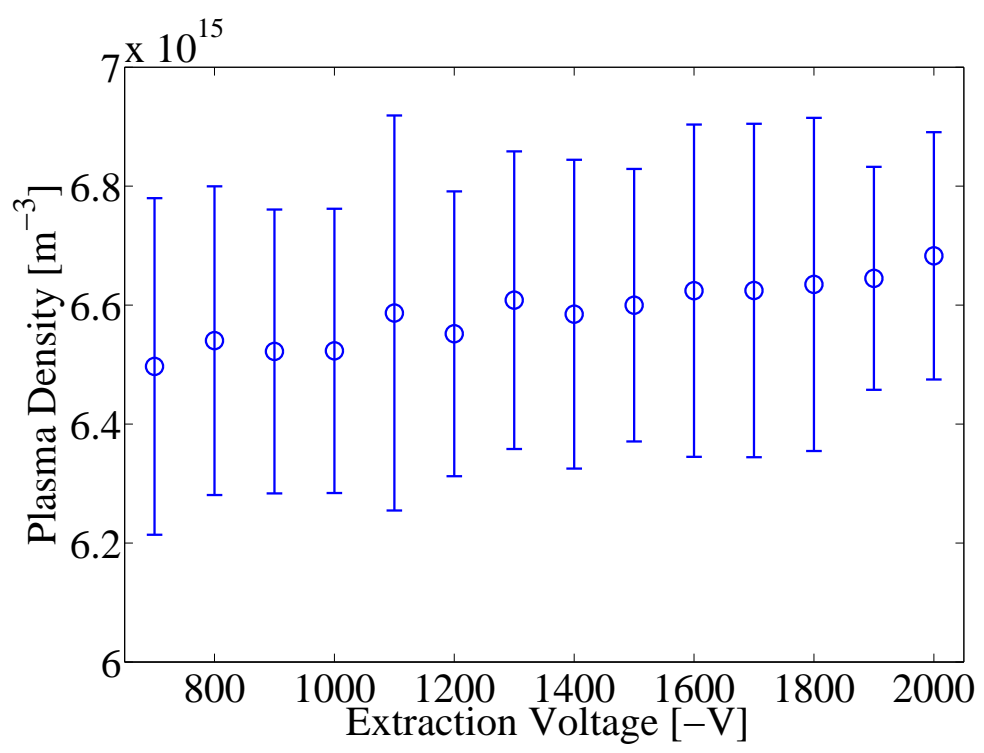

Figure 4.17: Estimated electron density of the plasma over a range of dc extraction voltages from 700 to $2000 \mathrm{~V}$ with a $0.3 \mathrm{~mm}$ hollow anode radius, an $\mathrm{RF}$ input voltage of $350 \mathrm{~V}$, an internal pressure of 1.25 Torr, and $\mathrm{H}_{3}{ }^{+}$ions. The density was corrected to account for collisions and scattering in the sheath surrounding the electrostatic probe.

Table 4.4: Modeled and average measured particle density for the extracted ion current over a range of dc extraction voltages from -700 to $-2000 \mathrm{~V}$ with a $0.3 \mathrm{~mm}$ hollow anode radius and an internal pressure of 1.25 Torr assuming an electron temperature of $4.45 \mathrm{eV}$.

\begin{tabular}{ccccc}
\hline Assumed Ion & Modeled & Uncorrected Estimated & Corrected Estimated & Units \\
\hline \hline $\mathrm{H}^{+}$ & $1.2 \times 10^{15}$ & $3.2 \times 10^{16}$ & $3.4 \times 10^{15}$ & $\mathrm{~m}^{-3}$ \\
$\mathrm{H}_{2}{ }^{+}$ & $1.9 \times 10^{15}$ & $4.5 \times 10^{16}$ & $4.6 \times 10^{15}$ & $\mathrm{~m}^{-3}$ \\
$\mathrm{H}_{3}{ }^{+}$ & $2.5 \times 10^{15}$ & $5.6 \times 10^{16}$ & $6.6 \times 10^{15}$ & $\mathrm{~m}^{-3}$ \\
\hline
\end{tabular}




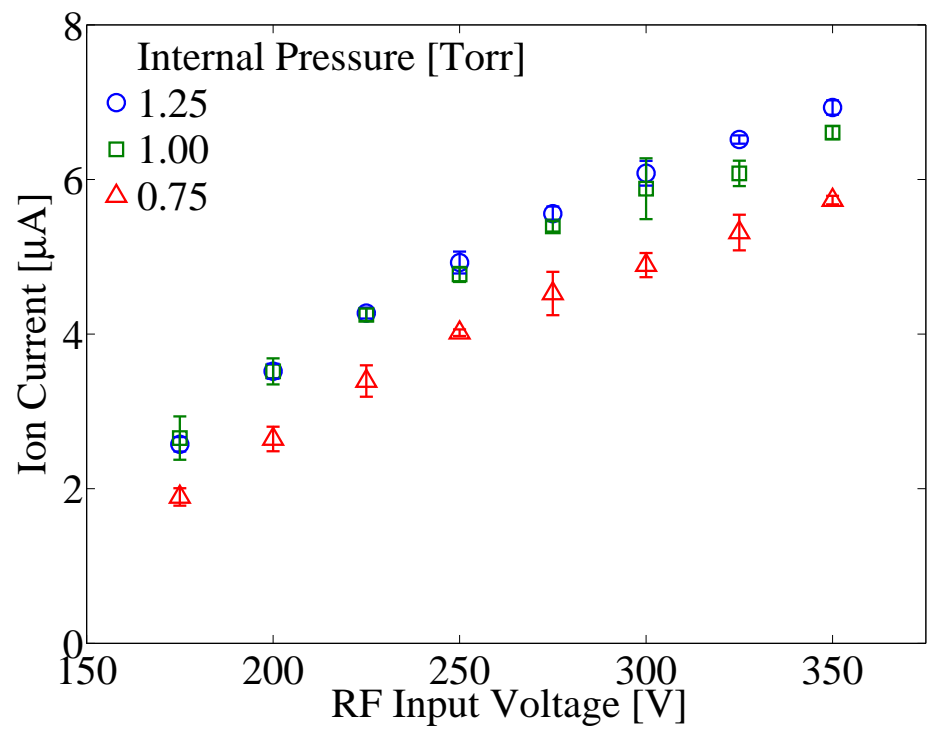

Figure 4.18: Measured hydrogen ion current for three internal pressures over a range of $\mathrm{RF}$ driving voltages with a $0.3 \mathrm{~mm}$ hollow anode radius and an extraction voltage of $-2000 \mathrm{~V}$.

Fig. 4.19 shows the electron temperature as it relates to the RF input voltage and internal pressure. Electron temperatures ranged from 1.75 to $4.44 \mathrm{eV}$, with the highest electron temperatures measured at the highest input voltage and internal pressure. The electron temperature showed an increase with increasing RF input voltage and internal pressure.

The electron density was calculated and corrected as described in Section 3.4 for a plasma consisting of $\mathrm{H}_{3}{ }^{+}$ions. As shown in Fig. 4.20, the electron densities ranged from $1.7 \times 10^{15} \mathrm{~m}^{-3}$ to $7.9 \times 10^{15} \mathrm{~m}^{-3}$, with the highest values measured at the highest RF input voltage and internal pressure.

\subsection{Ion Current Model}

Based on the results of ion source characterization, the ion current model described in Section 2.1 was used to determine the influence of electron 


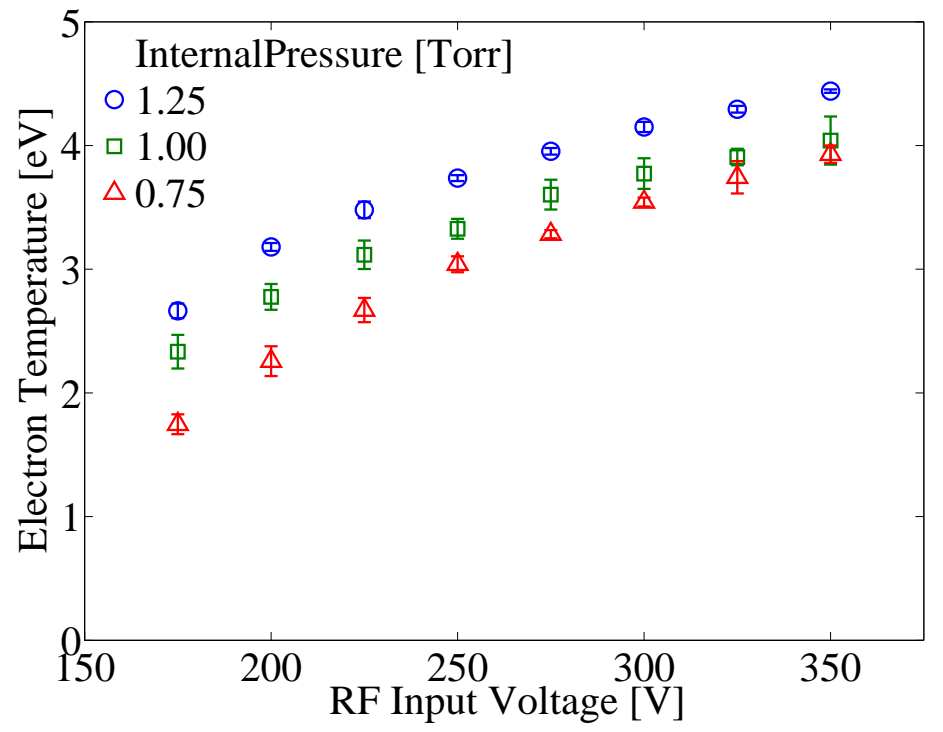

Figure 4.19: Measured electron temperature for three internal pressures over a range of RF driving voltages with a $0.3 \mathrm{~mm}$ hollow anode radius and an extraction voltage of $-2000 \mathrm{~V}$.

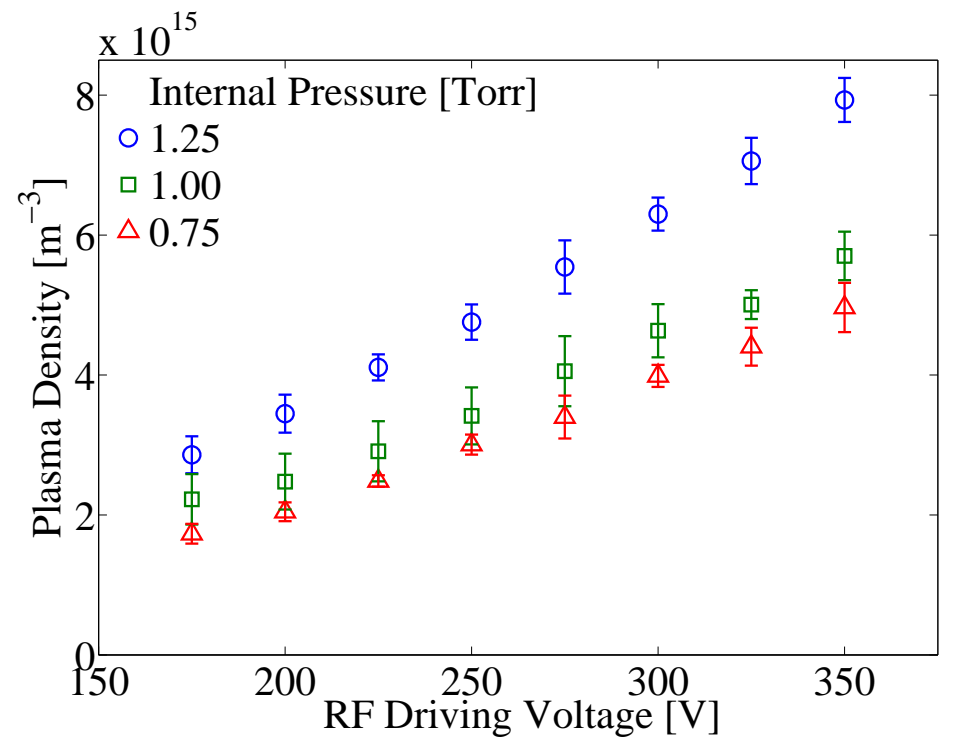

Figure 4.20: Measured particle density corrected for $\mathrm{H}_{3}{ }^{+}$ions for three internal pressures over a range of $\mathrm{RF}$ driving voltages with a $0.3 \mathrm{~mm}$ hollow anode radius and an extraction voltage of $-2000 \mathrm{~V}$. 
temperature and density on the ion current extracted from the plasma and was used to approximate the electron current and density of some of the extracted ion currents.

To determine degree of impact of electron density and temperature on the ion current produced by the PTPS, a large and small sweep of electron densities and a small sweep of the electron temperature were performed within the acceptable operating range for a glow discharge plasma. In Figs. 4.21, 4.22, and 4.23 the average measured hydrogen ion current for two data collections with a $400 \mathrm{~V}$ amplitude $\mathrm{RF}$ driving voltage, $0.3 \mathrm{~mm}$ radius hollow anode, $\mathrm{H}_{3}{ }^{+}$ion, and 2.5 Torr internal pressure was included for reference. In Figs. 4.21 and 4.22 the electron temperature is held at a constant $7 \mathrm{eV}$ while the electron density is changed. In Fig. 4.21, the densities are seperated by an order of magnitude to show the sensitivity of the ion current model to the order of magnitude of the electron density. Fig. 4.22 shows the impact of changing the electron density by $1 \mathrm{~m}^{-3}$. The electron temperature sweep, shown in Fig. 4.23, indicates that the ion current is affected by the change in electron temperature similarly to the electron density at small increments. Based on these calculations, the order of magnitude of the electron density has the greatest affect on the ion current.

Fig. 4.24 shows the measured hydrogen ion current for two data collections with a $400 \mathrm{~V}$ amplitude $\mathrm{RF}$ driving voltage, $0.3 \mathrm{~mm}$ radius hollow anode, $\mathrm{H}_{3}{ }^{+}$ ion, and 2.5 Torr internal pressure. Also shown in Fig. 4.24 is the modeled current assuming an electron density of $7.16 \times 10^{15} \mathrm{~m}^{-3}$ and electron temperature $4.44 \mathrm{eV}$. Based on the non-linear least squares fit of the listed parameters the electron density was found to be on the order of $10^{16} \mathrm{~m}^{-3}$ at 2.5 Torr. Table 4.5 shows the results of the non-linear least squares fit for electron temperatures from 1 to $10 \mathrm{eV}$, further demonstrating the minimal change in the electron density with the changing temperature. 


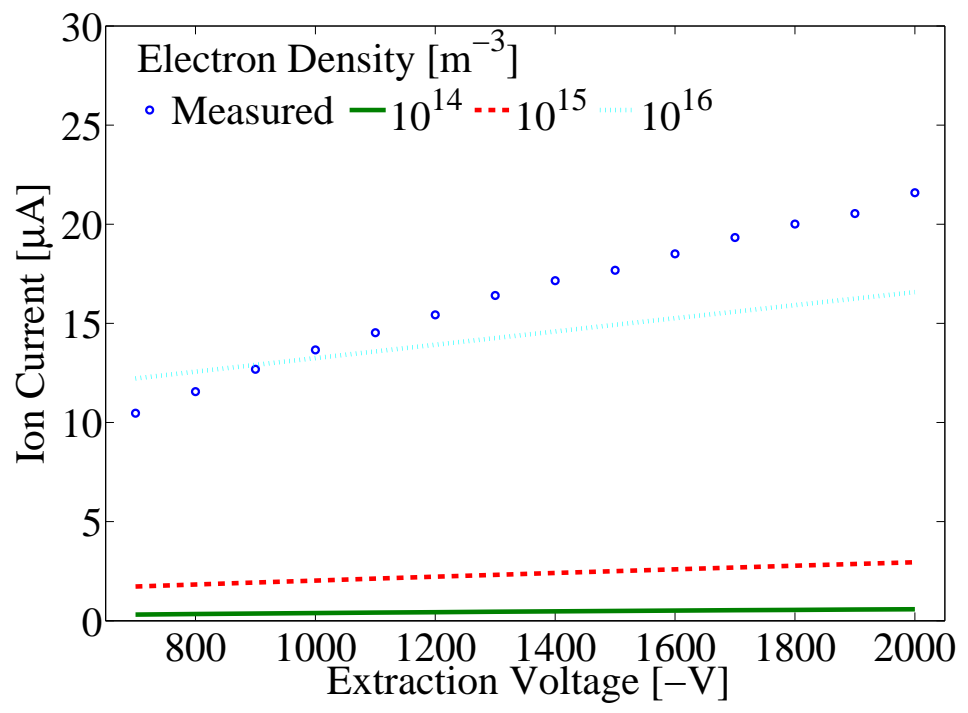

Figure 4.21: Measured and calculated ion current. The calculated current shown here used three densities and a fixed electron temperature of $7 \mathrm{eV}$. Measured current found hydrogen with a $0.3 \mathrm{~mm}$ hollow anode radius, $400 \mathrm{~V}$ RF driving voltage, and 2.5 Torr internal pressure over a range of dc extraction voltages from 200 to $2000 \mathrm{~V}$.

Table 4.5: Electron density found using the non-linear least square fit of the ion current model for electron temperatures from 1 to $10 \mathrm{eV}$ for the hydrogen ion current shown in Fig. 4.24.

\begin{tabular}{cc}
\hline Electron Temperature $[\mathrm{eV}]$ & Electron Density $\left[\times 10^{16} \mathrm{~m}^{-3}\right]$ \\
\hline \hline 1 & 2.5 \\
2 & 1.9 \\
3 & 1.6 \\
4 & 1.5 \\
5 & 1.3 \\
6 & 1.2 \\
7 & 1.2 \\
8 & 1.1 \\
9 & 1.0 \\
10 & 1.0 \\
\hline
\end{tabular}




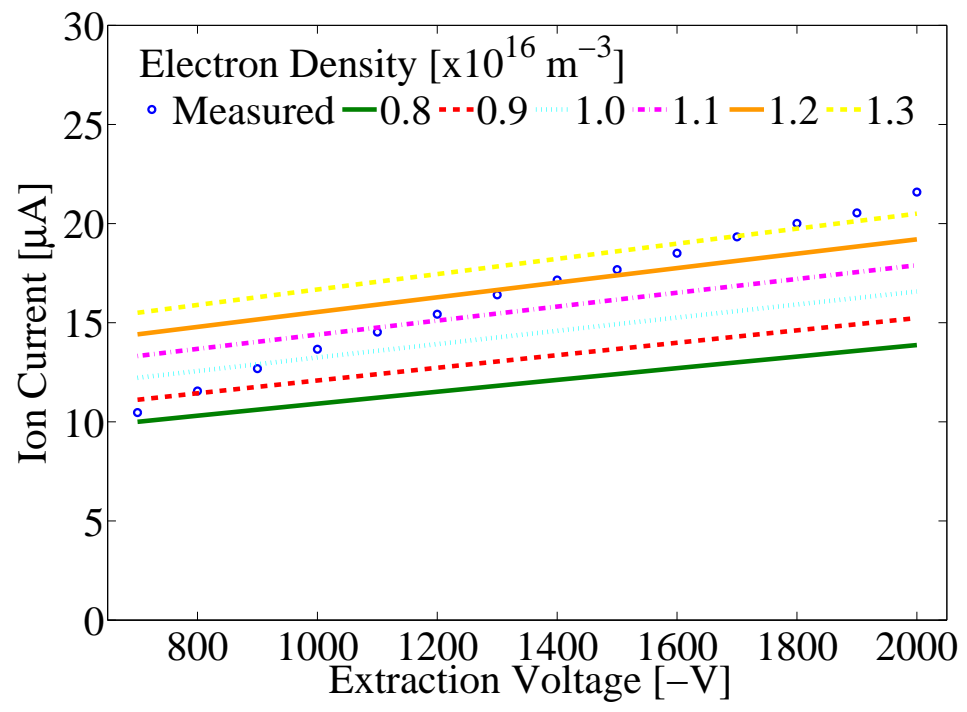

Figure 4.22: Measured and calculated ion current. The calculated current shown here used densities in the $10^{16} \mathrm{~m}^{-3}$ range and a fixed electron temperature of $7 \mathrm{eV}$. Measured current found hydrogen with a $0.3 \mathrm{~mm}$ hollow anode radius, $400 \mathrm{~V}$ RF driving voltage, and 2.5 Torr internal pressure over a range of dc extraction voltages from 200 to $2000 \mathrm{~V}$. 


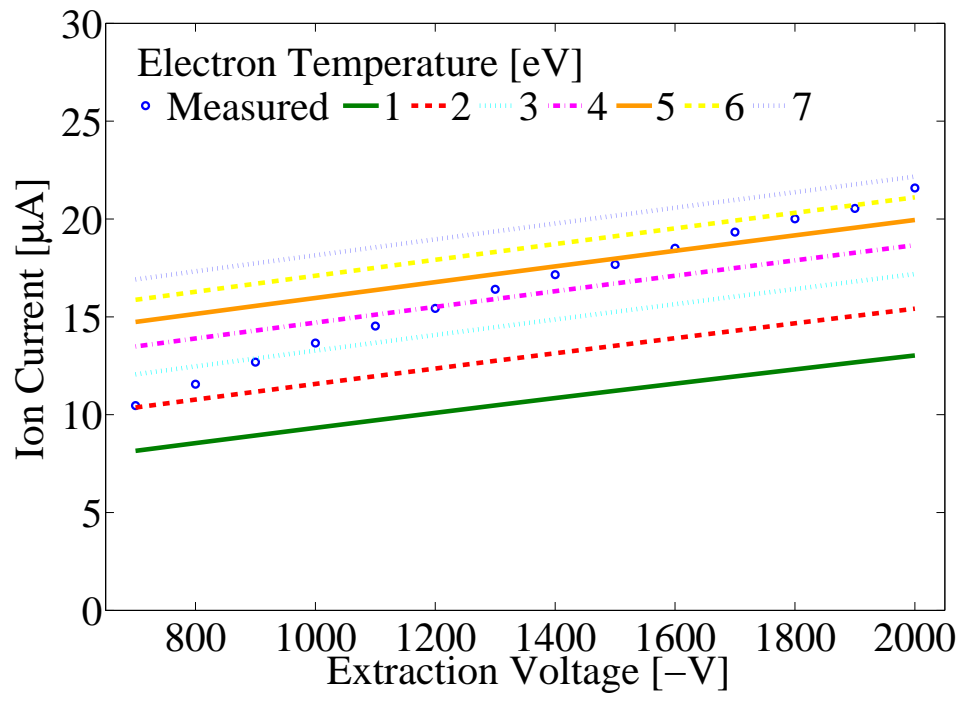

Figure 4.23: Measured and calculated ion current. The calculated current shown here used a fixed density of $1.4 \times 10^{16} \mathrm{~m}^{-3}$ and a range of temperatures from 1 to $7 \mathrm{eV}$. Measured current found hydrogen with a $0.3 \mathrm{~mm}$ hollow anode radius, $400 \mathrm{~V}$ RF driving voltage, and 2.5 Torr internal pressure over a range of dc extraction voltages from 200 to $2000 \mathrm{~V}$. 


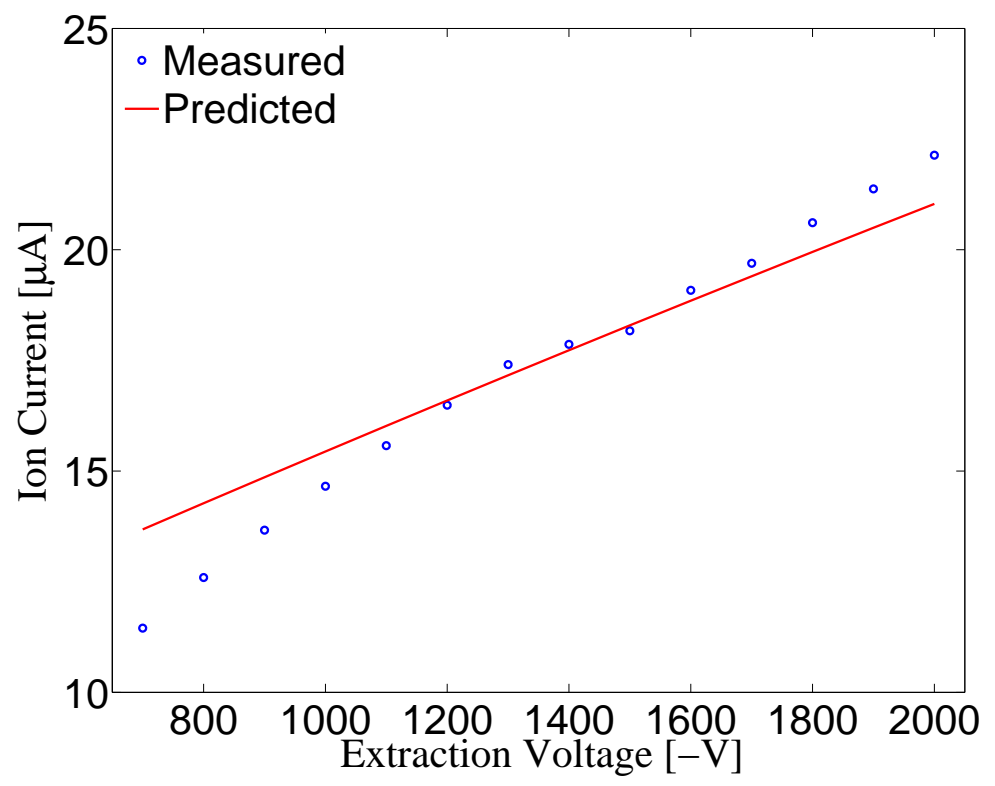

Figure 4.24: Measured and predicted hydrogen current for a $400 \mathrm{~V}$ input voltage, 2.5 Torr internal pressure, $0.3 \mathrm{~mm}$ radius hollow anode, $4.44 \mathrm{eV}$ electron temperature and $7.16 \times 10^{15} \mathrm{~m}^{-3}$ electron density. 


\section{Chapter 5}

\section{Conclusions}

The piezoelectric transformer plasma source (PTPS) was characterized as an ion source for neutron production. The PTPS was tested to determine the impact of several input parameters to the system on ion current to determine the optimal operating conditions of the source. The PTPS was also integrated into a neutron generation system to confirm that the PTPS was capable of producing the ion current outputs required for neutron production. The electrostatic probe method was used to determine the influence of PTPS input parameters on the electron temperature and density of the plasma. Finally, the PTPS results were compared with the ion current model to show comparisons between the model predictions and results. The ion current model was also used to predict the electron temperature and density given the measured ion current and input parameters of the PTPS.

\subsection{Ion Source Characterization Experiment}

The PTPS was characterized as a source of ions for neutron production to determine if the ion source was scalable and comparable with the ion current model presented in Section 2.1. Ion currents were measured using an extraction system with varied ionizing gases, extraction voltages, extraction gap lengths, 
RF driving voltages, hollow anode sizes, internal gas pressures, chamber depths, and operating durations. The ion current was assumed to be a function of the extraction electric field of the measurement system and the ion mass, electron temperature, and electron density of the plasma.

The PTPS was tested using hydrogen and deuterium gases to determine a functional relationship between the two. The ion currents showed a functional relationship of $I_{D} \sim I_{H} / \sqrt{2}$, where $I_{D}$ is the deuterium ion current and $I_{H}$ is the hydrogen ion current. This relationship is consistent with the Bohm criterion. Once a functional relationship was established between the hydrogen and deuterium gases, further testing was performed with hydrogen gas.

The impact of the extraction electric field on the ion current was tested by changing the extraction voltage, and the gap length between the hollow anode and secondary electron suppression grid. The extraction voltage was swept from -100 to $-2000 \mathrm{~V}$ for three different RF driving voltages and plotted in perveance to show that the ion current is in the space-charge limited region. The measured ion current increased with the increasing extraction voltages and RF input voltages. To further test the affects of the extraction electric field, the PTPS was tested with extraction gap lengths ranging from 10 to $25 \mathrm{~mm}$ for two internal pressures and the same range extraction voltages. The current reached the space-charge limit for each of the internal pressures and gaps. At the larger gap lengths, the extraction electric field applied was limited by the maximum amount of extraction voltage that could be supplied to the system, but at each gap length the space-charge limit is reached at $-300 \mathrm{~V}$ extraction. The results also showed that the ion current measured had a similar trend at both internal pressures, with the higher currents measured at the higher pressure.

The electron density and temperature of the plasma were assumed to be functions of the RF driving voltage, hollow anode radius, internal pressure, and ionizing chamber depth. The electric field ionizing the supplied gas was a function 
of the RF driving voltage and the ionizing chamber depth. To determine the impact of RF driving voltage on ion current, the RF driving voltage was varied from 200 to $400 \mathrm{~V}$. At the maximum extraction voltage, doubling the RF driving voltage (from 200 to $400 \mathrm{~V}$ ) resulted in more than triple the ion current. To determine the influence of chamber depth on the ion current, chamber depths of 3,6 , and $9 \mathrm{~mm}$ were used resulting in the highest ion currents measured at the smallest depth. The internal pressure of the ionizing chamber was controlled by the flow rate of gas into the PTPS and the hollow anode through which ions were extracted. Doubling the hollow anode size resulted in a significant ion current increase, while increasing the flow rate to double the internal pressure resulted in an ion current increase of 1.6 times. Additional internal pressure testing showed that, within the capabilities of the gas flow equipment, the ion current did not reach a maximum value for the internal pressure.

To function as an ion source for neutron production, the PTPS would need to be able to operate for extended periods of time. Tests confirmed that the PTPS was able to function for lengths of time ranging from 4 to 10 hours. The initial measured currents were approximately $50 \%$ higher than ion currents measured 45 minutes after the PTPS operation was started. Based on these results the PTPS was operated for at least 45 minutes before experiments were performed.

The PTPS ion current was characterized to determine the optimal operating parameters. The ionizing gas, extraction voltage, extraction gap length, RF driving voltage, ionization chamber depth, hollow anode size, internal gas pressure, and operation duration were tested. Based on the results of these experiments, the PTPS is a scalable ion source capable of producing ion currents in the micro-Ampere range, with the maximum measured ion current of $27 \mu \mathrm{A}$ for hydrogen gas, which corresponds to $19 \mu \mathrm{A}$ for deuterium gas. 


\subsection{Neutron Generation Experiment}

The PTPS was integrated into a system for neutron production with a dc high voltage source supplying the ion accelerating voltage to a deuterium-doped titanium target. The neutron counts generated by the system were measured for various dc accelerating voltages, RF driving voltage, internal pressure, and operating pulse repetition rate. Neutron counts above background were measured measured at each accelerating voltage, with no false alarm probability (FAP) at accelerating voltages greater than $92 \mathrm{kV}$. No discernible trend was observed with the change of the RF driving voltage, internal pressure, and operating pulse repetition rate. Based on the neutron generation experiment, the PTPS is a viable ion source for neutron production, especially at accelerating voltages greater than $92 \mathrm{kV}$.

\subsection{Electrostatic Probe Experiment}

The electron density and temperature of the plasma were estimated using the triple electrostatic probe method to determine the influence of extraction voltage, RF driving voltage, and internal pressure of the PTPS on the fundamental parameters of the plasma. The extraction voltage testing showed that while ion current increased as the extraction voltage increased, the electron temperature and density remained constant. The internal pressure and RF input voltage testing showed an increase in ion current, electron temperature, and electron density with the increase in internal pressure and RF driving voltage. Based on these measurements the PTPS operated between 0.75 and 1.25 Torr produced electron temperatures in the range of 1.75 to $4.44 \mathrm{eV}$ and electron densities in the range of $1.7 \times 10^{15}$ to $7.9 \times 10^{15} \mathrm{~m}^{-3}$, which are within the acceptable range for a glow discharge plasma. 


\subsection{Ion Current Model}

Comparisons of the ion current model, described in Section 2.1, with the results of the ion source characterization experiment and electrostatic probe experiment showed that the ion current model could be used to predict the output of the PTPS, though the hollow anode radius had a larger impact on experimental results than the model had predicted. Calculations were performed to show that the order of magnitude of the electron density of the plasma had a significant influence on the ion current, while the electron temperature had much less of an impact.

\subsection{Summary}

The PTPS was tested as an ion source for neutron production. It was characterized to determine that it was a scalable ion source capable of ion currents in the miro-Ampere range. The PTPS was integrated into a neutron generator system, proving that the ion currents produced were sufficient for neutron counts above background. The electron density and temperature were measured using an electrostatic probe system, and agreed with the order of magnitude approximation of the ion current model and the accepted values for a glow discharge plasma. Finally, the ion current model was shown to agree with the results of the PTPS testing, allowing for approximations of the ion current from the PTPS. Based on these experiments, the PTPS could be used as an ion source for neutron production, but would require increased ion currents to make it competitive with existing technologies. 


\section{Bibliography}

[1] S. Nargolwalla and E. Przyblowicz, Activation Analysis with Neutron Generators. John Wiley and Sons, 1973.

[2] "Neutron generators for analytical purposes," International Atomic Energy Agency, Vienna, Tech. Rep. IAEA Radiation Technology Reports Series No. 1, 2012.

[3] A. Lorenz, "Survey of neutron sources and their applications," Lawerence Livermore Laboratory, Tech. Rep. UCRL-51298, January 1972.

[4] J. Walker, "Uses of neutrons in engineering and technology," Physics and Technology, vol. 13, pp. 239-248, November 1982.

[5] R. Runkle, T. White, E. Miller, J. Caggiano, and B. Collins, "Photon and neutron interrogation techniques for chemical explosives detection in air cargo: a critical review," Nuclear Instruments and Methods in Physics Research A, vol. 603, pp. 510-528, 2009.

[6] R. Hertzog and R. Plasek, "Neutron-excited gamma ray spectrometry for oil well logging," IEEE Transactions on Nuclear Science, vol. 26, no. 1, February 1979.

[7] C. Moss, C. Goulding, C. Hollas, and W. Myers, "Neutron detectors for active interrogation of highly enriched uranium," IEEE Transactions on Nuclear Science, vol. 51, no. 4, pp. 1677-1681, August 2004.

[8] W. Myers, "Photon and neutron active interrogation of highly enriched uranium," in Proceedings of American Institute of Physics, vol. 769, 2005.

[9] D. Chichester, J. Simpson, and M. Lemchak, "Advanced compact accelerator neutron generator technology for active neutron interrogation field work," Jounal of Radioanalytical and Nuclear Chemistry, vol. 271, pp. 629-637, 2007. 
[10] A. Benwell, S. Kovaleski, and M. Kemp, "A high-voltage piezoelectric transformer for active interrogation," Journal of Nuclear Materials Management, vol. 38, pp. 42-47, 2008.

[11] B. Ludewigt, R. Wells, and J. Reijonen, "High-yield D-T neutron generator," Nuclear Instruments and Methods in Physics Research B, vol. 261, pp. 830-834, 2007.

[12] V. Lacoste, "Review of radiation sources, calibration facilities and simulated workplace fields," Radiation Measurements, vol. 45, pp. 10831089, 2010.

[13] J. Reijonen, F. Gicquel, S. Hahto, M. King, T.-P. Lou, and K.-N. Leung, "D-D neutron generator development at LBNL," Applied Radiation Isotopes, vol. 63, pp. 757-763, 2005.

[14] D. Price and J. Rush, "Neutron sources and applications," Department of Energy, Tech. Rep. DOE/ER-0607P, January 1994.

[15] A. Smith and P. Fields, "Spontaneous fission neutron spectrum of cf-252," Physical Review, vol. 195, pp. 403-409, 1957.

[16] R. Smith, C. Bush, and J. Reichart, "Small accelerators as neutron generators for the borehole environment," IEEE Transactions on Nuclear Science, vol. 35, no. 1, pp. 859-862, February 1988.

[17] O. Serra, Fundamentals of well-log interpretation 1. the acquisition of logging. Elsevier, 1984.

[18] S. Kerr and P. Worthington, "Nuclear logging techniques for hydrocarbon, mineral and geological applications," IEEE Transactions on Nuclear Science, vol. 35, pp. 794-799, 1988.

[19] A. Chen, A. Antolak, and K.-N. Leung, "Electronic neutron sources for compensated porosity well logging," Nuclear Instruments and Methods in Physics Research A, vol. 684, pp. 52-56, 2012.

[20] W. Gilchrist, F. Inanc, and L. Roberts, "Nuclear source replacement promises and pitfalls," in SPWLA 52nd Annual Logging Symposium, 2011.

[21] M. Skidmore, R. Ambrosi, and R. O’Brien, "Neutron sourcces for in situ planetary science applications," Nuclear Instruments and Methods in Physics Research A, vol. 608, pp. 403-409, 2009. 
[22] L. Xu, W. Schultz, and C. Huiszoon, "A comprehensive investigation of source effects on neutron porosity response for logging-while-drilling measurements," in SPWLA 50th Annual Logging Symposium, The Woodlands, Texas, June 21-24 2009.

[23] D. Chichester and E. Seabury, "Using electronic neutron generators in active interrogation to detect shielded fissionable material," in Nuclear Science Symposeum Conference, Dresden, Germany, 2008, pp. 3361-3367.

[24] J. Elizondo-Decanini, D. Shchmale, M. Cich, M. Martinez, K. Youngman, M. Senkow, S. Kiff, J. Steele, R. Goeke, B. Wroblewski, J. Desko, and A. Dragt, "Novel surface-mounted neutron generator," IEEE Transactions on Plasma Science, vol. 40, pp. 2145-2150, 2012.

[25] H. Conrads and M. Schmidt, "Plasma generation and plasma sources," Plasma Sources Science and Technology, vol. 9, pp. 441-454, 1000.

[26] H. Loeb, "Plasma based ion beams," Plasma Physics and Controlled Fusion, vol. 47, pp. B565-B576, 2005.

[27] S. Nikiforov, V. Golubev, D. Solnyshkov, and M. Svinin, "Ion sources for use in research and applied high voltage accelerators," in Proceedings of the 1995 Particle Accelerators Conference, vol. 2, Dallas, TX, USA, 1995, pp. 1004-1006.

[28] I. Brown, Ed., The Physics and Technology of Ion Sources, 2nd ed. WileyVch, 2004.

[29] B. Wolf, Ed., Handbook of Ion Sources. CRC Press, 1995.

[30] F. Chen and J. Chang, Lecture Notes on Principles of Plasma Processing. Plenum/Kluwer Publishing, 2002.

[31] F. F. Chen, Introduction to Plasma Physics and Controlled Fusion Plasma Physics. Springer, 1995.

[32] B. Das and A. Shyam, "Development of compact size Penning ion source for compact neutron generator," Review of Scientific Instruments, vol. 79, p. $123305,2008$.

[33] W. Liu, M. Li, K. Gao, and D. Gu, "Discharge characteristics of a Penning ion source for compact neutron generator," Nuclear Instruments and Methods in Physics Research Section A, vol. 768, pp. 120-123, 2014. 
[34] G. Morris, C. Bush, and J. Reichart, "Small accelerators for borehole application," IEEE Transactions on Nuclear Science, vol. NS-30, pp. 16481651, 1983.

[35] J. Rovey, B. Ruzic, and T. Houlahan, "Simple Penningion source for laboratory research and development applications," Review of Scientific Instruments, vol. 78, p. 106101, 2007.

[36] R. Smith, C. Bush, and J. Reichart, "Small accelerators as neutron generators for the borehole environment," IEEE Transactions on Nuclear Science, vol. 35, pp. 859-862, 1988.

[37] A. Sy, Q. Ji, A. Persaud, O. Waldmann, and T. Schenkel, "Novel methods for improvements of a Penning ion source for neutron generator applications," Review of Scientific Instruments, vol. 83, p. 02B309, 2012.

[38] H. Jung, M. Park, S. Kim, and Y. Hwang, "Development of a compact helicon ion source for neutron generators," Review of Scientific Instruments, vol. 75, pp. 1878-1880, 2004.

[39] I. Kim, N. Jung, H. Jung, Y. Hwang, and H. Choi, "A D-D neutron generator using a titanium drive-in target," Nuclear Instruments and Methods in Physics Research Section B, vol. 266, pp. 829-833, 2008.

[40] J. Verbeke, K. Lenug, and J. Vujic, "Development of a sealed-acceleratortube neutron generator," Applied Radiation and Isotopes, vol. 53, pp. 801$809,2000$.

[41] Y. Wu, J. Hurley, Q. Ji, J. Kwan, and K. Leung, "Sealed operation of a RF driven ion source for a compact neutron generator to be used for associated particle imaging," Review of Scientific Instruments, vol. 81, p. 02B908, 2010.

[42] J. Reijonen, "Neutron generators developed at LBNL for homeland security and imaging applications," Nuclear Instruments and Methods in Physics Research B, vol. 261, pp. 272-276, 2007.

[43] W. Wang, Y. Song, J. Wang, Y. Wang, and G. Song, "Design of the 2.45 $\mathrm{GHz}$ ECR ion source control system for high intensity D-T fusion neutron generator," Journal of Fusion Energy, vol. 34, pp. 584-591, 2015. 
[44] B. Bogdanovich, A. Didenko, A. Nesterovich, A. Prokopenko, V. Shatokhin, and A. Shikanov, "Nuetron generators based on microwave sources of hyeavy hydrogen nuclides," Instruments and Experimental Techniques, vol. 58, pp. 447-455, 2015.

[45] S. Jing, W. Li, L. Gu, and L. Liu, "The ceramic-cup microwave ion source for sealed-tube neutron generator," Review of Scientific Instruments, vol. 72, pp. 92-95, 2001.

[46] V. S. Kalyga, I. Izotov, S. Golubev, S. Razin, A. Sidorov, A. Maslennikova, A. Volovecky, T. Kalvas, H. Koivisto, and O. Tarvainen, "Gyrotron-driven high current ECR ion source for boron-neutron capture therapy neutron generator," Nuclear Instruments and Methods in Physics Research Section A, vol. 768, pp. 146-150, 2014.

[47] J. Vainionpaa, J. Harris, M. Piestrup, C. Gary, D. Williams, M. Apodaca, J. Cremer, Q. Ji, B. Ludewigt, and G. Jones, "High yield neutron generators using the DD reaction," in AIP Conference Proceedings, Fort Worth, TX, USA, 2013, pp. 118-122.

[48] H. Itoh, K. Teranishi, and S. Suzuki, "Discharge plasmas generated by piezoelectric transformers and their applications," Plasma Sources Science and Technology, vol. 15, pp. S51-S61, 2006.

[49] J. Curie and P. Curie, "Development by pressure of polar electricity in hemihedral crystals with inclined faces," Bulletin of the Society of French Mineralists, vol. 3, pp. 90-93.

[50] N. Setter, Piezoelectric Materials in Devices, N. Setter, Ed. Lausanne: Ceramics Laboratory, EPFL, 2002.

[51] C. Rosen, in Process Electronics COmponent Symposium, 1957, p. 205.

[52] K. Teranishi, S. Suzuki, and H. Itoh, "A neovel generation method of dielectric barrier discharge and ozone production using a piezoelectric transformer," Japanese Journal of Applied Physics, vol. 43, no. 9B, pp. 6733-6739, 2004.

[53] B. Hutsel, "Characterization of a piezoelectric transformer plasma source," Ph.D. dissertation, University of Missouri, 2012.

[54] A. Benwell, "A high voltage piezoelectric transformer for active interrogation," Ph.D. dissertation, University of Missouri, 2009. 
[55] M. Kemp, "The ferroelectric plasma thruster," Ph.D. dissertation, Department of Electrical and Computer Engineering, University of Missouri, Columbia, MO, 2008.

[56] K. Teranishi, S. Suzuki, and H. Itoh, "Luminous phenomenon of silent discharge using a piezoelectric transformer," Japanese Journal of Applied Physics, vol. 40, pp. 5766-5768, 2001.

[57] K. Ternashi, S. Suzuki, and H. Itoh, "Dynamic behavior of litght emissions generated by piezoelectric transformers," IEEE Transactions on Plasma Science, vol. 30, no. 1, pp. 122-123, February 2002.

[58] H. Itoh, K. Teranishi, and S. Suzuki, "Observation of light emissions around a piezoelectric transformer in various gases," IEEE Transactions on Plasma Science, vol. 30, no. 1, pp. 124-125, February 2002.

[59] K. Teranishi, S. Suzuki, and H. Itoh, "Luminescence from fluorescent material excited by piezoelectric transformer," Japanese Journal of Applied Physics, vol. 41, no. 11B, pp. 7160-7165, November 2002.

[60] K. Ternashi, S. Suzuki, and H. Itoh, "Dielectric barrier dishcharge generated by pizoelectric transformer in atmospheric pressure," IEEE Transactions on Plasma Science, vol. 33, no. 2, pp. 296-297, April 2005.

[61] H. Itoh, K. Teranishi, and S. Suzuki, "Glow discharge around piezoelectric transformer operate under higher-order vibration modes," IEEE Transactions on Plasma Science, vol. 33, no. 2, pp. 352-353, April 2005.

[62] K. Teranishi and H. Itoh, "Absolute measurement of surface potential and discharge power distributions for piezoelectric transformer-based plasma reactor," Japanese Journal of Applied Physics, vol. 44, no. 9B, pp. 7083$7088,2005$.

[63] H. Itoh, T. Suzuki, S. Suzuk, and I. Rusinov, "Investigation of ozone loss rate influenced by the surface material of a discharge chamber," Ozone Science and Engineering, vol. 24, pp. 487-497, 2004.

[64] H. Itoh, I. Ruisinov, S. Suzuki, and T. Suzuki, "Variation of ozone reflection coefficient at a metal surface with its gradual oxidation," Plasma Processing of Polymers, vol. 2, no. 3, pp. 227-231, March 2005. 
[65] A. Flynn and S. Sanders, "Fundametnal limits on energy transfer and circuit considerations for piezoelectric transformers," IEEE Transactions on Power Electronics, vol. 17, pp. 8-14, January 1992.

[66] R. Weis and T. Gaylord, "Lithium niobate: summary of physical properties and crystal structure," Applied Physics A, vol. 37, pp. 191-203, 1985.

[67] I. Brown, "Vacuum arc ion sources," Review of Scientific Instruments, vol. 65, no. 10, pp. 3061-3081, October 1994.

[68] R. Keller, D. Cheng, R. DiGennaro, R. Gough, J. Greer, K. Leung, A. Ratti, J. Reijonen, R. Thomae, T. Schenkel, J. Staples, R. Yourd, A. Aleksandrov, M. Stockli, and R. Welton, "Ion-source and low-energy beam-transport issues with the front-end systems for the spallation neutron source," Review of Scientific Instruments, vol. 73, no. 2, pp. 914-916, February 2002.

[69] S. Lawrie, D. Faircloth, A. Letchford, C. Gabor, and J. Pozimski, "Plasma meniscus and extraction electrode studies of the ISIS H- ion source," Review of Scientific Instruments, vol. 81, p. 02A707, 2010.

[70] M. Menzinger and L. Wahlin, "High intensity, low energy spread ion source for chemical accelerators," Review of Scientific Instruments, vol. 40, no. 1, pp. 102-105, January 1969.

[71] R. Davis, O. Morgan, L. Stewart, and W. Stirling, "A multiampere duoPIGatron ion source," Review of Scientific Instruments, vol. 43, no. 2, pp. 278-283, February 1972.

[72] M. Turek, A. Drodziel, K. Pyszniak, and J. Sielanko, "Extraction of the ion beam from hollow cathode ion source. experiment and computer simulation," in Proccedings of the Fifth Internaional Conference on Ion Implantation and other Applications of Ions and Electrons, vol. 78, 2004, pp. $649-654$.

[73] S. Kovaleski, "Calculation of the ion extraction boundary of a plasma ion source," IEEE Transactions on Plasma Science, vol. 34, no. 1, pp. 23-27, February 2006.

[74] J. Ke and C. Zhou, "A method for estimating the sheath edge of plasma ion sources," IEEE Transactions on Plasma Science, vol. 42, no. 4, pp. 944-947, April 2014. 
[75] M. Hassouba, A. Galay, and U. Rashed, "Analysis of cylindrical Langmuir probe using experiment and different theories," Plasma Physics Reports, vol. 39, no. 3, pp. 255-262, 2013.

[76] R. Kiel, "Continuum electrostatic probe theory for large sheaths on spheres and cylinders," Journal of Applied Physics, vol. 40, no. 9, pp. 3668-3673, August 1969.

[77] J. Allen, "Probe theory - the orbital motion approach," Physica Scripta, vol. 45, pp. 497-503, 1992.

[78] B. Annaratone, M. Allen, and J. Allen, "Ion currents to cylindrical Langmuir probes in RF plasmas," Journal of Physics D: Applied Physics, vol. 25, pp. 417-424, 1992.

[79] C. Brandt, H. Testrich, R. Kozakov, and C. Wilke, "Investigation of the disturbance of a Langmuir probe and its influence on measurement results," Review of Scientific Instruments, vol. 77, p. 023504, 2006.

[80] J.-S. Chang and J. Laframboise, "Probe theory for arbitrary shape in a large Debye length, shtationary plasma," Physics of Fluids, vol. 19, pp. $25-31,1976$.

[81] F. Chen and D. Arnush, "The floating potential of cylindrical Langmuir probes," Physics of Plasmas, vol. 8, no. 11, pp. 5051-5052, November 2001.

[82] S.-L. Chen, J.-S. Chang, and S. Matsumura, "Electrostatic probe determination of electron density in medium pressure (0.2-28 Torr) discharge plasma of argon," Journal of Applied Physics, vol. 41, pp. 1711$1715,1970$.

[83] F. Chen, "Langmuir probes in RF plasma: surprising validity of OML theory," Plasma Sources Science and Technology, vol. 18, p. 035012, 2009.

[84] S.-L. Chen and T. Sekiguchi, "Instantaneous direct-display system of plasma parameters by means of triple probe," Journal of Applied Physics, vol. 36 , no. 8, pp. 2363-2375, August 1965.

[85] B. Cherrington, "The use of electrostatic probes for plasma diagnostics a review," Plasma Chemistry and Plasma Processing, vol. 2, no. 2, pp. 113-139, 1982. 
[86] P. Chung, L. Talbot, and K. Touryan, "Electric probes in stationary and flowing plasmas: part 1. collisionless and transitional probes," AIAA Journal, vol. 12, no. 2, pp. 133-144, February 1974.

[87] F. Crawford, "Modulated Langmuir probe characteristics," Journal of Applied Physics, vol. 34, no. 7, pp. 1897-1902, July 1963.

[88] M. Kamitsuma and S. Teii, "Theory of a spheroidal probe in low-density continuum plasmas," Pysics of Fluids, vol. 25, pp. 1169-1173, 1982.

[89] C. Deline, E. Choiniere, and B. Gilchrist, "Assessment of plasma-flow effect on Langmuir triple-probe operation via Kinetic simulation," IEEE Transactions on Plasma Science, vol. 37, no. 37, pp. 1843-1849, September 2009.

[90] V. Demidov, N. Kovolov, and A. Kurdryavstev, Probe Methods for LowTemperature Plasma Investigations. Energoatomizdat, 1996.

[91] V. Demidov, S. Ratynskaia, and K. Rypdal, "Electric probes for plasmas: The link between theory and instrument," Review of Scientific Instruments, vol. 73, pp. 3409-3439, 2002.

[92] S. Ghosh, K. Barada, P. Chattopadhyay, J. Ghosh, and D. Bora, "Resolving an anomaly in electron temperature measurement using double and triple Langmuir probe," Journal of Vacuum Technology, vol. 24, pp. $015017-$ 015 026, 2015.

[93] R. Estes and J. Sanmartin, "Cylindrical Langmuir probes beyond orbitalmotion-limited regime," Physics of Plasmas, vol. 7, no. 10, pp. 4320-4325, October 2000.

[94] V. Godyak, R. Piejak, and B. Alexandrovich, "Electron energy distribution function measurements and plasma parameters in inductively coupled argon plasma," Plasma Sources Science and Technology, vol. 11, no. 1, pp. 525-543, 2002.

[95] H. Habiger, M. Auweter-Kurtz, and H. Kurtz, "Electrostatic probes for the investigation of arc-driven electric populsion devices," in IEPC93-124. Seattle, WA: 23rd International Electric Propulsion Conference, Sept 13-16 1993, pp. 1137-1147. 
[96] M. Hopkins and W. Graham, "Langmuir probe technique for plasma parameter measurement in a medium density discharge," Review of Scientific Instruments, vol. 57, no. 9, pp. 2210-2217, September 1986.

[97] J. Hopwood, C. Guarnieri, S. Whitehair, and J. Cuomo, "Langmuir probe measurements of a radio frequency induction plasma," Journal of Vacuum Science and Technology A, vol. 11, no. 1, pp. 152-156, Jan/Feb 1993.

[98] F. Iza and J. Lee, "Particle-in-cell simulations of planar and cylindrical Langmuir probes: floatining potential and ion saturation current," Journal of Vacuum Science and Technology, vol. 24, pp. 1366-1372, 2006.

[99] J. Jauberteau and I. Jauberteau, "Plasma parameters deduced from cylindrical probe measurements: determination of the electron density at the ion saturation current," Plasma Sources of Science and Technology, vol. 17, p. 015019, 2008.

[100] S. Jeon, G. Eom, J. Kim, and W. Choe, "Multi-channel rf-compensated langmuir probe array driven by a single bias supply," Review of Scientific Instruments, vol. 73, no. 2, pp. 277-282, February 2002.

[101] M. Kamitsuma and S.-L. Chen, "Experimental studies of the large Debye length probe theory in a continuum plasma," Journal of Applied Physics, vol. 48, no. 12, pp. 5347-5348, December 1977.

[102] M. Kamitsuma, S.-L. Chen, and J.-S. Chang, "The theory of the instantaneous triple-probe method for direct display of plasma parameters in low-density collisionless plasmas," Journal of Physics D: Applied Physics, vol. 10, pp. 1065-1077, 1977.

[103] R. Kirchoff, E. Peterson, and L. Talbot, "An experimental study of the cylindrical Langmuir probe response in the transition regime," AIAA Journal, vol. 9, no. 9, pp. 1686-1694, September 1971.

[104] J. Laframboise, "Theory of spherical and cylindrical Langmuir probes in a collisionless, Maxwellian plasma at rest," Toronto University Sownsview Inst for Aerospace Studies, Tech. Rep. UTIAS Reb. No. 100, June 1966.

[105] R. Little and J. Waymouth, "Experimentally determined plasma perturbation by a probe," Physics of Fluids, vol. 9, no. 4, pp. 801-808, April 1966. 
[106] S. Matsumura, A. Berezin, and J.-S. Chang, "Digitalized triple-probe system for the direct display of electron temperature in a time-varying plasma," Review of Scientific Instruments, vol. 63, pp. 1783-1787, 1992.

[107] M. Meier, G. Hallock, H. Tsui, and R. Bengston, "The time-domain tripleprobe method," Review of Scientific Instruments, vol. 66, no. 1, pp. 437439, January 1995.

[108] L. Meng, A. Cloud, S. Jung, and D. Ruzic, "Study of plasma dynamics in a modulated pulsed power magnetron discharge using a time-resolved Langmuir probe," Journal of Vacuum Science and Technology A, vol. 29, p. 011024, 2011.

[109] M. Naz, A. Ghaffar, N. Rehman, S. Naseer, and M. Zakaullah, "Double and triple Langmuir probes measurements in inductively coupled nitrogen plasma," Electromagnetic Waves, vol. 114, pp. 113-128, 2011.

[110] M. Naz, S. Shukrullah, A. Ghaffar, and N. Rehman, "Development of simple designs of multitip probe diagnostic systems for RF plasma characterization," Scientific World Journal, vol. 2014, pp. 1-8, 2014.

[111] G. Ogram, J.-S. Chang, and R. Hobson, "The triple-probe method applied to the direct display of plasma parameters in supersonic flowing continuum plasma," Journal of Applied Physics, vol. 50, no. 2, pp. 726-730, February 1979 .

[112] L. Oksuz, F. Soberon, and A. Ellingboe, "Analysis of uncompensated Langmuir probe characteristics in radio-frequency discahrges revisited," Journal of Applied Physics, vol. 99, p. 013304, 2006.

[113] L. Oksuz and N. Hershkowitz, "Plasma, presheath, collisional sheath and collisionless sheath potential profiles in weakly ionized, weakly collisional plasma," Plasma Sources Science and Technology, vol. 14, pp. 201-208, 2005 .

[114] L. Overzet and M. Hopkins, "Comparison of electron-density measurements made using a Langmuir probe and microwwave interferometer in the Gaseous Electronics Conference reference reactor," Journal of Applied Physics, vol. 74, no. 7, pp. 4323-4330, October 1993.

[115] A. Paranjpe, J. McVittie, and S. Self, "A tuned langmuir probe for measurements in rf glow discharges," Journal of Applied Physics, vol. 67, pp. 6718-6727, 1990. 
[116] E. Passoth, P. Kudrna, C. Csambal, J. Benhnke, M. Tichy, and V. Helbig, "An experimental study of plasma density determination by a cylindrical Langmuir probe at different pressures and magnetic fields in a cylindrical magnetron discharge in heavy rare gases," Jounrnal of Physics D: Applied Physics, vol. 30, pp. 1763-1777, 1997.

[117] H. Peorteanu, S. Kuhn, and R. Gesche, "Electric probe insvestigations of microwave generated, atmospheric pressure, plasma jets," Journal of Applied Physics, vol. 108, p. 013301, 2010.

[118] W. McColl, C. Brooks, and M. Brake, "Double Langmuir probe diagnostic of a resonant cavity microwave discharge," Journal of Vacuum Science and Technology A, vol. 11, pp. 1152-1155, 1993.

[119] C. Riccardi, G. Longoni, G. Chiodini, and M. Fontanesi, "Comparison between fast-sweep Langmuir probe and triple probe for fluctuations measurements," Review of Scientific Instruments, vol. 72, no. 1, pp. 461464, January 2001.

[120] J. Rodriguez, F. Yousif, E. Fuentes, F. Vazques, and H. Valencia, "Interpretations of Langmuir probe data for positive ion number density of N2O DC plasma discharge," IEEE Transactions on Plasma Science, vol. 42, no. 3, pp. 813-819, March 2014.

[121] A. Rousseau, E. Teboul, and S. Bechu, "Comparison between Langmuir probe and microwave autointergerometry measurements at intermediate pressure in an argon surface wave discharge," Journal of Applied Physics, vol. 98, p. 083306, 2005.

[122] D. N. Ruzic, Electric Probes for Low Temperature Plasmas. The Education Committee of the American Vacuum Society, 1994.

[123] B. Sands, N. Siefert, and B. Ganguly, "Design and measurement considerations of hairpin resonator probes for determining electron number density in collisional plasmas," Plasma Sources Science and Technology, vol. 16, pp. 716-725, 2007.

[124] J. SanMartin and R. Estes, "The orbital-motion-limited regime of cylindrical Langmuir probes," Physics of Plasmas, vol. 6, no. 1, pp. 395405, November 1999.

[125] G. Schulz and S. Brown, "Microwave study of positive ion collection by probes," Physical Review, vol. 98, pp. 1642-1649, 1955. 
[126] C. Shih and E. Levi, "Effect of collisions on cold ion collection by means of Langmuir probes," AIAA Journal, vol. 9, no. 9, pp. 1673-1678, September 1971.

[127] P. Smy, "The use of Langmuir probes in the study of high pressure plasmas," Advances in Physics, vol. 25, no. 5, pp. 517-553, 1976.

[128] Z. Sternovsky and S. Robertson, "Effect of charge exchange ions upon Langmuir probe current," Applied Physics Letters, vol. 81, no. 11, pp. 1961-1963, September 2002.

[129] Z. Sternovsky, S. Robertson, and M. Lampe, "The contribution of charge exchange ions to cylindrical Langmuir probe current," Physics of Plasmas, vol. 10, no. 1, pp. 300-309, January 2003.

[130] Z. Sternovsky, S. Robertson, and M. Lampe, "Ion collection by cylindrical probes in weakly conditional plasmas: Theory and experiment," Jounrnal of Applied Physics, vol. 94, pp. 1374-1381, 2003.

[131] I. Sudit and F. Chen, "RF compensated probes for high-density discharges," Plasma Sources Science and Technology, vol. 3, pp. 162-168, 1994.

[132] I. Sudit and R. Woods, "A study of the accuracy of various Langmuir probe theories," Journal of Applied Physics, vol. 76, no. 8, pp. 4488-4498, October 1994.

[133] J. Swift and M. Schwar, Electrical Probes for Plasma Diagnostics. New York: American Elsevier Publishing Company, 1969.

[134] F. Taccogna, S. Longo, and M. Capitelli, "Ion orbits in a cylindrical Langmuir probe," Physics of Plasmas, vol. 13, p. 043501, 2006.

[135] M. Tichy, M. Sicha, P. David, and T. David, "A collisional model of the positive ion collection by a cylindrical Langmuir probe," Contributions to Plasma Physics, vol. 34, pp. 59-68, 1994.

[136] M. Tichy, P. Kurdrna, J. Behnke, C. Csambal, and S. Klagge, "Langmuir probe diagnostics for medium pressure and magnetised low-temperature plasma," Journal de Physique IV, vol. C4, pp. 397-411, October 1997.

[137] D. Tilley, A. Gallimore, A. Kelly, and R. Jahn, "The adverse effect of perpendicular ion drift flow on cylindrical triple probe electron temperature measurements," Review of Scientific Instruments, vol. 65, no. 3, pp. 678681, March 1994. 
[138] D. Voloshin, A. Kovalev, Y. Mankelevich, O. Proshina, T. Rakhimova, and A. Vasilieva, "Evaluation of plasma density in RF CCP discharges from ion current to Langmuir probe: experiment and numberical simulation," The European Physical Journal D, vol. 69, p. 23, 2015.

[139] J. Waymouth, "Perturbation of a plasma by a probe," Physics of Fluids, vol. 7, no. 11, pp. 1843-1854, November 1964.

[140] J. Waymouth, "Perturbation of electron energy distribution by a probe," Journal of Applied Physics, vol. 37, no. 11, p. 4492, November 1966.

[141] N. Hershkowitz, Plasma Diagnostics, O. Auciello and D. Flamm, Eds. Elsevier, 1989.

[142] R. Huddlestone and S. Leonard, Plasma Diagnostic Techniques, R. Huddlestone and S. Leonard, Eds. Academic Press, 1965.

[143] N. Hershkowitz, "Sheaths: More complicated than you think," Physics of Plasmas, vol. 12, p. 055502, 2005.

[144] T. Sheridan and J. Goree, "Collisional plasma sheath model," Physics of Fluids, vol. 3, pp. 2796-2804, 1991.

[145] I. Hutchinson, Principles of Plasma Diagnostics. Cambridge: Cambridge University Press, 1987.

[146] O. Bilyk, M. Holik, A. Pysanenko, J. Glosik, P. Kudrna, R. Plasil, and M. Tichy, "Langmuir probe diagnostic for measurement of recomination rates of positive ions with electrons in stationary arfterglow system," Vacuum, vol. 76, pp. 457-463, 2004.

[147] J.-S. Chang, G. Ogram, R. Hobson, and S. Teii, "The instantaneous triple probe method for the direct display of plasma parameters in a low density continuum plasma," Journal of Physics D: Applied Physics, vol. 13, pp. 1083-1092, 1980.

[148] Z. Zakrzewski and T. Kopiczynski, "Effect of collisions on positive ion collection by a cylindrical Langmuir probe," Plasma Physics, vol. 16, no. 12, pp. 1195-1198, 1974.

[149] V. Kolobov and V. Godyak, "Nonlocal electron kinetics in collisional gas discharge plasmas," IEEE Transactions on Plasma Science, vol. 23, no. 4, pp. 503-531, August 1995. 
[150] H. Mott-Smith and I. Langmuir, "The theory of collectors in gaseous discharges," Physical Review, vol. 28, pp. 727-763, 1926.

[151] J. Allen, B. Annartone, and U. deAngelis, "On the orbital motion limited theory for a small body at floating potential in a Maxwellian plasma," Journal of Plasma Physics, vol. 63, no. 4, pp. 299-309, 2000.

[152] C. Shih and E. Levi, "Transient performance of negatively biased Langmuir probes," AIAA Journal, vol. 10, no. 1, pp. 104-110, January 1972.

[153] N. Mutuskura, K. Kobayashi, and Y. Machi, "Monitoring of a radiofrequency glow-discharge plasma," Journal of Applied Physics, vol. 66, pp. 4688-4695, 1989.

[154] F. Yousif and A. Mondragon, "An investigation into electron temperature, number density, and optical emission spectroscopy of the dc hollow-cathode plasma discharge in n2o," IEEE Transactions on Plamsa Science, vol. 40, no. 6, pp. 1715-1723, June 2012.

[155] S.-H. Jang, G.-H. Kim, and C.-W. Chung, "In situ method for real time measurement of dielectric film thickness in plasmas," Journal of Applied Physics, vol. 107, no. 2, p. 023303, 2010.

[156] S. Ghosh, K. Barada, P. Chattopadhyay, J. Ghosh, and D. Bora, "Localized electron heating and density peaking in downstream helicon plasma," Plasma Sources Science and Technology, vol. 24, p. 034011, 2015.

[157] F. Chen, "Numerical computations for ion probe characteristics in a collisionless plasma," Journal of Nuclear Energy Part C, vol. 7, no. 1, pp. $47-67,1965$.

[158] F. Chen, "Lecture notes on langmuir probe diagnostics," in Mini-Course on Plasma Diagnostics, IEEE-ICOPS Meeting, 2003.

[159] J. Goree, "Ion trapping by a charged dust grain in a plasma," Physical Review Letters, vol. 69, no. 2, pp. 277-280, July 1992.

[160] J. Allen, R. Boyd, and P. Reynolds, "The collection of positive ions by a probe immersed in a plasma," Proceedings of the Physical Society, vol. 70, no. 3, pp. 297-304, March 1957.

[161] I. Bernstein and I. Rabinowitz, "Theory of electrostatic probes in lowdensity plasma," Physics of Fluids, vol. 2, no. 2, pp. 112-121, March 1959. 
[162] L. Talbot and Y. Chou, Rarefied Gas Dynamics. Academic Press, 1966.

[163] D. Bohm, The Characteristic of Electrical Discharges in Magnetic Fields, A. Guthrie and R. Wakerling, Eds. New York: McGraw-Hill, 1949.

[164] V. Godyak, Plasma-Surface Interaction and Processing of Materials, ser. NATO ASI Series E, O. Auciello, A. Gramsmarti, J. Vallesbarca, and D. Flamm, Eds. Dordecht: Kluwer Academic, 1990, vol. 176.

[165] A. Salabas, L. Marques, J. Jolly, G. Gousset, and L. Alves, "Systematic characterization of low-pressure capacitively coupled hydrogen discharges," Journal of Applied Physics, vol. 95, pp. 4605-4620, 2004.

[166] A. Salabas, G. Gousset, and L. Alves, "Two-dimensional fluid modelling of charged particle transport in radio-frequency capacitively coupled discharges," Plasma Sources Science and Technology, vol. 11, pp. 448-465, 2002 .

[167] L. Marques, J. Jolly, and L. Alves, "Capacitively coupled radio-frequency hydrogen discharges: the role of kinetics," Journal of Applied Physics, vol. 102, p. 063305, 2007.

[168] L. St-Onge and M. Moisan, "Hydrogen atom yield in RF and microwave hydrogen discharges," Plasma Chemsitry and Plasma Processing, vol. 14, pp. 87-116, 1994.

[169] C.-K. Chen, T.-C. Wei, L.R. Collins, and J. Phillips, "Modelling the discharge region of a microwave generated hydrogen plasma," Journal of Physics D: Applied Physics, vol. 32, pp. 688-698, 1999.

[170] E. Hollmann and A. Pigarov, "Measurement and modeling of molecular ion concentrations in a hydrogen reflex-arc discharge," Physics of Plamsas, vol. 9, no. 10, pp. 4330-4339, October 2002.

[171] S. Lakshmanan and W. Gill, "A novel model of hydrogen plasma assisted chemical vapor deposition of copper," Thin Solid Films, vol. 338, pp. 24-39, 1999.

[172] I. Mendez, F. Gordillo-Vazquez, V. Herrero, and I. Tanarro, "Atom and ion chemistry in low pressure hydrogen DC plasmas," Journal of Physical Chemistry, vol. 110, pp. 6060-6066, 2006. 
[173] S. Nunomura and M. Kondo, "Characterization of high-pressure capacitively coupled hydrogen plasmas," Journal of Applied Physics, vol. 102, p. 093306, 2007.

[174] V. Shakhatov and Y. Lebedev, "Collisional-radiative model of hydrogen low-temperature plasma: processes and cross sections of electron-molecule collisions," High Temperature, vol. 49, pp. 257-302, 2011.

[175] B. McCall and T. Oka, "H3+ - an ion with many talents," Science, vol. 287, pp. 1941-1942, March 2000.

[176] I. Tanarro, V. Herrero, E. Carrasco, and M. Jimenez-Redondo, "Cold plasma chemistry and diagnostics," Vacuum, vol. 85, pp. 1120-1124, 2011.

[177] J.-P. Boeuf, "Numerical model of rf glow discharges," Physical Review A, vol. 36, no. 6, pp. 2782-2792, September 1987.

[178] E. A. Baxter, "Final report on the piezoelectric transformer plasma source to los alamos national laboratory," March 2014.

[179] A. Dunaevsky, K. Chirko, Y. Krasik, J. Felsteiner, and V. Bershtam, "Spectroscopy of a ferroelectric plasma cathode," Journal of Applied Physics, vol. 90, no. 8, pp. 4108-4114, October 2001.

[180] T. Sugano, Ed., Applications of Plasma Processes to VLSI Technology. Wiley-Interscience, 1985.

[181] Cryo-Torr High-Vacuum Pump Installation, Operation and Maintenance Instructions, Rev. A ed., Helix Technology Corporation, Nine Hampshire Street Mansfield Massachusetts 02048-9171 U.S.A., April 2001.

[182] J. O’Hanlon, A User's Guide to Vacuum Technology. Wiley, 1980.

[183] COMSOL 3.5a, COMSOL, Burlington, MA, www.comsol.com.

[184] E. Harrison, "Investigation of the perveance and beam proviles of an aperture disk emission system," Journal of Applied Physics, vol. 29, pp. 909-913, June 1958.

[185] S. Klagge and M. Tichy, "A contribution to the assessment of the influence of collisions on the measurements with Langmuir probes in the theick sheath working regime," Czechoslovak Journal of Physics B, vol. 35, no. 9, pp. 988-1006, 1985. 


\section{VITA}

Emily Baxter graduated in December 2008 with Bachelors in Science in Electrical Engineering and Computer Engineering from the University of Missouri. She graduated with a Master of Sceince in Electrical Egineering from the University of Missouri in December 2011. She received her Ph.D. in May 2017. 